University of South Florida

DIGITAL COMMONS

Digital Commons @ University of

@ UNIVERSITY OF SOUTH FLORIDA

South Florida

USF Tampa Graduate Theses and Dissertations

USF Graduate Theses and Dissertations

January 2013

\title{
A Novel Method For Watermarking Sequential Circuits
}

Matthew Lewandowski

University of South Florida, mlewando@mail.usf.edu

Follow this and additional works at: https://digitalcommons.usf.edu/etd

Part of the Computer Engineering Commons

\section{Scholar Commons Citation}

Lewandowski, Matthew, "A Novel Method For Watermarking Sequential Circuits" (2013). USF Tampa Graduate Theses and Dissertations.

https://digitalcommons.usf.edu/etd/4528

This Thesis is brought to you for free and open access by the USF Graduate Theses and Dissertations at Digital Commons @ University of South Florida. It has been accepted for inclusion in USF Tampa Graduate Theses and Dissertations by an authorized administrator of Digital Commons @ University of South Florida. For more information, please contact digitalcommons@usf.edu. 
A Novel Method For Watermarking Sequential Circuits

by

Matthew Lewandowski

A thesis submitted in partial fulfillment

of the requirements for the degree of

Master of Science in Computer Engineering

Department of Computer Science and Engineering

College of Engineering

University of South Florida

Major Professor: Srinivas Katkoori, Ph.D.

Swaroop Ghosh, Ph.D.

Jay Ligatti, Ph.D.

Date of Approval:

March 18, 2013

Keywords: Automata, Encoding, Intellectual Property, Finite State Machine Watermarking, Circuit Watermarking

Copyright (C) 2013, Matthew Lewandowski 


\section{Dedication}

I would like to dedicate this work to my beloved family, parents, and siblings. To my mother and father, Kristy and Michael Lewandowski, I cannot thank you enough for your continued support, both emotionally and physically, throughout my many years of academia, and the many more to come.

I also thank my siblings, Eric, Keven, and Craig for being there for me during my times of need, and for teaching me that regardless of my handicap I can overcome any hurdle when driven by a limitless passion. I am grateful for every lesson you have taught me, every opportunity you have provided me with, and the many years of time you have invested into dealing with me. I can only hope you are as proud of me as I am of you, and I could not have accomplished the things I have today without your continued love and support.

In addition, I dedicate this to Amanda Lewandowski. Without your continued support both as a member of my family and as my physical therapist, I would not have been able to regain the functionality in my hand which allowed me to complete this work. For this time, patience, and care you put forth during my rehabilitative process, I so deeply thank you.

Lastly, I dedicate this to my beloved grandfather and grandmother, Dean and Doris Moon, who have continuously supported me through every life venture and taught me that with the right mind set and attitude, I have the potential to walk among the stars. 


\section{Acknowledgments}

First and foremost, I give acknowledgement with the utmost appreciation and gratitude to Dr. Srinivas Katkoori for providing me with the wonderful opportunity of working on this project. I am grateful to him for letting me be his teaching assistant in the areas of study I so passionately love, which granted me the resources to further my education and opportunities here at the university. I also extend my appreciations and gratitude to Dr. Swaroop Ghosh and Jay Ligatti for serving on my supervisory committee.

I would like to thank Christopher Bell and Matthew Morrison for providing me the opportunity to work alongside them on various hardware security and quantum computing projects. I extend thanks to Richard Meana for his sub-graph matching contributions and involvement with this work during our senior project with Dr. Katkoori. To my lab neighbor, Joseph Botto, I thank you for the grand idea of file hashing and continued technical support.

To my loving friends who were there for me over my academic travels and times of need: Christopher Bell, TracyWolf, Donald Ray, Matthew Morrison, Richard Meana, Nicole Gonzalez, Chris Bringes, Thomas Peterson, Mary Ivory, Cybil Scott, Virginia Maurer, Caitlin Snell, James Adkins, David ODonnell, Roya Kashani, Robert O'Brien, Christo-

pher Denton, Andrew Thomas, Nicholas Carter, Kristen Kluberdanz, Andrew Price, and Benjamin Geiger.

Lastly, I want to acknowledge the wonderful faculty — and staff, current and former, of the University of South Florida Department of Computer Science and Engineering. You have made my academic pursuits of higher knowledge a joyful and pain free experience, and I thank you for that. Students here are truly lucky to have faculty and staff — that are as caring as each and every member proves. 
Table of Contents

List of Tables $\quad$ iii

List of Figures $\quad$ V

Abstract vii

1 Introduction and Background 1

1.1 Thesis Organization 2

2 Modeling Sequential Systems 4

2.1 Finite State Machine Model 4

2.1.1 Asynchronous and Synchronous FSMs 4

2.2 Representations of FSMs 5

2.2.1 State Transition Graphs 5

2.2.2 State Transition Tables 6

2.2.3 Kiss2 6

2.3 FSM Models and Classifications $\quad 7$

2.3.1 Moore Model $\quad 8$

2.3.2 Mealy Model 8

2.3.3 Completely Specified Finite State Machine (CSFSM) 9

2.3.4 Incompletely Specified Finite State Machine (ISCFSM) 10

2.4 State Encoding for Sequential Circuit Optimization 11

$\begin{array}{lll}2.5 & \text { Chapter Summary } & 12\end{array}$

3 Related Work 13

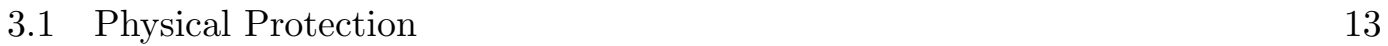

$\begin{array}{lll}3.1 .1 & \text { Integrated Circuit Logos } & 13\end{array}$

3.1.2 Constraint Based Watermarking $\quad 15$

3.2 Hardware Description Language Level Protection 17

$\begin{array}{lll}3.3 & \text { Circuit and Model Level Protection } & 18\end{array}$

$\begin{array}{ll}3.4 & \text { Sequential Circuit Watermarking Techniques } \\ \end{array}$

$\begin{array}{lll}3.4 .1 & \text { State Based Watermarking } & 19\end{array}$

3.4.2 Edge Based Watermarking 20

3.4.3 Input Output Based Watermarking 23

3.5 Motivation for This Work 25

$\begin{array}{lll}3.6 & \text { Chapter Summary } & 29\end{array}$

4 State Encoding Based Watermarking 30

4.1 Note to Reader 30

4.2 Watermarking via State Encoding 31

4.3 Edge Creation Cost 31 
4.4 Watermarking System: Overview 32

4.5 Watermark Construction Phase 33

4.5.1 Bitmap Signature Decomposition 33

4.5.2 File Signature Decomposition $\quad 35$

4.5.3 Hashing Signature Decomposition 37

4.5.4 HSD Watermark Construction: Hash-2-K2 38

4.6 Watermark Embedding Phase 40

4.6.1 Embedding: Complexity 40

4.6.2 Brute Force Embedding Algorithm 41

4.6.3 Greedy Embedding Algorithm 45

4.7 Model Generation and Verification Phase 53

4.8 Watermark Extraction Sequence Generation 58

4.9 On the Tampering Hardness of State Encoding Based Watermarking $\quad 59$

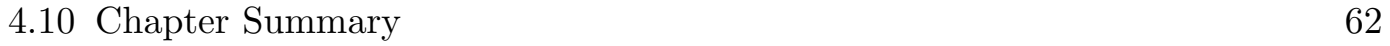

5 Experimental Results 65

$5.1 \quad$ Note to Reader $\quad 65$

5.2 Xilinx Synthesis Options 65

$\begin{array}{ll}5.3 & \text { Benchmark Suite }\end{array}$

$\begin{array}{lll}5.4 & \text { Overhead Calculations } & 67\end{array}$

$\begin{array}{lll}5.4 .1 & \text { User Encoding } & 68\end{array}$

$\begin{array}{lll}\text { 5.4.2 Gray Encoding } & 69\end{array}$

$\begin{array}{lll}\text { 5.4.3 Johnson Encoding } & 70\end{array}$

5.4.4 One-hot Encoding 71

$\begin{array}{lll}\text { 5.4.5 Sequential Encoding } & 73\end{array}$

$\begin{array}{ll}\text { 5.4.6 Speed1 Encoding } & 74\end{array}$

$\begin{array}{lll}5.5 & \text { Discussion of Results } & 75\end{array}$

$\begin{array}{lll}\text { 5.5.1 Synthesis Discrepancies } & 75\end{array}$

5.5.2 Synthesis Results $\quad 75$

6 Future Work $\quad 78$

6.1 Sequential Circuit Logic Synthesis: k3 78

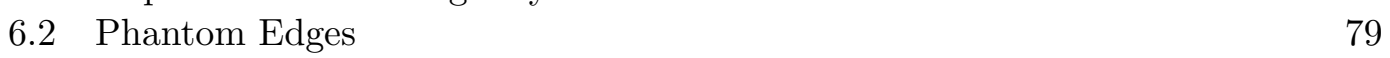

6.2.1 Cost of a Phantom Edge $\quad 80$

$\begin{array}{lll}6.3 & \text { Watermark Extraction } & 80\end{array}$

6.4 Metric Stacking 81

7 Conclusions $\quad 82$

$\begin{array}{ll}\text { References } & 83\end{array}$

$\begin{array}{lll}\text { Appendix A Glossary } & 91\end{array}$

$\begin{array}{lll}\text { Appendix B Permission of Use } & 97\end{array}$

About The Author $\quad$ End Page 
List of Tables

$\begin{array}{llr}\text { Table } 1 & \text { Sample STT representation } & 6\end{array}$

Table 2 Metrics affected by state encoding 11

Table 3 Summary of protection techniques 26

Table 4 Summary of sequential protection techniques 28

Table $5 \quad$ Xilinx synthesis results for dummy edges in Fig. 14 FSM 32

Table 6 Sample file under FSD 35

Table $7 \quad$ Brute force mapping combinations 44

Table 8 Brute force cost mapping combinations 44

$\begin{array}{lll}\text { Table } 9 & \text { Original and watermark FSM node degree values } & 50\end{array}$

Table 10 Example step-by-step greedy algorithm $\quad 52$

Table 11 Embedding results, watermark (Fig. 19) has 15 states and 32 edges 52

Table 12 Time for HPC preimage attacks $\quad 61$

Table 13 Time for HPC collision attacks $\quad 62$

Table 14 Summary of proposed watermark construction phase methods $\quad 62$

Table 15 Summary of proposed watermark embedding phase methods 63

Table 16 Summary of proposed model generation and verification methods $\quad 64$

Table 17 Summary of security run-time analysis 64

Table 18 Xilinx XST optimization options $\quad 65$

Table 19 Top ten largest IWLS'93 Kiss2 files 67

$\begin{array}{lll}\text { Table } 20 & \text { Encoding schemes used } & 68\end{array}$

Table 21 Xilinx synthesis results for User \& User encoded FSMs 69

Table 22 2-bit Gray encoding \& Hamming distance 70

Table 23 Xilinx synthesis results for Gray \& User encoded FSMs 70

$\begin{array}{lll}\text { Table } 24 & \text { Johnson encoding } & 71\end{array}$ 
Table 25 Xilinx synthesis results for Johnson \& User encoded FSMs

Table 26 One-hot encoding

Table 27 Xilinx synthesis results for One-hot \& User encoded FSMs

Table 28 Sequential encoding

Table 29 Xilinx synthesis results for Sequential \& User encoded FSMs

Table 30 Speed1 encoding

Table 31 Xilinx synthesis results for Speed1 \& User encoded FSMs

Table 32 Xilinx synthesis discrepancies for User \& Sequential encoded FSMs

Table 33 Summary of Xilinx synthesis results 76

Table 34 Summary of performance 77

Table 35 k3 overview 78

Table 36 Xilinx synthesis results of phantom edge FSMs

80 


\section{List of Figures}

$\begin{array}{lll}\text { Figure } 1 & \text { STG representation of an example FSM } & 6\end{array}$

$\begin{array}{lll}\text { Figure } 2 & \text { Kiss2 representation of an example FSM } & 7\end{array}$

$\begin{array}{llr}\text { Figure } 3 & \text { Moore model FSM example } & 8\end{array}$

$\begin{array}{lll}\text { Figure } 4 & \text { Mealy model FSM example } & 9\end{array}$

Figure 5 An example of a completely specified FSM (CSFSM) 9

Figure 6 An example of an incompletely specified FSM (ICSFSM) 10

Figure $7 \quad$ Sample IC logo implemented in AMI C5N that passed DRC check $\quad 15$

$\begin{array}{lll}\text { Figure } 8 & \text { Example of hierarchical watermarking } & 16\end{array}$

$\begin{array}{lll}\text { Figure } 9 & \text { Lion FSM watermarked by HARPOON method [1] }\end{array}$

Figure 10 Lion FSM utilizing state based watermarking 20

$\begin{array}{lll}\text { Figure } 11 & \text { FSM utilizing edge based watermarking } & 22\end{array}$

Figure 12 Sample I/O signature 23

Figure 13 FSM utilizing passive I/O based watermarking 25

Figure 14 Watermarking edge creation method an illustrative watermarked FSM 31

Figure 15 High level overview of watermarking system flow 33

Figure 16 Methods of watermark construction 33

Figure 17 Sample bitmap signature 34

$\begin{array}{lll}\text { Figure } 18 & \text { Example of FSD } & 36\end{array}$

$\begin{array}{lll}\text { Figure } 19 & \text { Sample HSD signature prior to hashing } & 37\end{array}$

Figure 20 RIPEMD-160 Pajek netlist / STG using Gephi 38

Figure 21 Hash-2-Kiss2 watermark construction algorithm 39

Figure 22 Proposed brute force watermark embedding algorithm 42

Figure 23 Brute force cost calculation algorithm 43

Figure 24 Original and watermark FSMs for brute force embedding example 43 
Figure 25 Original, watermark, and watermarked FSMs for brute force embedding 45

$\begin{array}{lll}\text { Figure } 26 & \text { Greedy heuristic } & 46\end{array}$

Figure 27 Algorithm for FindMaxDegreeNode function 48

Figure 28 Algorithm for FindMinCostNode function 49

Figure 29 Algorithm for Neighbors function $\quad 50$

$\begin{array}{lll}\text { Figure } 30 & \text { Algorithm for SortDescend function } & 50\end{array}$

Figure 31 Original and watermark FSMs for greedy embedding example 51

Figure 32 Original, watermark, and watermarked FSMs for greedy embedding $\quad 51$

Figure 33 Flow diagram for the custom tool "k2vhdl" 54

Figure 34 Algorithm for VertexCover $\quad 54$

Figure 35 Sample VHDL signal generation for Lion.kiss2 55

Figure 36 Sample VHDL state controller for Lion.kiss2 56

$\begin{array}{lll}\text { Figure } 37 & \text { Sample VHDL state machine for Lion.kiss2 } & 57\end{array}$

Figure 38 Xilinx simulation state encoding trace $\quad 59$

Figure 39 Sample VHDL synthesis options generation for Lion.kiss2 66

$\begin{array}{lll}\text { Figure } 40 & \text { FSM with phantom edges } & 79\end{array}$ 


\begin{abstract}
We present an Intellectual Property (IP) protection technique for sequential circuits driven by embedding a decomposed signature into a Finite State Machine (FSM) through the manipulation of the arbitrary state encoding of the unprotected FSM. This technique is composed of three steps: (a) transforming the signature into a watermark graph, (b) embedding watermark graphs into the original FSM's State Transition Graph (STG) and (c) generating models for verification and extraction. In the watermark construction process watermark graphs are generated from signatures. The proposed methods for watermark construction are: (1) Bitmap Signature Decomposition (BSD), (2) File Signature Decomposition (FSD), and (3) Hashing Signature Decomposition (HSD). The HSD method is shown to be advantageous for all signatures while providing sparse watermark FSMs with complexity $\mathcal{O}\left(n^{2}\right)$. The embedding process is related to the sub-graph matching problem. Due to the computational complexity of the matching problem, attempts to reverse engineer or remove the constructed watermark from the protected FSM, with only finite resources and time, are shown to be infeasible. The proposed embedding solutions are: (1) Brute Force and (2) Greedy Heuristic. The greedy heuristic has a computational complexity of $\mathcal{O}(n \log n)$, where $n$ is the number of states in the watermark graph. The greedy heuristic showed improvements for three of the six encoding schemes used in experimental results. Model generation and verification utilizes design automation techniques for generating multiple representations of the original, watermark, and watermarked FSMs. Analysis of the security provided by this method shows that a variety of attacks on the watermark and system including: (1) data-mining hidden functionality, (2) preimage, (3) secondary preimage, and (4) collision, can be shown to be computationally infeasible. Experimental results for the ten largest IWLS 93 benchmarks that the proposed watermarking technique is a secure, yet flexible, technique for protecting sequential circuit based IP cores.
\end{abstract}




\section{Introduction and Background}

Integrated Circuit (IC) technologies have continued to rapidly evolve in size and complexity since the beginning of their creation. Because of this constant evolution, it has now become a common practice for companies designing Application Specific Integrated Circuits (ASICs) to outsource part of the design process and purchase third party Intellectual Property (IP) cores. These IP cores can be anything from communication to graphics processing units, consisting of combinational or sequential sub-components. By employing this semi-custom design approach, design houses can reduce costs that would typically be incurred from a full-custom design approach. It also enables designers to reduce time-tomarket expectations. Under this business model, the design house will have to pay royalties on every unit sold to the IP owner. It typically only has rights to the IP core for a limited amount of time or design fabrication runs.

One might ponder what is to happen to the IP core that the design house physically has once the licensing period has ended. This is where the need for further IP protection comes into the scenario. There is currently no effective way to stop a company from further utilizing the IP core outside of the contracted licensing period and refrain from rewarding appropriate royalties for the further use of the design. Thus, the IP owner needs another line of protection in the ability to thwart these potentially fraudulent actions. One common way for IP owners to achieve this extra line of protection is through the utilization of digital watermarking techniques. This enables an IP owner to embed a digital watermark for the purpose of ownership verification in the event of litigation. The embedded watermark signature can be any format of digital media ranging from images, audio, text files, and even short length videos.

The process of watermarking electronic art consisted of the IP owners embedding a signature, or watermark, directly into the image in a location that was only known by the owner. This would later enable the owner a method of proving that the work, or 
design, was his or hers if the ownership of the work ever came into question. This was known to be the first "Electronic Water Mark" [2]. Due to technological advancements of networks and electronic libraries [3] since this concept first appeared, this technique would be expanded to encapsulate typography [4]. This would allow publishers, or others sharing typographical material, a method for proving that a given document was their IP. The concept of watermarking was extended to typography through seemingly invisible typographical manipulations, which included the shifting of a single word a mere millimeter to the original document.

Although digital watermarking of IP by included corporate logos in physical layouts is a common today - it should be noted that they can be easily removed by etching

process. As technology complexities would continue to advance over time so too would the spectrum of watermarking techniques and applications for which it could be used. It was applied to ICs first by Charbon [5] through a hierarchical method, and later extended to Field Programmable Gate Array (FPGA) technologies [6] as well as physical design methodologies [7]. Electronic libraries report the earliest extension of this concept to sequential systems was by Olivera [8] where through the implementation of additional Finite Automata and the employment of a secret input sequence to traverse into the watermark, one could successfully embed a digital watermark into a sequential circuit.

This work presents a novel watermarking technique that can be used with sequential systems, and their realized circuitry, by exploiting an inherent characteristic of these machines that was previously unexplored. Given an original model of a sequential system, and the watermark signature, we determine the appropriate matching set which will minimize overhead and properly embed the watermark signature into the original sequential system.

\subsection{Thesis Organization}

In Chapter 2, we present an in depth background on sequential systems. This includes various types of FSM models and classifications, and the different methods for representing these models. In Chapter 3 we will review related work pertaining to physical, Hardware Description Language (HDL), circuit \& model level protection schemes. Additionally, we provide detailed reviews and illustrations of the different techniques for 
watermarking sequential circuits and discuss the motivation which led to this proposed method.

In Chapter 4, we present the proposed method of watermarking. This chapter will cover in depth the technical details of the proposed method. This includes the proposed BSD, FSD, and HSD signature construction methods in sections 4.5.1 through 4.5.3. The complexities of the embedding phase and the proposed Brute Force and Greedy solutions are presented in sections 4.6.1 through 4.6.3. We present the custom "Hash-2-Kiss2," "k2vhdl," and "k2net" tools for automation of model generation and present techniques for verification and watermark extraction. Additionally we present an analysis of the security of the proposed watermarking technique, showing that attacks of reverse engineering and claiming false ownership are computationally infeasible. In Chapter 5 we describe the experimental setup, report the experimental results, and discuss advantages and disadvantages. In Section 5.3 we cover the benchmark suite used and the pre-synthesis experimental watermarking results. In Section 5.2 we cover the synthesis options applied to for the original and watermarked FSM sets. In Section 5.4 we report the synthesis results and overhead calculations for area and frequency for six different encoding schemes. In Chapters 6 and 7 we will outline directions for future work and draw conclusions, respectively. 


\section{Modeling Sequential Systems}

When designing computer hardware based systems the internal subsystems can fall into one of two categories namely, combinational and sequential. Sequential systems differ from combination systems, in that, for a sequential system the history of input sequence may affect the output of the system, while in a combinational system the outputs are only based on the current input combination [9]. In this section, we focus on providing a detailed description and background on using Finite Automata to model sequential systems and components. More specifically this section will focus on the Finite State Machine (FSM) model and representations.

\subsection{Finite State Machine Model}

As previously mentioned sequential systems are those containing memory storage elements which can cause the system to be affected by the previous inputs to the sequential system. An FSM model is an abstract model which can formally describe the behavior of a sequential system, and is a 6 -tuple [10], $F S M<S, I, O, F, H, S_{0}>$. Each parameter of an FSM can be defined as follows: $(S)$ a set of states $\left\{S_{0}, \cdots, S_{k}\right\} ;(I)$ a set inputs $\left\{I_{0}, \cdots, I_{y}\right\} ;(O)$ a set of outputs $\left\{O_{0}, \cdots, O_{x}\right\} ;(F)$ is a set of transitions that represent current states and inputs mapped to next states; $(H)$ is a set of either inputs and states mapped to outputs $S \times I \rightarrow O$ or states mapped to outputs $S \rightarrow O$; and $\left(S_{0}\right)$ is the starting state of the system. FSM models allow greater flexibility in design and enables design automation tools to synthesize a minimal implementation.

\subsubsection{Asynchronous and Synchronous FSMs}

The Input/Output (I/O) behavior of an FSM can fall into two classifications namely, asynchronous or synchronous. Asynchronous systems are those which operate independently of clocking signals. This means that the manner in which the system will update the output 
is based on its arrival to the next state on a transition. The way these systems handle input combinations is also constrained to a mutually exclusive manner, such that, if the current input is " 00 " the next valid input is " 01 " and the input " 11 " is not allowed. This is due to the fact that these systems again operate independently of a clock signal, thus, by allowing more than one input bit to change at once gives rise to the potential for race conditions. This simply means that when exactly two inputs change at once, the signal which propagates quickest will cause change first, i.e., in a input change from " 00 " to " 11 " possible state transitions are also those associated with "10" and "01."

Synchronous systems operate with a reference to a clock signal. This means that any and all system transitions, and output changes, are performed with respect to some property of a clock signal. This property can be either a rising or falling edge of the signal. Synchronous machines allow for greater design flexibility because of the ability for non mutually exclusive bit changes to occur. Thus it allows outputs and system transitions to occur on rising or falling clock edges, removing the potential for race conditions.

\subsection{Representations of FSMs}

There are several ways for representing FSM models for example, graphically, or in tabular format. The remainder of this section will describe popular representation methods for FSMs.

\subsubsection{State Transition Graphs}

The graphical method of representation, the State Transition Graph (STG), utilizes fundamental graph theory units for representing the formal FSM model. A graph consists of vertices (nodes) and edges (links). FSM states and transitions are mapped to nodes and edges respectively. An example of an FSM model represented in STG format is shown in Fig. 1. In this specific example, transitions are mapped to an associated input value, while states are mapped with an associated output value. 


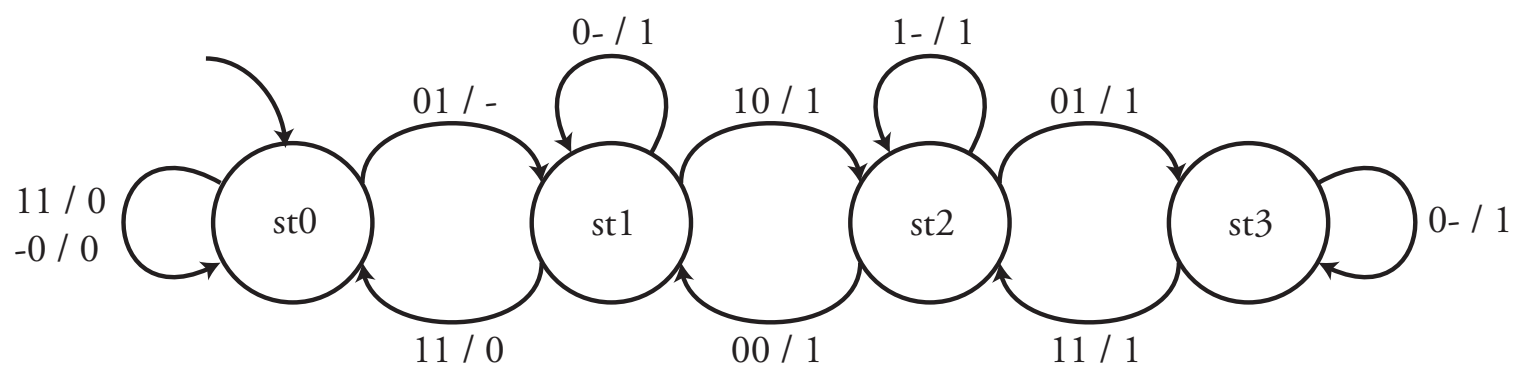

Figure 1: STG representation of an example FSM

\subsubsection{State Transition Tables}

This method of representing an FSM, the State Transition Table (STT), is an equivalent non-graphical, method in tabular format. There are several equivalent methods for representing an FSM through the use of the STT format. However, while the name itself seems to imply only a table of transitions, commonly $[9,11]$ it is a collection of the input, current state, next state, and output, or the combination of the transition and output functions defined by the mathematical model. There are several ways for which an STT can be expressed. To better illustrate this method of FSM representation Table 1 shows a commonly used STT format.

Table 1: Sample STT representation

\begin{tabular}{|c|c|c|c|c|}
\hline \multirow[t]{2}{*}{ Current State (CS) } & \multicolumn{2}{|c|}{ Next State (NS) } & \multicolumn{2}{|c|}{ Output (Z) } \\
\hline & $(\mathrm{x}=0)$ & $(\mathrm{x}=1)$ & $(\mathrm{x}=0)$ & $(\mathrm{x}=1)$ \\
\hline st0 & st1 & st0 & 0 & 0 \\
\hline st1 & st0 & st2 & 0 & 0 \\
\hline st2 & st3 & st1 & 1 & 0 \\
\hline st3 & st3 & st2 & 1 & 0 \\
\hline
\end{tabular}

\subsubsection{Kiss2}

Lastly, with the development of synthesis and optimization tools for sequential systems, the Kiss2 format is an FSM representation and part of the Berkeley Logic Interchange Format (BLIF) circuit description format [12]. The Kiss2 format is an adaptation of both the mathematical model of an FSM and its associated STT representation. It utilizes a human readible syntax to equivalently model an FSM as a 2-tuple, $F S M<D, X>$, 
where $(D)$ is a set of machine descriptors and $(X)$ is the list of information for each row in the corresponding STT representation. Specifically, $(D)$ is the set of following descriptors $\{. i, . o, . p, . s\}$ input length, output length, number of transitions, number of states, respectively. Additionally an optional descriptor .r, or the reset state descriptor, can also be contained in the set $(D)$. The parameter $(X)$ is the set of transitions where each of the transitions follow the format $\{$ input, current state, next state, output $\}$. Figure 2 shows the Kiss2 representation of the STG shown in Fig. 1.

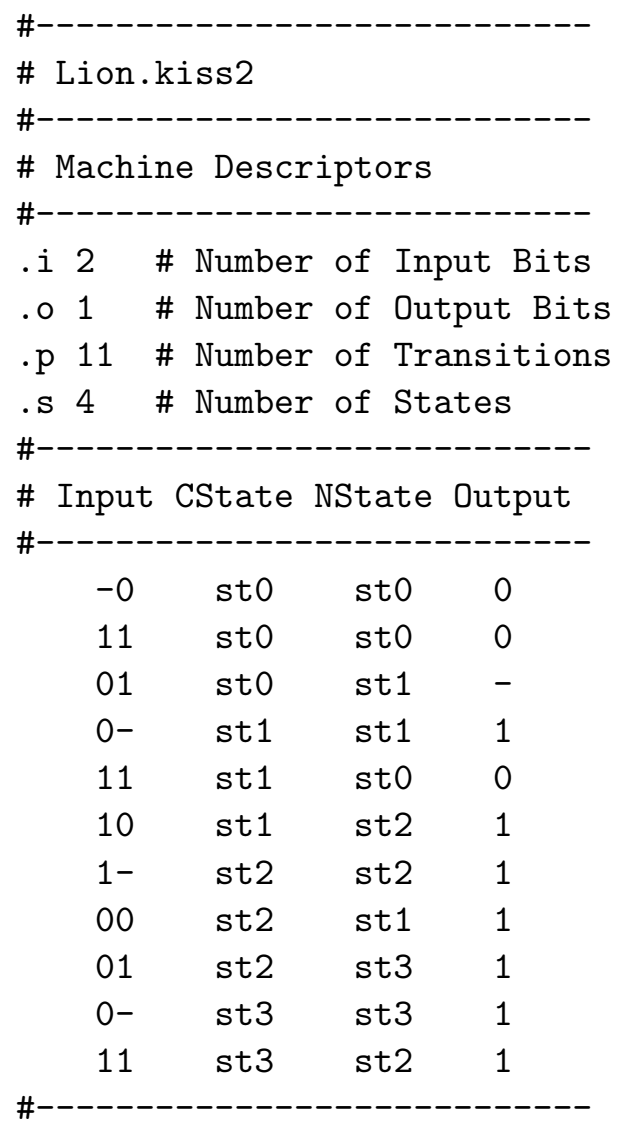

Figure 2: Kiss2 representation of an example FSM

\subsection{FSM Models and Classifications}

Having explored the methods for representing FSMs, in the following sections we explore the two main types of FSM models and the classification groups for which they can 
fall into. First we define two types of models, the Moore and Mealy models. We then define the Completely Specified Finite State Machine (CSFSM) and Incompletely Specified Finite State Machine (ICSFSM).

\subsubsection{Moore Model}

The Moore Model was one of the first methods for graphically modeling sequential systems, and was first proposed in [13]. This model has a specific constraint that pertains to how the output of the system is to be updated. This constraint specifies that the system output is updated after the system has arrived at the next, or destination, state during operation after the edge input condition has been satisfied. An example Moore FSM is shown in Fig. 3. The node labeling format for this modeling is "state encoding value / system output value."

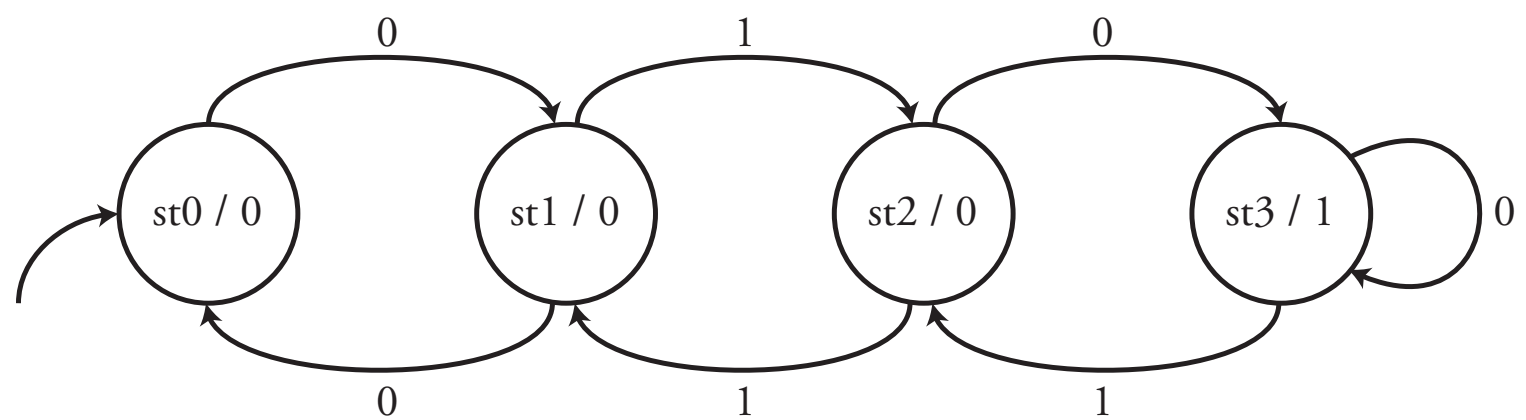

Figure 3: Moore model FSM example

\subsubsection{Mealy Model}

The second method of modeling sequential systems is the Mealy model [14], which operates similar to the Moore Model with the exception of its output modeling constraint. This constraint is that the output is updated on a given edge, or transition, rather than upon the arrival of the systems next state that is dictated by this edge. This constraint allows for systems to drastically reduce the number of states needed to describe system behavior and allows for greater versatility of the system design. An example Mealy FSM is shown in Fig. 4. The edge labeling format for this modeling style is "system input value / system output value." 


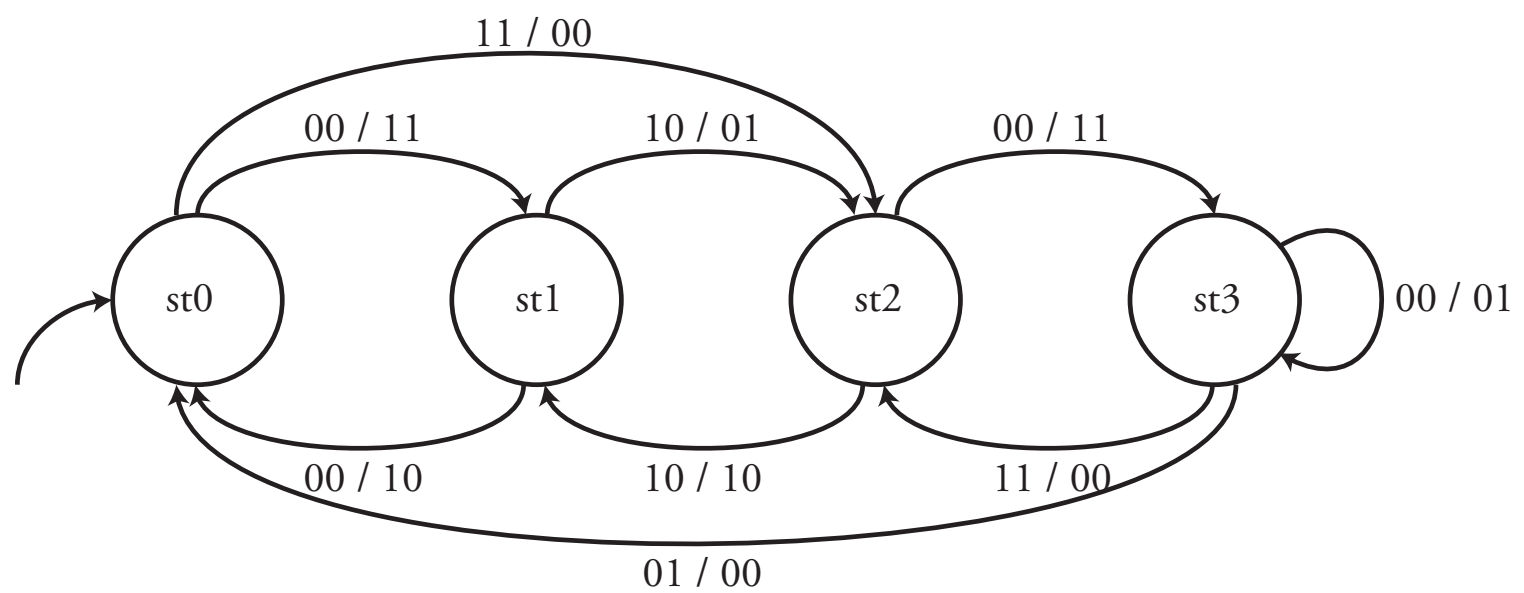

Figure 4: Mealy model FSM example

\subsubsection{Completely Specified Finite State Machine (CSFSM)}

A CSFSM is a Mealy or Moore model which operates under the specific condition that every single possible behavior of the system is explicitly specified by the FSM. The use of the don't care logic conditions in these state machines is prohibited. Figure 5, shows an example of such a machine. It can be seen that all possible behaviors of the system are explicitly stated in the STG. Additionally, because of the conditions in which CSFSMs operate under, they are known to be strictly Deterministic Finite Automata (DFA). This means that there can never be a situation in which one cannot determine the behavior that an FSM will experience, or the resulting location of a transition.

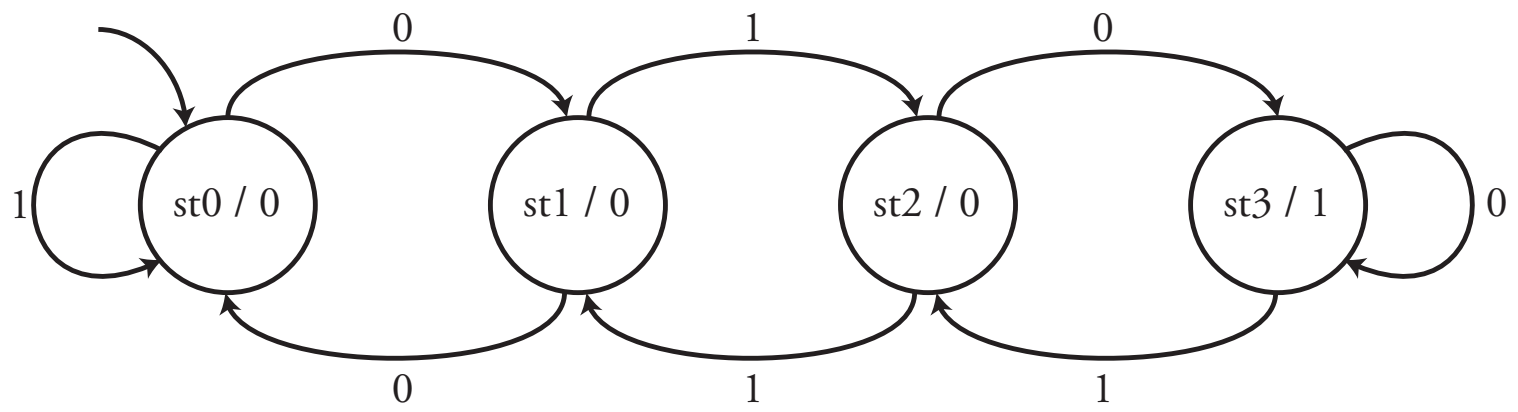

Figure 5: An example of a completely specified FSM (CSFSM) 


\subsubsection{Incompletely Specified Finite State Machine (ISCFSM)}

The ICSFSM is a classification given to a Mealy or Moore model which operates under the specific condition where every single possible behavior of a system is not explicitly specified, or the system employs the use of a multi-value logic system that utilizes the don't care condition. The use of don't care conditions alters the deterministic aspect of the system to potentially non-deterministic behavior. This increases the difficulty of optimization techniques on these machines. This is because a don't care condition, for a single bit, could be either a logical zero or one, and by specifying any don't care condition to either of these values the results produced by optimization techniques may be significantly different. We note that the use of don't care conditions can also apply to the use of states for edge conditions, such that, if a given edge is triggered and the next state is a don't care, then the machine has now become non-deterministic. A machine is labeled as non-deterministic when under any condition there is no way to determine what behavior the machine will exhibit. In such a case, the machines operating under these conditions now becomes classified as Non-Deterministic Finite Automata (NDFA). The FSM shown in Fig. 6 illustrates an ICSFSM. This FSM also exemplifies non-deterministic behavior at "st3."

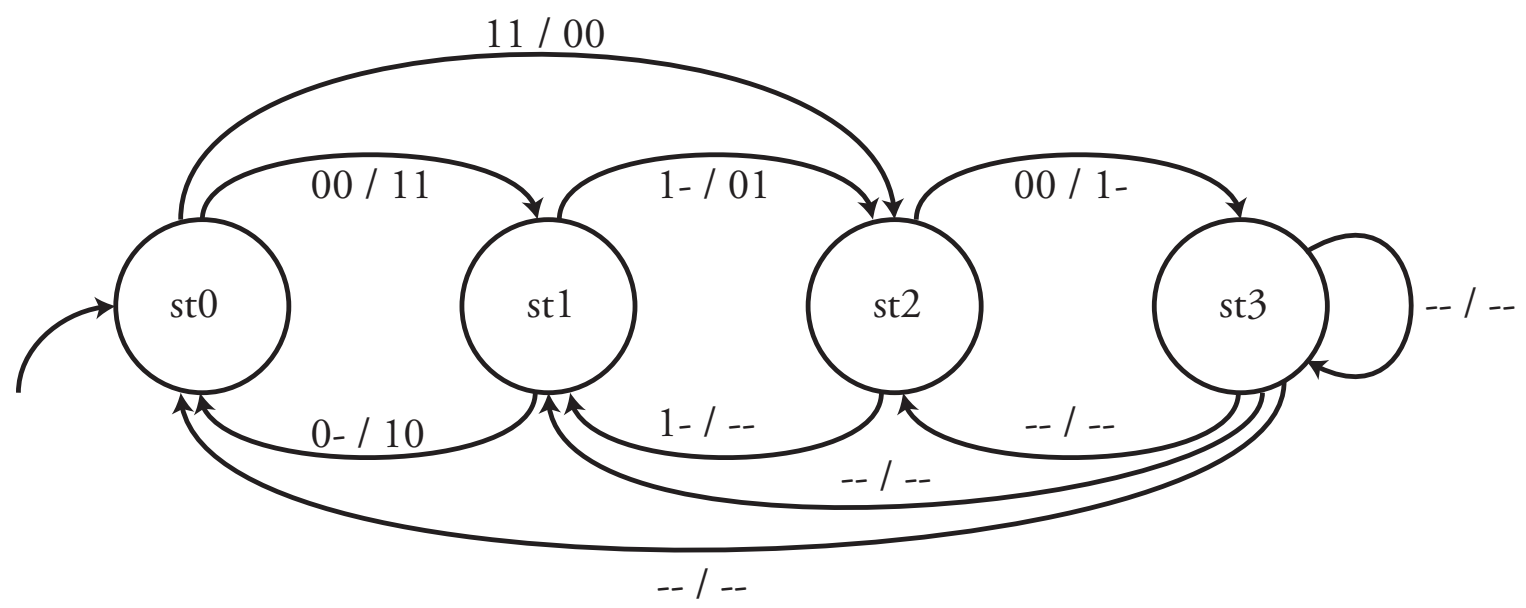

Figure 6: An example of an incompletely specified FSM (ICSFSM) 


\subsection{State Encoding for Sequential Circuit Optimization}

Once an FSM model has been constructed, each of the states in $(S)$ from the formal FSM model assigned a state encoding. State encoding can be viewed as a unique identifier for the state and can either be assigned arbitrarily [15] or intentionally [16-46]. State encoding values can present themselves in either text string or binary bit sets, i.e., either "st0," or "00." Intentionally assigning state encoding values for circuit optimization gives rise to what is commonly known as the State Assignment Problem. This problem can be described as the problem of assigning state codes such that the design metrics of the system, such as, delay, complexity and area, power, and other metrics, can be optimized. Table 2 shows the range of design metrics that can be optimized by the use of suitable state encoding values.

Table 2: Metrics affected by state encoding

\begin{tabular}{|c|}
\hline Related Work: $[\mathbf{1 6}-\mathbf{4 6}]$ \\
\hline Design Metric \\
\hline Area \& Complexity Reduction \\
\hline Built-In Self-Test (BIST) \\
\hline Delay \& Switching Time Reduction \\
\hline Hazard \& Glitch Elimination \\
\hline Low-Power \& Low-Leakage \\
\hline Watermarking \& Security \\
\hline
\end{tabular}

From Table 2, it can be seen that an extensive amount of work in the field of FSM state assignment has been performed. However, while the specific techniques for determining sets of state encoding values that can be assigned for optimization of various design metrics, the underlying concept behind each technique is still the same. It is stated in [11] that a good approach to handling the state assignment problem is by developing a set of guidelines which will reduce the overall complexity of the next state equations and yield a reduced state table. Through the use of the information provided in the STT and the use of Karnaugh Maps [47], or other techniques, the optimal next state equations can be produced for an FSM. This is the general idea behind most methods of state assignment techniques. 


\subsection{Chapter Summary}

There are several ways for representing FSM models which can be achieved through the use of the STG, STT, or Kiss2 method. Each method unique in its own way, the STG is the only visual method for representing this data. However, the non-visual methods are amenable for easy processing by design automation tools. Recapping the types of FSMs there exists both the asynchronous and synchronous versions of these model. In this work all FSM models considered are synchronous machines. Additionally, there are the two main models for FSMs the Moore and Mealy models, where the main difference lies in how the machine updates the output. In the case of Moore model, outputs are updated upon the arrival at the next state. On the other hand, in Mealy model, they are updated during

the transition to the next state. Further the FSMs can be either be completely specified or incompletely specified. 


\section{Related Work}

A significant amount of research has been done in the field of IP Protection and watermarking techniques for ICs at all levels and platforms [1, 5, 7, 8, 45, 46, 48-63]. This includes Soft IP, where designs are easily altered, and Hard IP, where designs are difficult to alter, and range from FPGA devices to IC Layouts. In Sections $3.1-3.3$ we illustrate various methods for IP Protection over several different levels of the design hierarchy. In Section 3.4, we discuss in detail the existing methods of watermarking for sequential systems. The remainder of this chapter, will cover the main areas of IP protection currently available to IP core owners. We will provide an overview and analysis of select methods for the purpose of better illustrating the available protection methods.

\subsection{Physical Protection}

We define physical protection to be the addition of intangible elements, or function-

ality, to pre-fabrication circuit level designs for the purpose of ownership verification. These intangible elements can range from IC company logos to specific place-and-route mapping for standard cells. The remainder of this section will cover several methods of physical layout protection while identifying the pitfalls as well as the advantages of employing them for IP protection. This form of protection is typically applied to Hard IP, or an IP which is in a format difficult to alter.

\subsubsection{Integrated Circuit Logos}

Physical IC layout designers have a long standing tradition of placing logos in the fabrication ready design [64]. However, the evolution of technology, and the intricacy of chip logos has made this a difficult process for fabrication facilities. This is due to two facts, first, the host fabrication facility will ensure that the design intended to be fabricated 
passes all Design Rule Checking (DRC) requirements, and second, that each fabrication ready design follows a set of vendor-independent design rules.

For example, ON-Semiconductor's (AMI) C5 Complementary Metal Oxide Semiconductor (CMOS) fabrication process family contains the N \& F processes [65]. Each of these processes has a set of DRC rules which depend specifically on the feature size and the intended application of the technology, which may be Scalable CMOS (SC), SubMircon (SUBM), or Deep Sub-Micron (DEEP SUBM). In addition to this criteria, each subprocess has an associated, parenthetical, labeling, i.e., AMI_C5N (SCN3ME) and AMI_C5F (SCN3M) processes. These labelings denote a set of descriptors for the technologies physical design process, such that, they are Scalable CMOS (SC) developed entirely in an N substrate $(\mathrm{N})$, while providing use of three metal layers (3M), and even potentially allowing the use of a secondary poly-silicon layer, electrode (E), for poly-capacitors. From these notations it can be seen how quickly this system escalates out of hand even though this is a process family of two. This system becomes even more daunting for fabrication facilities that are required to perform in-depth verification of designs when DRC rules are violated.

Due to the potential design and fabrication issues that these logos can cause, more and more fabrication facilities may not provide a readily available design fabrication layer for indication of on-chip logos for the use of logos. Thus, designs must go through the aforementioned rigorous verification process, in most cases, upon the design failing DRC due to these logos. This is exemplified by fabrication facilities, such as the MOSIS Fabrication Facility, that still heavily discourages the use of logos due to the potential delays they can impose on entire fabrication runs [66]. However, even with heavy discouragement, it is possible to construct an IC Logo that passes DRC rules. Shown in Fig. 7 is a logo that was implemented using the ON-Scemiconductor (AMI) C5N technology, and even though the logo is complex in design it successfully passed DRC checking. More intricate examples can be found at [67], which can be seen as potential fabrication nightmares.

Unfortunately due to the recent increase of companies outsourcing IC fabrication, rather than costly in-house fabrication, smaller non-spacious IC logos have the risk of becoming endangered. This is due to the process of how outsourcing and design fabrication function. Under almost all conditions, the design house will provide the outsourced fab- 


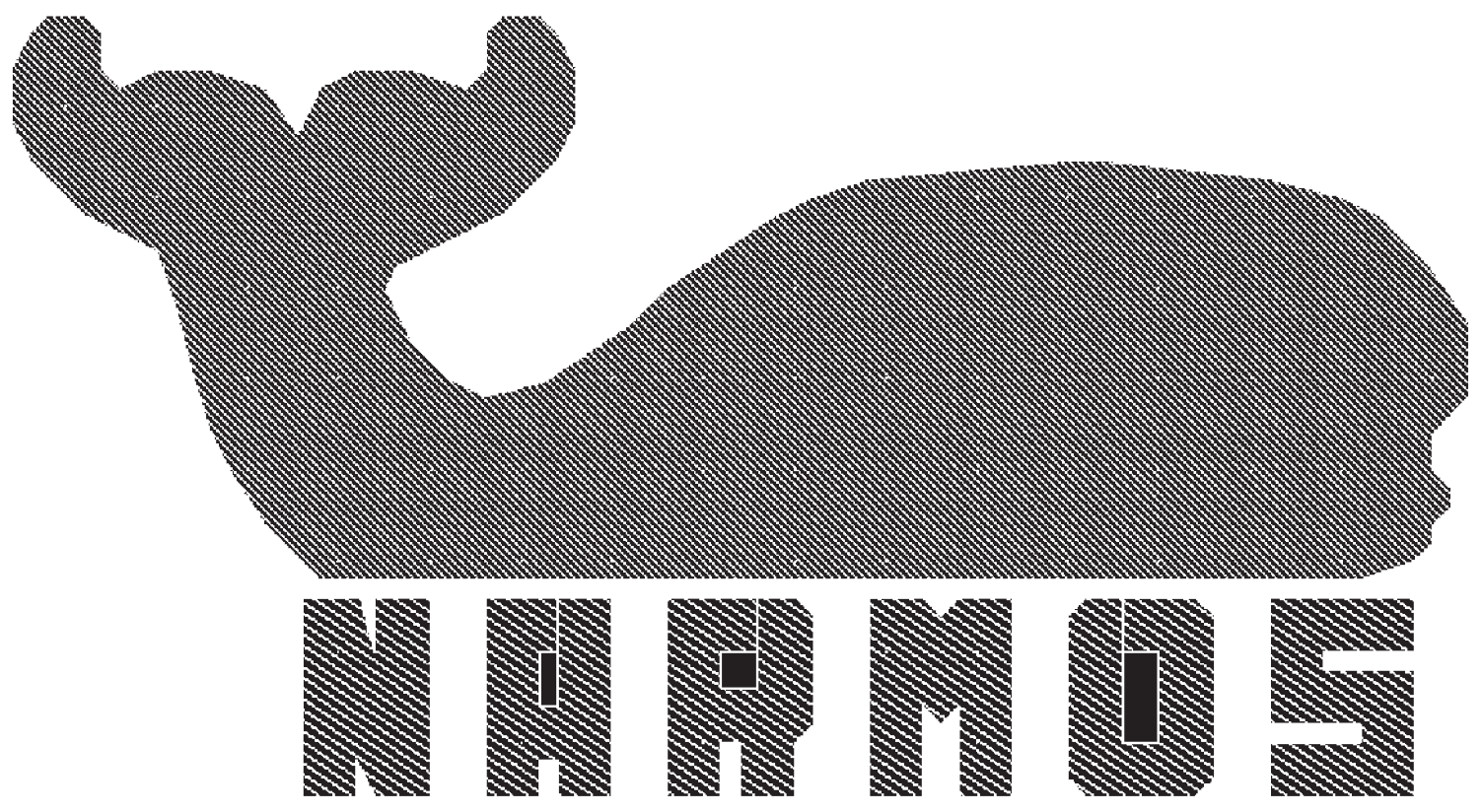

Figure 7: Sample IC logo implemented in AMI C5N that passed DRC check

rication company with a lithographic mask of the final layout. This hand off can lead to potential theft, or counterfeiting, of the IP. This potential misuse can be in the terms of the removal of the company logo, altering, or duplicating, the mask prior to fabrication. However, even if companies continue to use logos, as their viable method for protection, while continuing to outsource fabrication of their ICs, it will most likely come at a cost. This cost is due to the lack of non-invasive methods that allow companies to verify that their logo, still exists after an outsourced fabrication run. This means companies must use high cost destructive methods on post-fabrication ICs to verify that the integrity of the mask and confirm that neither the mask nor design were compromised.

\subsubsection{Constraint Based Watermarking}

Charbon [5] proposed the hierarchical method of watermarking through the implementation of topographical constraints through multiple signatures. This process involves recreating the topological signatures through the floorplan and routing phase of the original design topology. What this simply means is that the signatures are used in generating the specific layout, and placement, of instantiated components based on these topological signatures used as the watermark. This process relies on using a large number of partial 
signatures rather than one signature mapped to the entire topology. This is because the use of a single signature allows for the destruction of the watermark from the addition or deletion of a single component that is to be used in the design layout. The process of signature identification in this technique is shown to be a complicated process. This is due to the use of many partial signatures may have been scrambled throughout the design during synthesis, and to identify the watermark a technique known as genome searching must be used. This searching technique is where a best match the set of partial signatures used in the watermark must be found in the layout. However, while this technique is non-invasive for Soft IP it is also very costly. Additionally post-fabrication watermark verification and extraction will require costly and invasive methods.

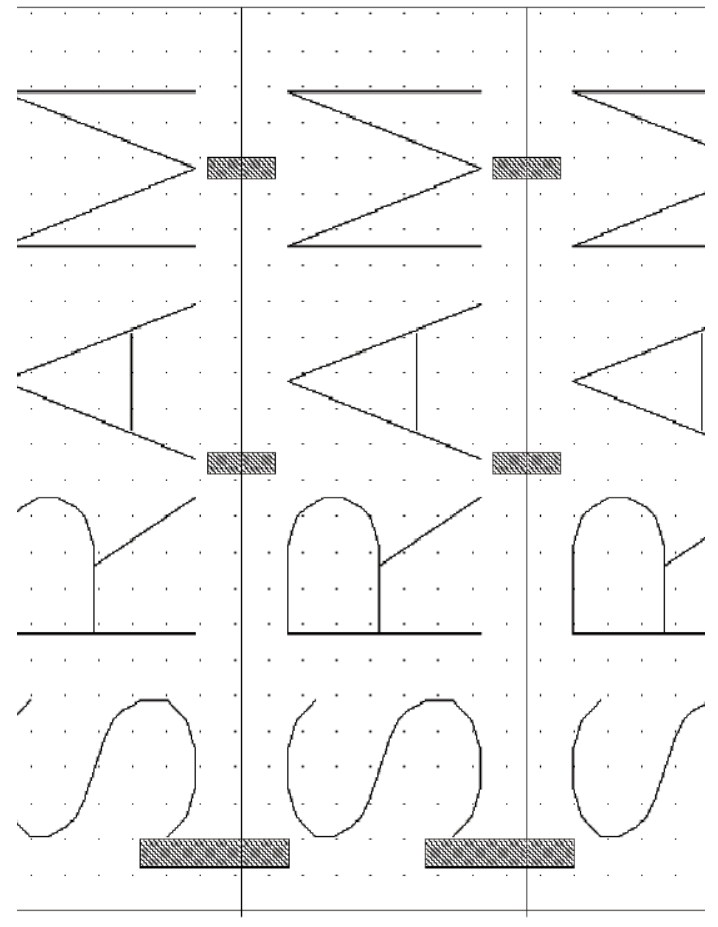

(a) Standard layout

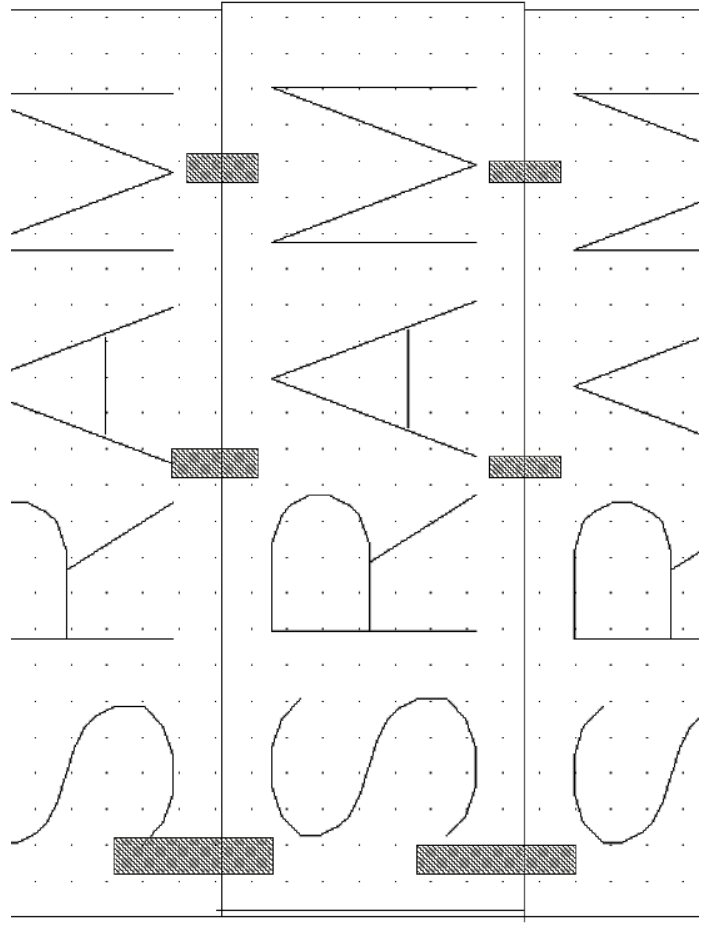

(b) Watermarked layout

Figure 8: Example of hierarchical watermarking

Figure 8 is an illustrative example of this scheme. The general idea is that the topographical watermark is created from layout constraints based on the signature which creates a specific layout from placement and routing that has a low probability of being recreated. In Fig. 8(b) is the watermarked layout, using an arbitrary sequence the single 
Static Random-Access Memory (SRAM) cell is adjusted a mere lambda, or grid unit. However, the probability of recreating this layout is extremely low due to design optimization techniques and algorithms which would create a design that employs a square aspect ratio with minimalistic amounts unused space. Shown in Fig. 8(a) is a similar layout with the exception that it has been designed with no signature constraints that would otherwise adjust the single SRAM cell.

\subsection{Hardware Description Language Level Protection}

The continued development, and advancement, of technologies eventually gave rise to FPGA devices and advanced HDL. The HDL-based netlist could be easily used, and re-used, for a plethora of design systems, and in a medium that was easily transferable and editable, because of this, these formats of IP are known to be Soft IP. Due the development of this new format, IP theft and terms of use violations for contractual agreements of licensed Soft IP cores could occur more easily. As a result and previously mentioned, different methods of protection have been proposed to prevent such practices. However, most of these methods at this level of abstraction are similar in nature, which is by implementing a significant amount of additional functionality. In [1], the authors present a method for obfuscating system level designs which directly relates to the addition of at least one, if not two, extra FSMs in addition to the original FSM being protected. Using a previous example, Fig. 1, we implement this method of protection into the original FSM, which is shown in Fig. 9. For the sake of clarity, the conditions on the edges shown for traversal of the authentication and obfuscation FSM are not labeled.

As illustrated in Fig. 9, it can be seen that there are two additional FSMs needed for the added layer of protection. While the overhead may be small in some cases, the Authentication and Obfuscation FSMs that were added nearly doubled the number of states to be implemented. While this method may be useful in a setting where the desired IP core for protection has been hardened, i.e., a post synthesis FPGA bit file, the alteration of a single transition can render the protection method useless. Alternatively, this protection scheme can be compromised through methods such as that presented in [68], and by using this system, one can decompile bitstream files to low-level netlists for the purpose of easily 


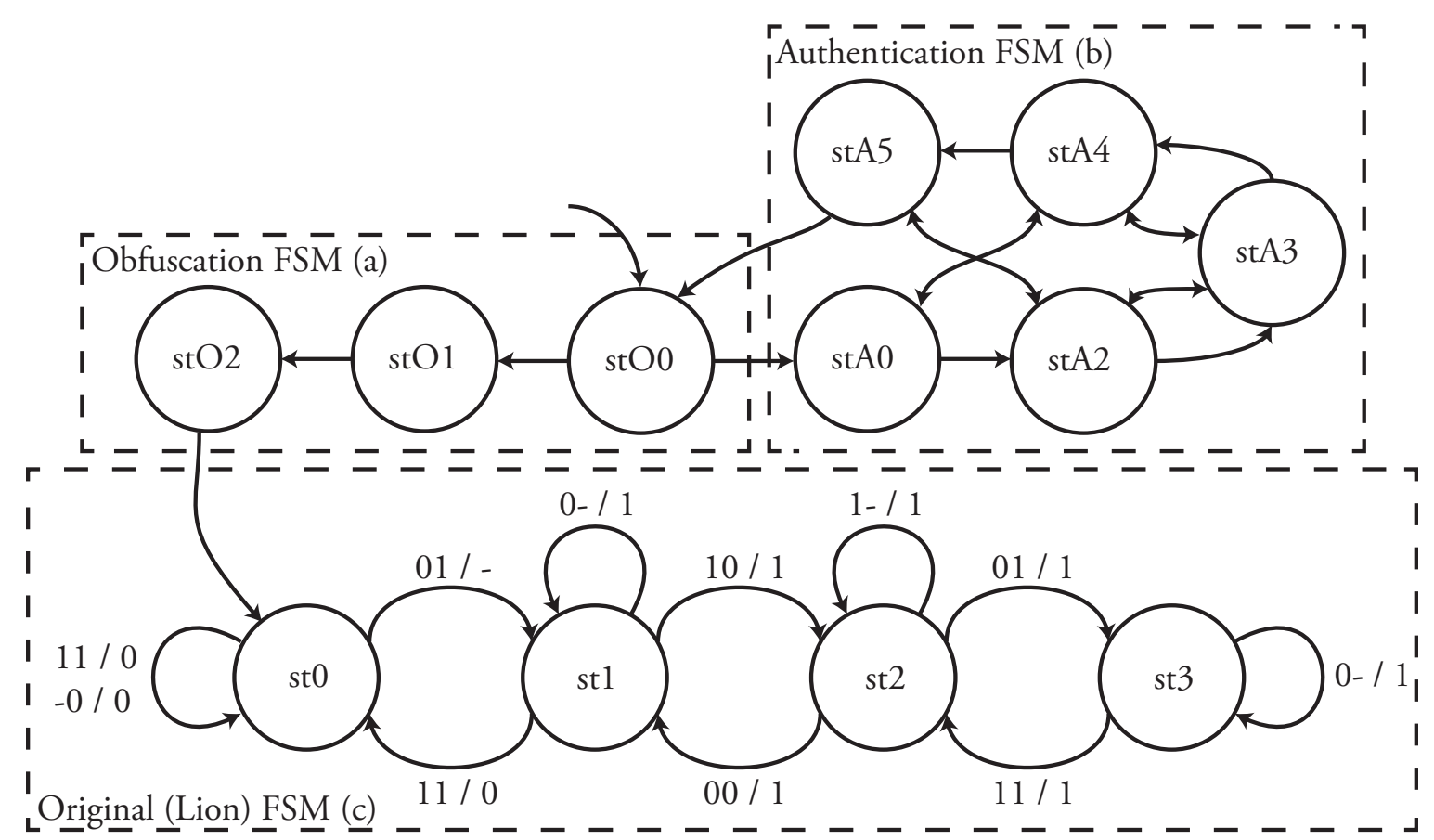

Figure 9: Lion FSM watermarked by HARPOON method [1]

removing and altering such security schemes. However, even though the system presented in [68] may, or may not, be publicly available, other organizations such as [69] are beginning to hold competitive challenges to reverse engineer the bitstream format. It should be known, however, that for significantly complex systems, which utilize the greater portion of design space, will further help prevent the removal of this protection scheme. For relating this type of tampering of the protection scheme we use the idiom of "finding a needle in a hay stack," such that, in a significantly complex system the odds of finding a single transition in the design are significantly low, which further helps to prevent the removal of this protection scheme.

\subsection{Circuit and Model Level Protection}

Circuit level protection is employed through the utilization of the inherent characteristics of a system and the behavior it experiences under given operating conditions. This is commonly referred to as "Glitch Logic," presented in [54], and is geared towards exploiting inherent delay characteristics of logic circuits that would otherwise cause a glitch in the system. Implementation of this method utilizes designs, and practices, that would 
otherwise be considered poor due to the likelihood that a logic implementation will produce faulty, or glitched, output. Utilizing this system allows for signature logic behavior to be produced by an otherwise hidden channel that can be activated under a given set of operating conditions. These operating conditions are constrained by the system but can typically be activated by simply increasing the clock speed to a set frequency outside the operating frequency range of the design. The potential drawback of this system is that a design house is burdened with a daunting task of intentionally implementing, what is seen as poor design, logical hazards into the system while ensuring proper functionality under normal operating conditions.

Model Level protection is similar to the practices detailed by the HARPOON [1] system previously discussed in Section 3.2, and illustrated by Fig. 9. The overall goal of this level of protection is to provide a method that can be utilized at the highest level of abstraction, while offering protection to the IP core through all steps of any further implementation or fabrication processes. With respect to sequential circuits, the current methods of IP protection are detailed in Section 3.4, and are the state, edge, and I/O based schemes of protection.

\subsection{Sequential Circuit Watermarking Techniques}

Several watermarking techniques for sequential systems are currently available to IP core owners. These available watermarking techniques include state based, edge based, and I/O based protection schemes. The remainder of this section will discuss and analyze each of these watermarking techniques.

\subsubsection{State Based Watermarking}

This method of watermarking sequential systems pertains to the direct addition of states into a sequential system. Besides watermarking states, additional states, which act as a key-based system, are inserted to provide security between the watermark and the original FSM. We apply this technique to the FSM shown in Fig. 1 and show the resulting FSM in Fig. 10. We note that the labeling of edges in the watermark key FSM are intentionally left out for the sake of clarity, such that, the key values for entering the watermark are 
arbitrary for the purpose of the illustration of intended functionality. The watermarking method illustrated by Fig. 10 was first introduced by Oliveira in [8]. While this method offers an additional layer of protection, the incurred overhead of this method can escalate rather quickly based on the complexity of the original system with respect to the key and watermark FSMs. However, this can prove to be useful in a significantly large system. On the other hand, when this is applied at the beginning of the modeling level before any form of implementation, synthesis, or fabrication, this method has the potential to be easily defeated by tampering in a design house setting.

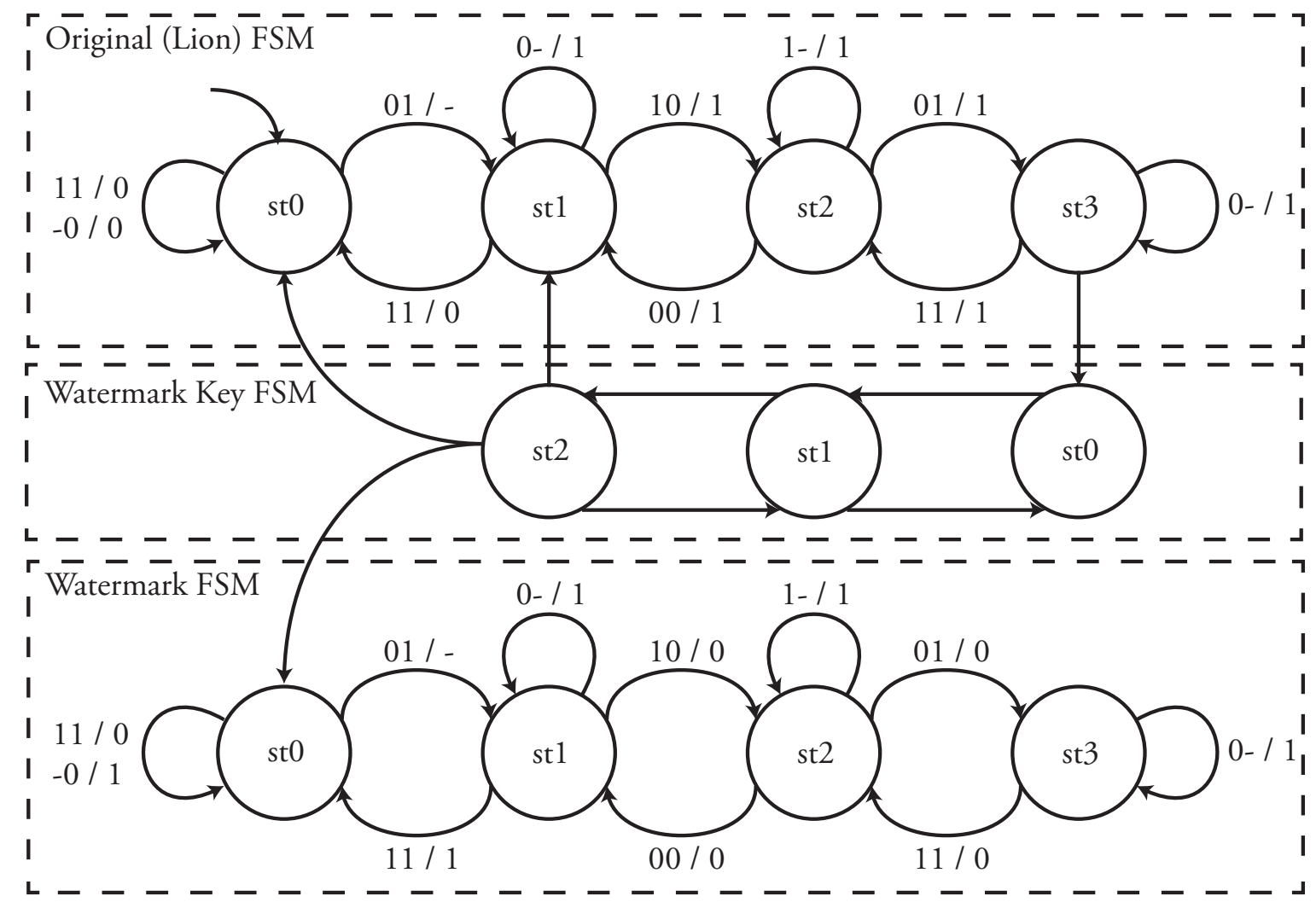

Figure 10: Lion FSM utilizing state based watermarking

\subsubsection{Edge Based Watermarking}

Another technique for the watermarking of sequential systems is through the use of an edge based watermarking scheme. This scheme, presented by Abdel-Hamid et al. [50], breaks the desired signature into a bit length that matches the output length of the FSM intended for watermarking. These output blocks are randomly paired with an input com- 
bination that whose length matches the length of input bits used in the system. Through the process of random start state selection, this technique evaluates its ability to add a signature edge to the selected state. This evaluation is the process of checking the randomly generated input for the signature block against those specified by the selected state. If the signature edge is not being utilized, this edge is simply added. Similarly, if the signature and non-signature edge outputs match, the system utilizes the existing non-signature edge as the signature edge. This is done to reduce the number of edges created by utilizing inherent system functionality to reproduce the desired signature. However, if the required signature edge is being utilized as a non-signature edge and these two edges do not contain equivalent outputs, this method will add an input bit to the system. Upon adding the input bit, the original non-signature transitions are extended, at the Least Significant Bit (LSB) in a Big Endian manner, with zero and the signature edges are extended with one. However, we note that while this work explains the manner in which this technique should operate, it has been published various times [50-52] and each time utilizes the same example, while each illustration used reports conflicting data in the watermarking process. This inconsistency pertains to original edge I/O combinations changing throughout the example, i.e., $\mathrm{I} / \mathrm{O}$ values of single non-signature edge were observed changing as many as three times in a four step example.

Figure 11 illustrates an example of this protection method extended to the Lion FSM, from Fig. 1. We note that this example has been constructed from the interpretation of the watermark insertion algorithm presented in [51]. The desired signature "110" was blocked appropriately to match the length of the system output and paired with random input combinations that match the length of the initial system. In this example specifically the signature was blocked and mapped to "[00/1],[10/1],[01/0]," where the format is "[input/signature]." For the purpose of this example, we start the technique at the starting state and simply work our way to the end. We note that the thicker lines shown in Fig. 11 denote signature edges that were mapped within the system, in addition, edges that were dashed indicate that they were added by the system. Shown by the watermarked FSM in Fig. 11, it can be seen that because the watermark edge input was not being utilized, this signature edge was simply added and the watermarking state now becomes "st1." At "st1" 


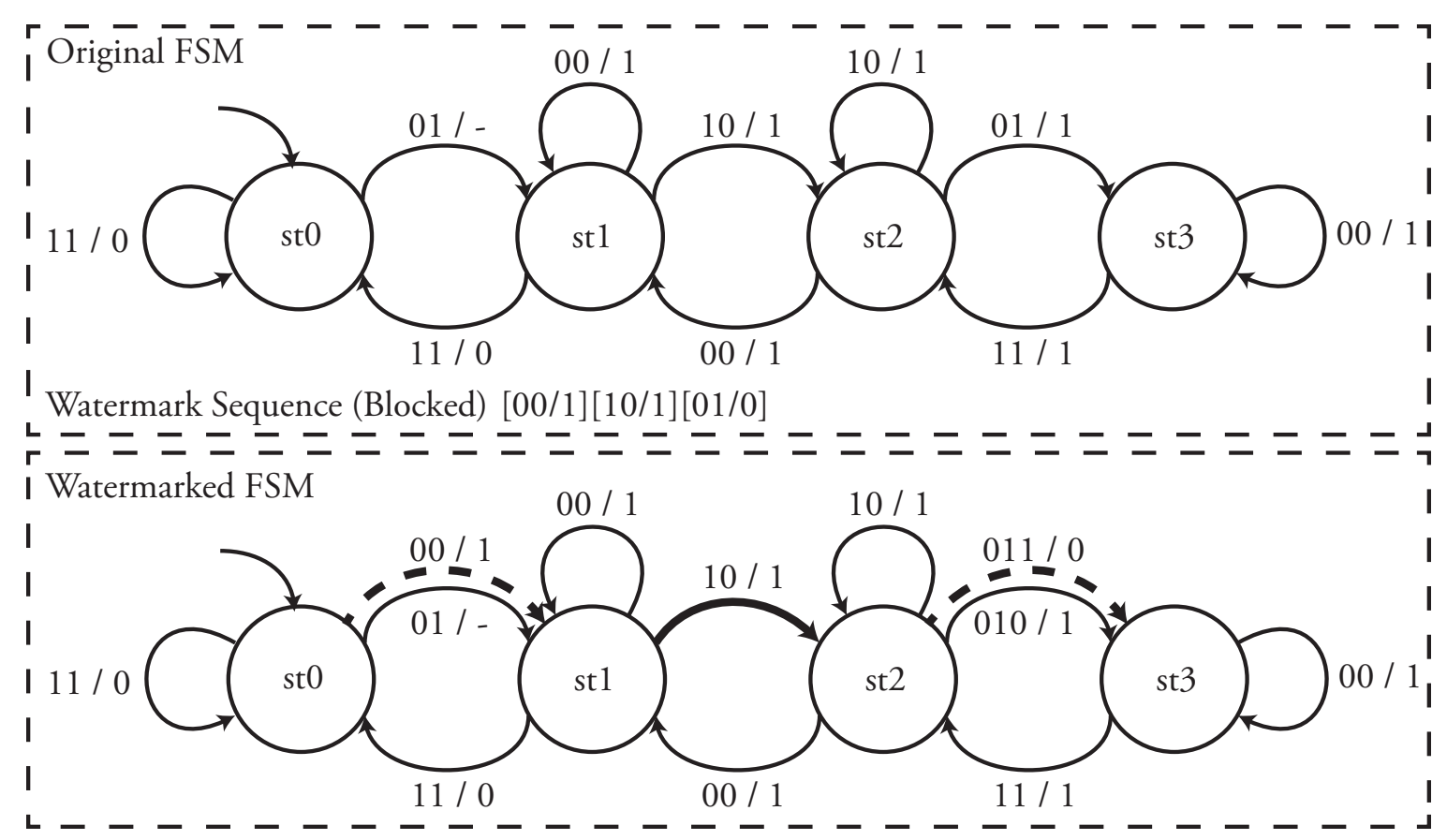

Figure 11: FSM utilizing edge based watermarking

the next edge to be added in the sequence is already utilized, in addition, the signature edge output matches that of the utilized edge and no edges need to be added, allowing us to move to "st2." At "st2" the signature edge and utilized edge output values do not match, and from our interpretation, this is where the LSB of the input string is extended. The extensions that are applied to the signature edge and utilized edge are logical one and logical zero, respectively. While unclear, this method is a low cost alternative to the previous state based watermarking technique from Section 3.4.1.

However, because this watermarking technique directly outputs part of the signature and alters the output of the system this allows for signature edges and utilized edges to be differentiated between. With this in mind, once all of the signature edges have been data mined and extracted this method is rendered useless. This is because now that an attacker has partial blocks of the signature, regardless of what the signature may be, there is no way to prove ownership when both parties claiming ownership know all of the additional functionality. This is based on the fact that once all functionality has been discovered then any desired signature can be created from the known functionality hidden or not. However, if all edges can be completely embedded, i.e., a 100\% match and no created edges, 
then no two sequences are indistinguishable when used as signatures. This holds because there is no secret functionality that would otherwise allow for two signatures to become distinguishable from each other, thus this system requires at least one edge to be added. From this however, additional functionality can be data-mined and easily removed, thus, destroying the signature. Even if signature edges are utilized by non-signature edges along with created edges, if the additionally created edges are removed then there is no way to distinguish from signature and system functionality.

\subsubsection{Input Output Based Watermarking}

The last watermarking technique is that presented in [48], which utilizes I/O sequences native to the original FSM. This technique operates in a manner that utilizes augmenting paths of state transitions to construct a resulting I/O signature, such that, a given input sequence creates an I/O signature from the outputs of the edges traversed. However, this technique achieves this through a passive and active watermarking scheme, each of which are applied to ICSFSMs and CSFSMs respectively. Signatures for this method are based on binary sequences that are generated from the outputs of specific unbound input combinations. A sample signature FSM was constructed using the signature "110," and is shown in Fig. 12.

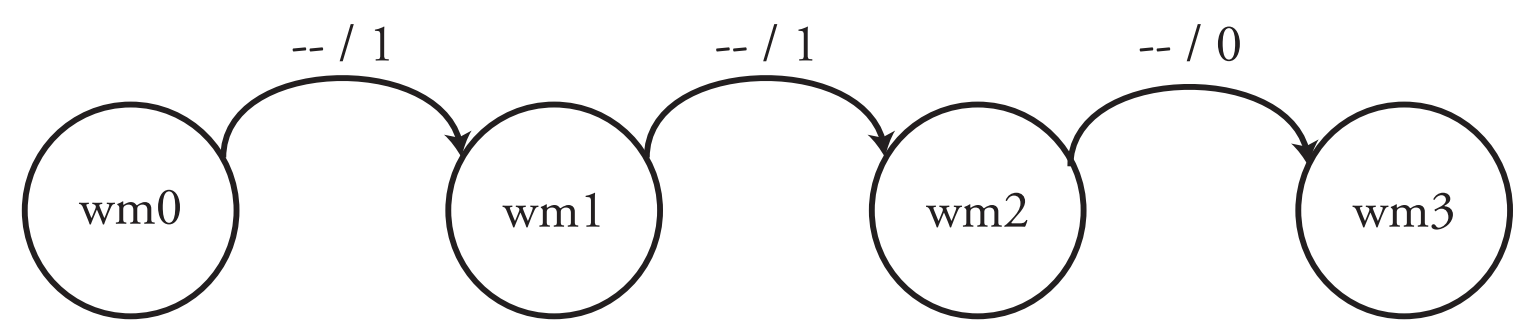

Figure 12: Sample I/O signature

Utilizing such a signature the system algorithmically employs both a passive and active watermarking method. The passive watermarking algorithm is based on utilization of unbound input specifications within the original ICSFSM. These unbound edges are used in an augmenting path algorithm which constructs the input specifications which are bound to the I/O signature. In the event that the original FSM is a CSFSM and the passive scheme 
cannot be utilized, this requires the algorithm to utilize the active algorithm. The active algorithm causes the unbound I/O combinations to become expanded by extending the length of the number of bits used, in this signature example, Fig. 12, the input bit length would need to be extended in the even the initial system was a CSFSM. By extending the number of input bits available the CSFSM, the system is transformed into an ICSFSM. From this bit length extension a number of unbound input combinations are generated, the specific number of total unbound input combinations in the system that will be generated is given by equation 1 .

$$
\begin{gathered}
\Delta \text { Edges }_{\text {unbound }}(\text { System })=\sum_{0}^{\text {states }} 2^{(n+1)}-2^{n}=\sum_{0}^{\text {states }} 2^{n} \\
\Delta \text { Edges }_{\text {unbound }}(\text { State })=2^{(n+1)}-2^{n}=2^{n}
\end{gathered}
$$

From equation (1), it can be seen that by the addition of a single input bit the system now has a number of unbound edges equal to the number of all edges in the original system. Likewise, equation (2) shows that each state has gained an equivalent amount of unbound edges due to the input bit length increase. Doing this will ultimately result in the algorithm creating an augmenting path for the otherwise original CSFSM which it can now map input sequences to the I/O signature. The extraction method for this technique is to simply apply the known sequence that was generated for the I/O mapping and observe the output by applying the sequence, thus making verification of the watermark a simplistic process. Ultimately, this technique is achieved through the addition of edges to generate the I/O watermark. However, it is set apart from previous edge based watermarking techniques due to the embedding technique, which does not contain any previously utilized edges. Figure 13 illustrates the passive method of this watermarking technique.

However, due to the fact that this system employs only edge creation it actually increases the difficulty for proving ownership. This is because once the additional edges have been data-mined, the exact signature can be determined. This is because the system implements a simple path, or simple cycle, when constructing the desired signature. While finding a simple cycle in a graph can be mapped to the Hamiltonian path/cycle problem, 


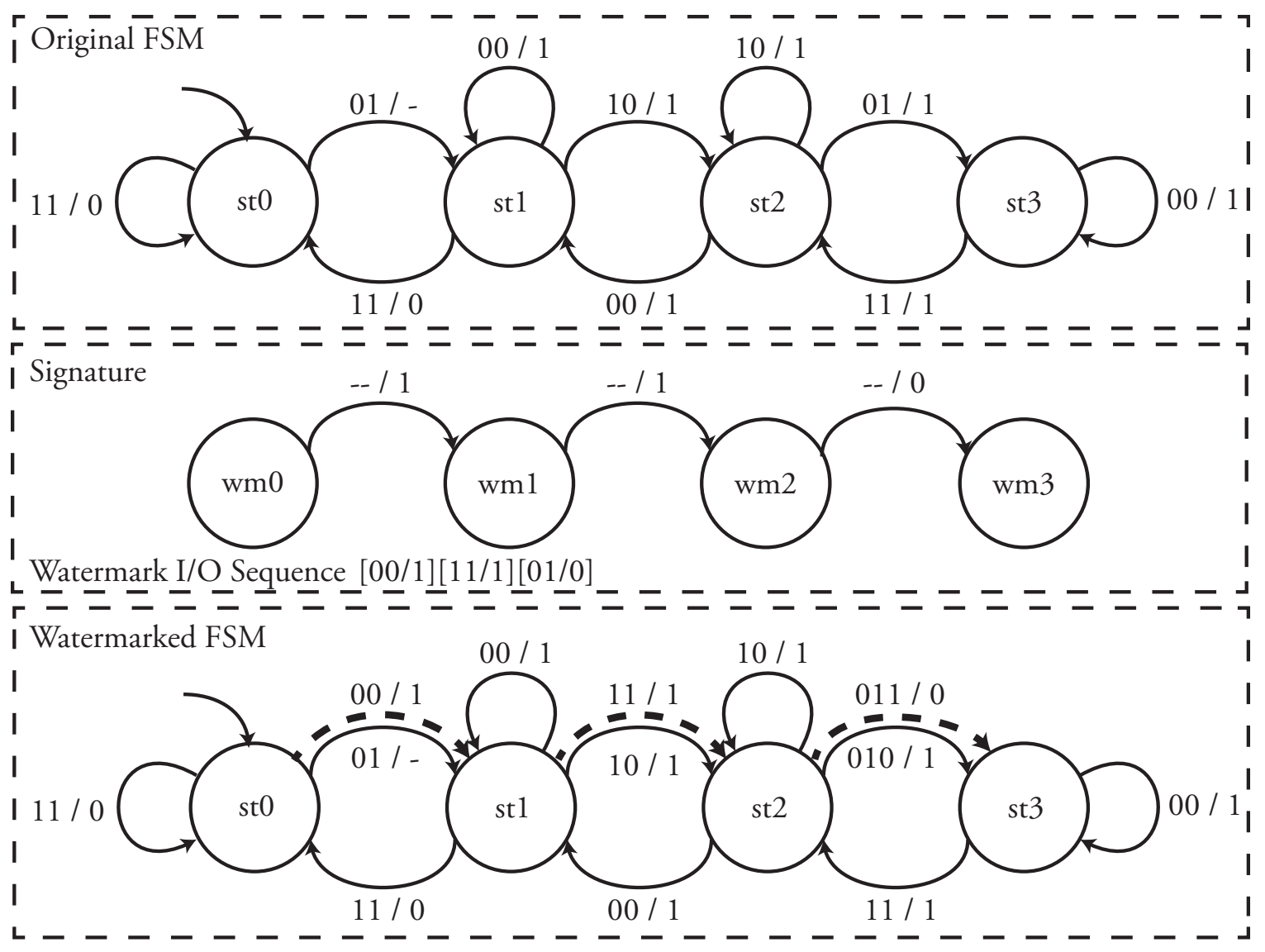

Figure 13: FSM utilizing passive I/O based watermarking

which is known to be Non-deterministic Polynomial Complete (NP-Complete), because the augmenting path utilizes a single state once then the watermark is easily data-mined by finding the set of all edges not contained within the original system. From this, all possible sequences of a given length can be computed starting from any state. This allows desired signatures, different from the original watermark signature, to potentially be constructed. Due to this fact, two separate parties can now claim ownership with no way to distinguish between the "Knight Owner" and the "Knave Owner," or simply no way to distinguish between who is falsely claiming ownership and the real owner.

\subsection{Motivation for This Work}

We begin by recapping the protection methods covered in this chapter and discuss the advantages and disadvantages of each scheme (see Table 3). It can be seen that the majority of the protection based schemes either fall into the two categories, difficult and/or 
costly, and easily compromised and/or removable. This is simply because the more finely tuned the security scheme, the more costly it becomes. Additionally, it can be seen that most of the solutions that have ease of use are also easily compromised.

Table 3: Summary of protection techniques

\begin{tabular}{|c|c|c|c|}
\hline Scheme & IP Type & Advantages & Disadvantages \\
\hline IC Logos & Hard IP & Ease of Use & $\begin{array}{l}\text { Stall Fabrication Runs } \\
\text { Easily Removable } \\
\text { Costly Verification }\end{array}$ \\
\hline Constraint Based & Hard IP & $\begin{array}{c}\text { Ease of Use } \\
\text { Improbable Replication }\end{array}$ & $\begin{array}{c}\text { Many Partial Signatures } \\
\text { Easily Compromised } \\
\text { Difficult to Extract } \\
\text { Costly Verification }\end{array}$ \\
\hline HDL Based & Soft IP & $\begin{array}{l}\text { Obfuscation } \\
\text { Ease of Use }\end{array}$ & $\begin{array}{l}\text { Potentially High Overhead } \\
\text { Easily Compromised } \\
\text { Bitstream Decompilation }\end{array}$ \\
\hline Circuit Based & Soft IP & $\begin{array}{c}\text { Side Channel } \\
\text { Ease of Extraction } \\
\text { Low Overhead }\end{array}$ & Difficult to Design \\
\hline Model Based & Soft IP & $\begin{array}{c}\text { Ease of Use } \\
\text { Ease of Extraction }\end{array}$ & $\begin{array}{c}\text { Easily Removable } \\
\text { Easily Compromised }\end{array}$ \\
\hline
\end{tabular}

Physical protection can be easily removed even when designed for Hard IPs, and owners must resort to expensive methods to verify that the post-fabrication design had not been altered. Similarly, constraint based protection has the advantage of being able to show that replication, by design automation tools, of the watermarked layout is statistically improbable and unique to the owner. Conversely, the simple addition or deletion of an instance will destroy the signature, post-fabrication extraction and verification require costly invasive techniques.

HDL based protection has added benefits such as the ability to obfuscate netlists, making it less intelligible to those licensing the core. However, once compiled into a bitstream format, transforming the Soft IP to a Hard IP, available tools allow for the Hard IPs transformation from bitstream back into a low-level, intelligible, Soft IP format. This allows for HDL designs implementing protection schemes such as Obfuscation and Authentication 
FSMs to become easily compromised and or removed. Additionally, the use of Obfuscation and Authentication FSMs schemes have the potential to generate significant increases in area, based on when the complexity of the protection FSMs is greater when compared to the original system.

Circuit based protection, has extremely desirable benefits, namely, side-channel properties and ease of extraction. Additionally, this type of protection scheme only incurs overhead from the design practices required to implement the glitch logic. In that, altering the design implementation practices to produce otherwise "meta-stable," frequency range based functionality, designs can either increase or decrease the overhead. However, while this type of protection offers many ideal benefits, the main drawback is burdening designers with the daunting task of designing such "meta-stable" circuitry.

Model Based protection, when simply added rather than embedded, is extremely vulnerable to malicious parties during hand-offs in the design process, even in an in-house design setting. This allows for IP cores to become easily compromised by removing the protection schemes added. However, Model Based protection is among the easiest to implement, similar to IC logos, protection schemes can simply be placed before or after the original system had been designed. Alternatively, by embedding, or superimposing, watermarks at the beginning of the design process of models the protection can be increased. Embedding watermarks, helps deter malicious users due to the complexities involved with differentiating between system critical components and the superimposed watermark functionality.

Summarizing the watermarking techniques for sequential systems, we examine the schemes in order as provided in Table 4. Sequential circuit protection schemes can be generalized as one of two methods, the addition of states and the addition of edges and input bits. However, the watermark in all cases is directly output by these schemes. This allows the watermark to be data-mined and easily compromised in a malicious setting. Such that, once an attacker has data-mined additional functionality implemented for the watermark, then the secret sequence required to reproduce the IP owners signature is no longer significant. This is due to the fact that desired signatures can be constructed by utilizing the data-mined information. 
Table 4: Summary of sequential protection techniques

\begin{tabular}{|c|c|c|c|}
\hline Scheme & IP Type & Advantages & Disadvantages \\
\hline State Based & Soft IP & $\begin{array}{c}\text { Ease of Use } \\
\text { Ease of Extraction }\end{array}$ & $\begin{array}{c}\text { Potentially High Overhead } \\
\text { Easily Removable } \\
\text { Easily Compromised }\end{array}$ \\
\hline Edge Based & Soft IP & $\begin{array}{c}\text { Ease of Use } \\
\text { Lower Overhead } \\
\text { Ease of Extraction }\end{array}$ & $\begin{array}{c}\text { Easily Removable } \\
\text { Easily Compromised }\end{array}$ \\
\hline I/O Based & Soft IP & $\begin{array}{c}\text { Ease of Use } \\
\text { Lower Overhead } \\
\text { Ease of Extraction }\end{array}$ & $\begin{array}{c}\text { Easily Removable } \\
\text { Easily Compromised }\end{array}$ \\
\hline
\end{tabular}

State Based watermarking is the simplest technique among the techniques shown in Table 4, and due to the manner in which the technique is accomplished the overhead is likely to significantly increase overall. However, overhead assumptions can be made based on the complexity of the additional FSMs compared to the complexity of the original system. Additionally, data-mining the edges involved in the watermark allow this technique to be compromised. Likewise, because of the addition of separate FSMs, during the design process the watermark can easily be removed.

Edge Based watermarking, like State Based, can easily become compromised through the data-mining of additional system functionality. Similarly, this is due to the fact that the watermark is partially output by the added edges, once a malicious party data-mines the additional edges then ownership potentially becomes indistinguishable. This is because utilizing the outputs from the addition edges desired signatures can be constructed.

Lastly, I/O Based watermarking proves insecure as well. This is from the fact that the watermark is solely based on the creation of additional edges for the desired sequence. Once again, by data-mining the hidden functionality, the watermark can be obtained, compromised, and potentially removed. In this system the watermark can be obtained due to the fact that the watermark signature is based on creating a simple path or cycle and embedding the edges. Thus, the exact watermark signature can be found by simply traversing the set of additional edges and observing the output. 


\subsection{Chapter Summary}

Summarizing this section there are four types of IP protection commonly used: (1) physical protection, (2) HDL protection, (3) circuit protection, and (4) model protection. Physical protection is the use of watermark signatures and/or logos in Hard IP or fabricated ICs. HDL protection is performed at the Soft IP level, through the alteration of hardware netlists for obfuscation, watermarking, or authentication purposes. Circuit level protection is at the gate level, or logic level, of design. It is performed through the use of "Glitch Logic" where the circuity is designed using practices and constraints other than normal to create circuits which will produce a desired glitch output at a given operating frequency. Model level protection is the addition of structures at the highest design level and is illustrated by the techniques presented in this work.

Additionally, there are three types of sequential watermarking techniques commonly used: (1) state based, (2) I/O based, and (3) edge based. State based watermarking is the process of implementing additional states in an FSM which can be used as keys and/or entirely separate functional FSMs. Edge based watermarked is the process of embedding a signature through the use overlapping and created edges which can be used to output part of the signature. I/O based watermarking is the process of embedding a watermark FSM through binding unbound input sequences of an original FSM state to a watermark FSM state. This generates transitions based on unused input sequences to output part of the signature. 


\section{State Encoding Based Watermarking}

In this chapter, we present in detail the proposed watermarking technique that utilizes the FMS's state encoding for the purpose of permanently embedding a digital signature into the FSM. This chapter is organized as follows. In Section 4.2 we present the concept of watermarking via state encoding. In Section 4.4 we present an overview of the proposed watermarking system. Section 4.3 presents experimental costs associated with proposed edge creating techniques. In Section 4.5 we briefly detail the process of the watermark construction phase and present the three proposed methods. In Section 4.5.1 we present in detail the proposed method for generating watermark FSMs from bitmap signatures, Section 4.5.2 presents in detail the proposed method for generating watermark FSMs from file signatures, and in Section 4.5.3 we present in detail the proposed method and custom tool for generating watermark FSMs from hash signatures. In Section 4.6 we discuss the watermark embedding phase of the proposed system and the detailed complexities involved in Section 4.6.1. In Section 4.6.2 we present the proposed brute force solution for watermark embedding and then a greedy heuristic in Section 4.6.3. Section 4.7 details the model generation and verification phase and presents a set of custom tools created in this work. In Section 4.8 we detail the proposed methods for extracting the embedded watermark sequence. Section 4.9 details the security and computational complexities involved for multiple forms of attacks against the proposed method.

\subsection{Note to Reader}

Portions of this chapter have been previously published (Lewandowski et al., 2012)[45] and are utilized with permission of the publisher. 


\subsection{Watermarking via State Encoding}

This method, as previously shown, had not been explored as a watermarking technique for FSMs. From this knowledge we developed a concept that would seamlessly integrate a new level protection into FSMs where the end user would only be impacted by a tolerable cost. By controlling the state encoding values the watermark could be permanently embedded into an FSM and later retrieved when needed. This method currently employs edge creation methods similar to [48] and [50]. New edges created in this method are paired with an unused state input combination, and the output is specified as a don't care condition. This method also utilizes transitions which are known to already provide the desired next state transition, as in [52]. An illustrative example is shown in Fig. 14. We note that in Fig. 14 the original edges are identified by thinner solid lines. Additionally, the watermark edges and overlay edges are identified by thinner and thicker more closely grouped dashed lines, respectively.

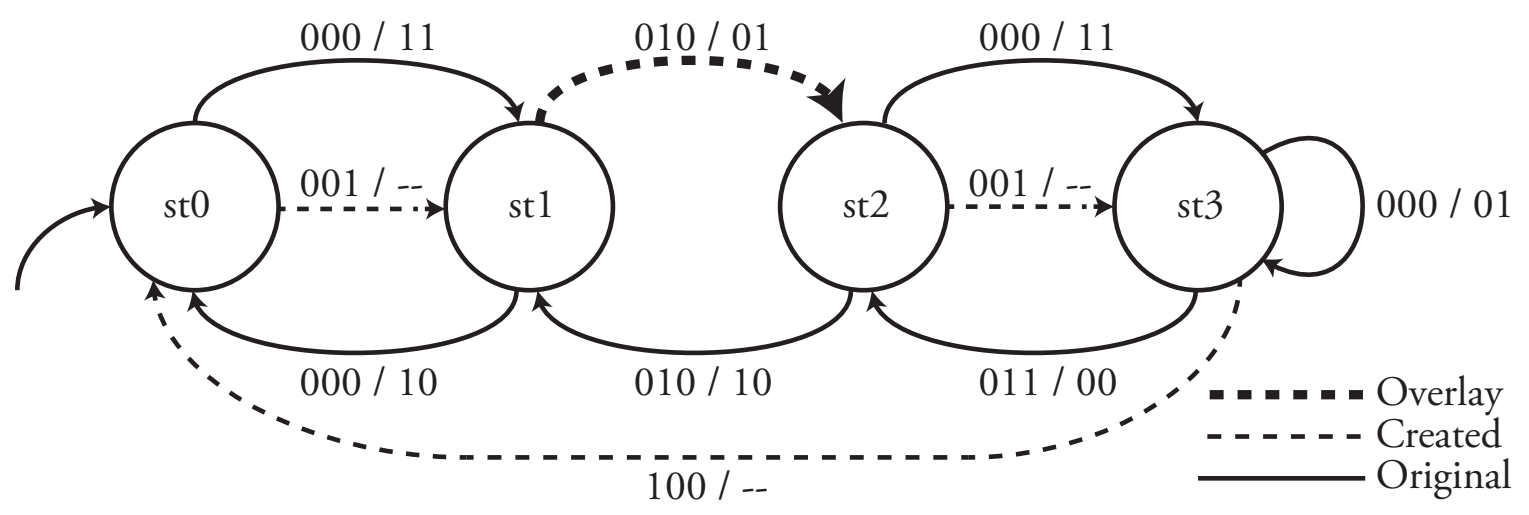

Figure 14: Watermarking edge creation method an illustrative watermarked FSM

\subsection{Edge Creation Cost}

In this section we explore the costs associated with additional edges. We analyze the expected costs of implementing these types of edges by conducting an edge creation related synthesis experiment for the FSM shown in Fig. 14. Using the Xilinx ISE Synthesis tool we synthesized the FSM for a varying number of dummy edges $(0,1,2,3$, and 12) and the results are summarized in Table 5. For more information on full extent of synthesis options utilized see Section 5.2. 
Table 5: Xilinx synthesis results for dummy edges in Fig. 14 FSM

\begin{tabular}{|c|c|c|c|c|c|c|}
\hline Number of Dummy Edges & $\mathbf{( 0 )}$ & $\mathbf{( 1 )}$ & $\mathbf{( 2 )}$ & $\mathbf{( 3 )}$ & $\mathbf{( 1 2})$ \\
\hline States & \multicolumn{5}{|c|}{4} \\
\hline Transitions & 11 & 12 & 13 & 14 & 23 \\
\hline Input Bits & 3 & 4 & 5 & 8 \\
\hline Output Bits & \multicolumn{5}{|c|}{4} \\
\hline Encoding & \multicolumn{5}{|c|}{ LUT } \\
\hline Implementation & \multicolumn{5}{|c|}{2} \\
\hline Registers Used & \multicolumn{5}{|c|}{1075.963} \\
\hline Look-Up Tables (LUTs) Used & \multicolumn{5}{|c|}{} \\
\hline Max. Frequency (MHz) & \multicolumn{5}{|c|}{} \\
\hline
\end{tabular}

For this experiment, we initially synthesized the original FSM in Fig. 14, i.e., without any additional dummy edges. These results are reported in column (0) in Table 5. We then began adding the non-overlay edges from Fig. 14 one-by-one performing synthesis after each addition. These results are reported in columns (1), (2), and (3) in Table 5. Once we had synthesized the original FSM, the example watermarked FSM, we then examined the synthesis results for the scenario where the number of edges added doubled the number of edges in the original FSM. This synthesis results for this scenario are reported in Table 5 as column (12). From the data presented in Table 5 it can be seen that the Xilinx Synthesis results, for this example, returned potentially promising implementation cost values.

\subsection{Watermarking System: Overview}

The watermarking system that was created utilizes a variety of tools for accomplishing the task at hand. At the highest level, the system has three major phases, see Fig. 15, (a) Watermark Construction Phase, (b) Watermark Embedding Phase, and (c) Model Generation \& Verification Phase. The Watermark Construction Phase transforms the desired signature into a graph. In the Watermark Embedding Phase the signature graph is embedded into the FSM by overlaying it into nodes and edges of the STG representation of the FSM. If necessary new edges are created. In the Model Generation and Verification 
Phase, the modified FSM is converted into a testable HDL model and verified. Below we provide more details of each phase with illustrative examples.

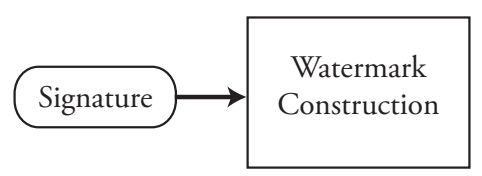

(a) Construction phase

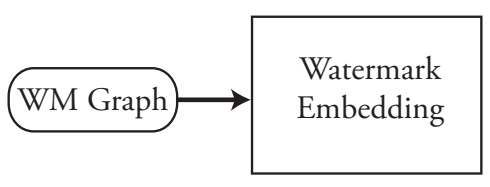

(b) Embedding phase

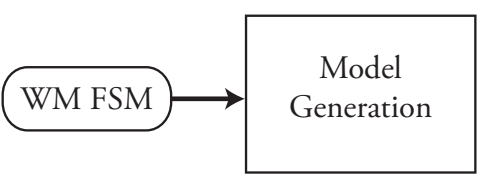

(c) Modeling phase

Figure 15: High level overview of watermarking system flow

\subsection{Watermark Construction Phase}

The method for constructing the watermark has continuously evolved throughout the life of this work. We propose three techniques ranging from the utilization of bitmaps to signature hashing. The proposed watermark construction methods are shown in Figs. 16 (a), (b), and (c). Below we propose each watermark construction method by providing an algorithm for the technique and possible examples of signatures, decomposition, and sequences. We also discuss advantages and disadvantages of each method.

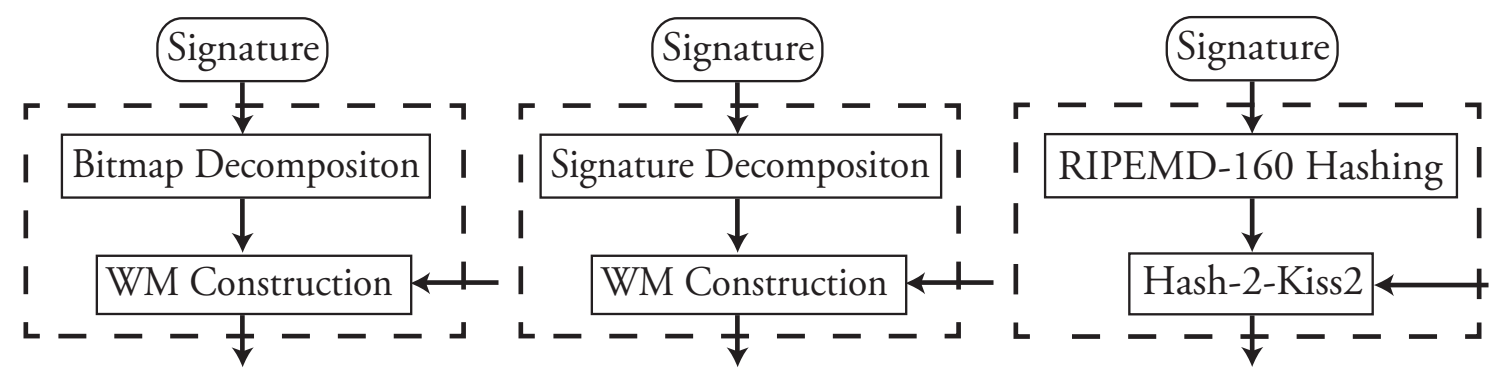
(a) BSD method
(b) FSD method
(c) HSD method

Figure 16: Methods of watermark construction

\subsubsection{Bitmap Signature Decomposition}

BSD is the first form of watermark construction implemented in this work. BSD was employed for the ease in constructing and verifying a proof of concept model. To illustrate this, Fig. 17 provides a sample bitmap signature that was used in the BSD method. From this, using the BSD method, the bitmap signature would be decomposed into a raw binary 
format. We note that black squares in the bitmap are represented by logical ones, while white squares are represented by logical zeros. From this simplistic binary encoding scheme there exist a plethora of viable methods for the bitmap decomposition. Arbitrarily, we chose the simplest method for decomposing the signature, which was a row-concatenate based implementation. This algorithm operates by traversing the bitmap in a raster scan fashion to yield a single binary sequence. The concatenated string is split into chunks equal to the length of the state encoding of the FSM. If the last chunk is not appropriately sized the remaining bits are LSB sign extended with logical zeroes until the appropriate chunk size has been met. LSB sign extension is used as to not compromise the signature, i.e., while " $10 "$ and " $100 "$ are not binary equivalent, when the signature is constructed it will simply have an extra "0" at the end of the reconstructed signature. For example, if the signature sequence is "11110," with a block size of three, inserting a leading zero would now compromise the signature and generate " $111|0| 10$ " instead of the original desired signature "11110|0|." The worst case run-time for composing the single concatenated signature string is $\mathcal{O}(n \times m)$, where $n$ is the standardized length of each row, and $m$ is the number of rows used total. However, because the sequence is concatenated into a single string, the worst case run-time of chunk creation is $\mathcal{O}(x)$, where $x$ is the length of the concatenated string. This is because all of the operations for constructing the blocks are based off of constant, or other linear time growth, operations in the code. Which overall, causes this method to entail a worst case run-time of $\mathcal{O}(n \times m)$, which is the time for computing single concatenated bit string. From this, the method would prove to be rather simplistic and ideal complexity and run-time for decomposing signatures. Unfortunately, the main drawback of this method is the lack of signature flexibility. In addition, this method would only work under the condition that bitmap image signatures were not artistically intricate in their design.

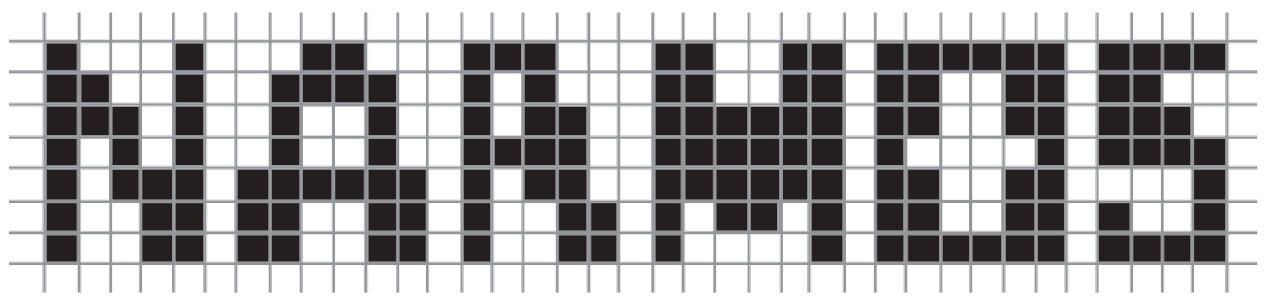

Figure 17: Sample bitmap signature 


\subsubsection{File Signature Decomposition}

FSD operates in the same manner as BSD and the only difference is that this concept has been extended to digital files. By transforming a file into a machine level representation we are able to construct the raw binary representation of the signature. This enables us to utilize any format, ranging from text files to audio clips, as a watermark signature. However this can become a very lengthy and time consuming process due to the massive amount of raw data. We illustrate this by presenting a small fraction of the amount of data in a sample file in Table 6 .

Table 6: Sample file under FSD

\begin{tabular}{|c|c|c|c|c|c|}
\hline \multicolumn{7}{|c|}{ Simple VHDL File } \\
\hline File Size: 9.98 KB (10,222 bytes) \\
\hline Row Address & & 1 & $\cdots$ & $E$ & $F$ \\
\hline & 0 & 00101101 & $\cdots$ & 00101101 & 00101101 \\
\hline 00000000 & 00101101 & $0010 \mathrm{mn}$ Address \\
\hline 00000020 & 00101101 & 00101101 & $\cdots$ & 00101101 & 00101101 \\
\hline 00000030 & 00101101 & 00101101 & $\cdots$ & 00101101 & 00101101 \\
\hline 00000040 & 00101101 & 00101101 & $\cdots$ & 00101101 & 00101101 \\
\hline
\end{tabular}

For the sake of clarity, we note that we did not include the entirety of the files contents, and that all of the presented row \& column binary bit sets only represent about half of a single line of text in the original file, it is also noted that for brevity we did not include the entire column range. However, this file contains roughly 640 more row addresses similar to those shown in Table 6 . From this, it can be seen that the amount of data to be processed, bit by bit, grows rather rapidly for what modern computer users would see as an insignificantly sized file. Nonetheless FSD, similarly to its BSD predecessor, also needed to employ the use of sign extension when the data at a given row address ended before column $F$, or when the block size specified would create inappropriately sized chunks. The solution for this sign extension process was performed in the same manner of BSD, with the exception that, columns were filled with "00000000" until the appropriate size was reached. This appropriate size for sign extension was determined simply by the number of 
bits required to size the last chunk in the system based on the provided block size. This process of extension, and its resulting behavior, is acceptable due to the fact that writing zeroes to the end of the file will not actually alter or compromise a signature in most cases, but instead, it will only slightly increase the size of the signature, further making. Whether or not the extension of zeroes visually alters a file, in the typographical sense, depends on whether or not a utilized text editor will show characters representing this extension. For example, a popular open source tool Notepad++ [70] will display "NUL" characters which represents this extension of zeroes. While the standard Windows Notepad will keep this as a seemingly invisible alteration. From this the chunks would be computed as they were in BSD by using the predetermined block size. Similarly the worst case run-time calculations are also the same. However, due to file size complexities, constructed watermarks were almost always large completely specified state machines. This left little flexibility in embedding and resulted in high overhead. This is further illustrated by an example of FSD in Fig. 18. It can be seen that a relatively small file, Fig. 18(a), under FSD can generate a completely connected signature, Fig. 18(b), which can become extremely costly to implement as a signature.

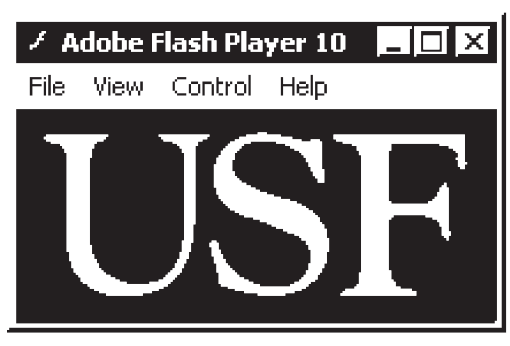

(a) Signature used in FSD

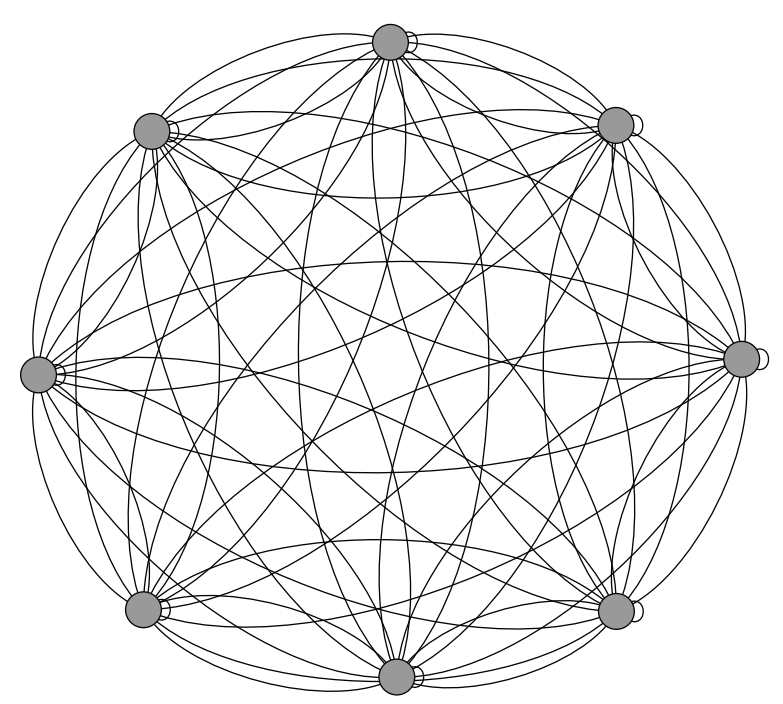

(b) Corresponding STG

Figure 18: Example of FSD 


\subsubsection{Hashing Signature Decomposition}

From the ideal conditions of the BSD method, and rather poor outcomes illustrated by FSD, alternative solutions for signature decomposition were needed. Through this search gave rise to the current decomposition method, which is known as HSD. This method employs the external use of a secure hashing function to uniquely hash the signature intended to be used in watermarking. We chose to utilize the secure hashing function RACE Integrity Primitives Evaluation Message Digest 160-bit (RIPEMD-160) [71] because it is a hashing algorithm that returns a shorter 40 character hash sequence and has been shown to be collision free [72]. The signature chosen for illustration of this method was the Portable Network Graphic (PNG) file shown in Fig. 19. Using the OpenSSL tool [73] and RIPEMD160 hash to digest this signature, the corresponding hash value was returned:

\section{$" R I P E M D 160(C O E-h$-black.png $)=91$ efebee4bd48a62f338f244560b668771fa338e"}

This hash, in bold, would then be transformed from its present hexadecimal format into a decimal representation that could be further utilized in an STG format. To perform this process we created the Hash-2-K2 program, which will automatically manipulate the hash sequence and generate a resulting watermark STG in Kiss2 format. The benefits of this method are that any hashing function can be used in the HSD method, while OpenSSL offers a variety of hashing solutions, one can still obtain even greater flexibility by utilizing a hash of choice. However, the run-time of the HSD method is solely dependent of the hashing algorithm chosen for use, while the transformation of the hash and watermark construction run-times are covered in Section 4.5.4, as these manipulations are performed by the Hash-2-K2 tool.
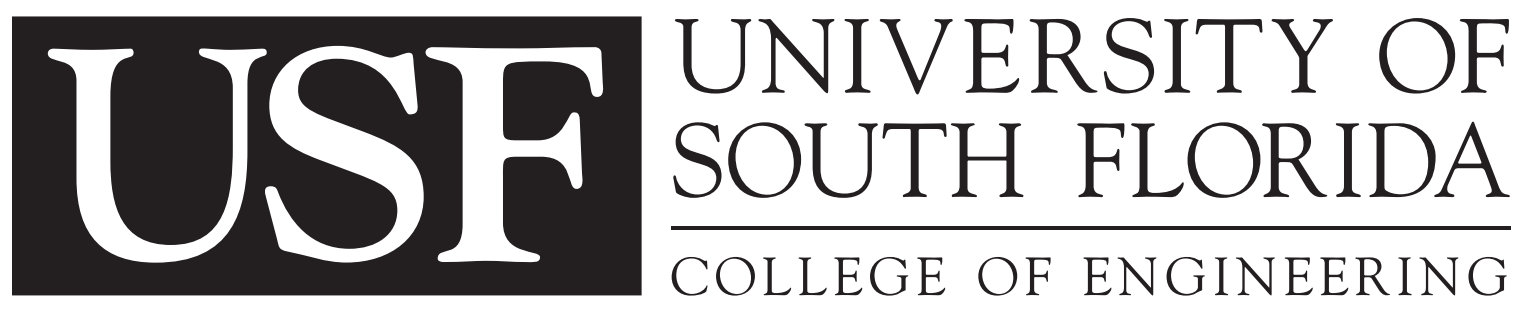

Figure 19: Sample HSD signature prior to hashing 


\subsubsection{HSD Watermark Construction: Hash-2-K2}

Once the signature has been decomposed, it is ready to be further manipulated into a format that could be used for watermarking. In this section we will cover the current implementation of watermark construction that is achieved by the Hash-2-K2 program which we have developed as part of this thesis work. The standard input to the program is the previously illustrated hash output format from OpenSSL in Section 4.5.3. First, each hexadecimal hash value is converted into the corresponding decimal representation. This decimal representation is used as denoting a node in the watermark graph. From this new representation an adjacency matrix is constructed for the hash, we define a hash adjacency to be the left and right neighbors of a single character, i.e., in the sequence "91e," "1" is adjacent to both "9" and "e." Once the adjacency matrix is created, we generate the corresponding Kiss2 format file for the hash. This enabled us to generate sparser graphs than those from the FSD and BSD method while increasing the signature complexity. Using a tool called "k2net," which utilizes the Pajek netlist format [74] and Gephi [75], an open source graph visualization and manipulation software, we verified the correctness of the generated graph. The RIPEMD-160 Hash graph is shown in Fig. 20.

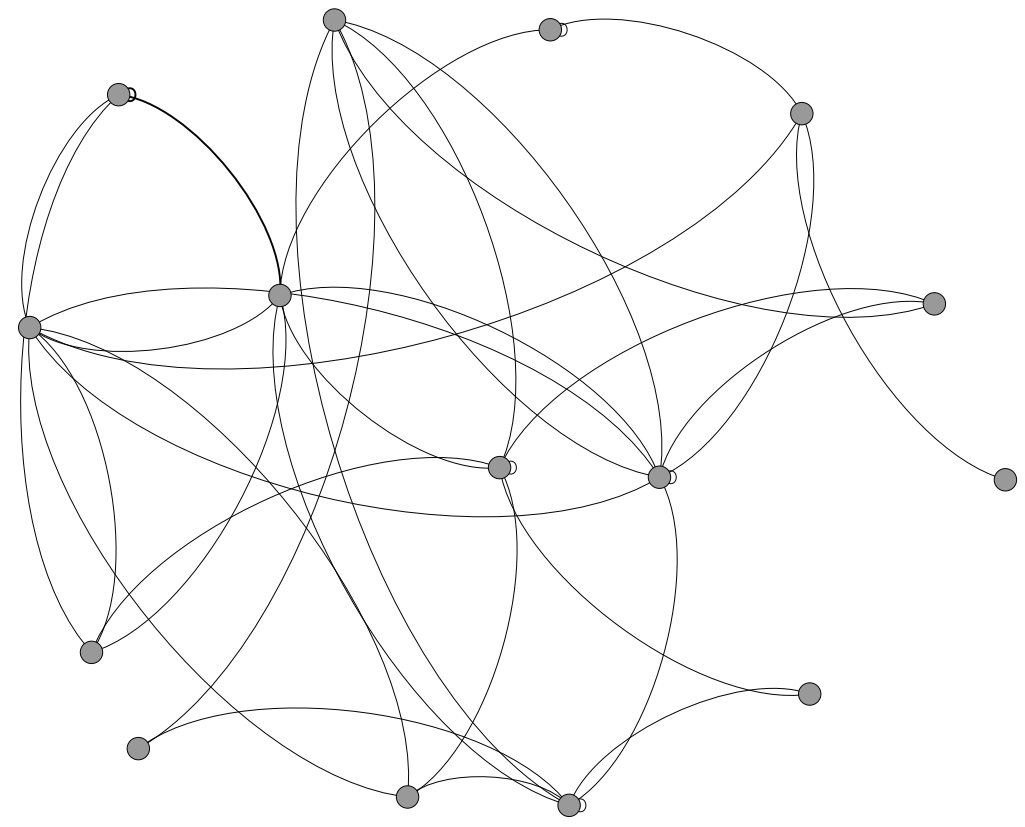

Figure 20: RIPEMD-160 Pajek netlist / STG using Gephi 
We note that for the sake of clarity, edges and vertices are not labeled in Fig. 20, rather we illustrate the concept of turning a hash into an undirected graph for the purpose of embedding. Now that the hash has been converted into a format that can be processed by the watermarking tool we can proceed forward. The worst case run-time of this format conversion algorithm is dictated by the construction and processing of the adjacency matrix. Thus the worst case run-time of the Hash-2-k2 tool will be $\mathcal{O}\left(n^{2}\right)$, where $n$ is the number of unique values that represent states in the hash. We also note that the worst case run-time of the k2net tool, used for generating visualizations of $\mathrm{k} 2$ files, is $\mathcal{O}(x)$, where $x$ represents the number of edges in the STT format. This is due to the fact that the STT format can be traversed in an row based manner for writing the corresponding Pajek netlist information to the file.

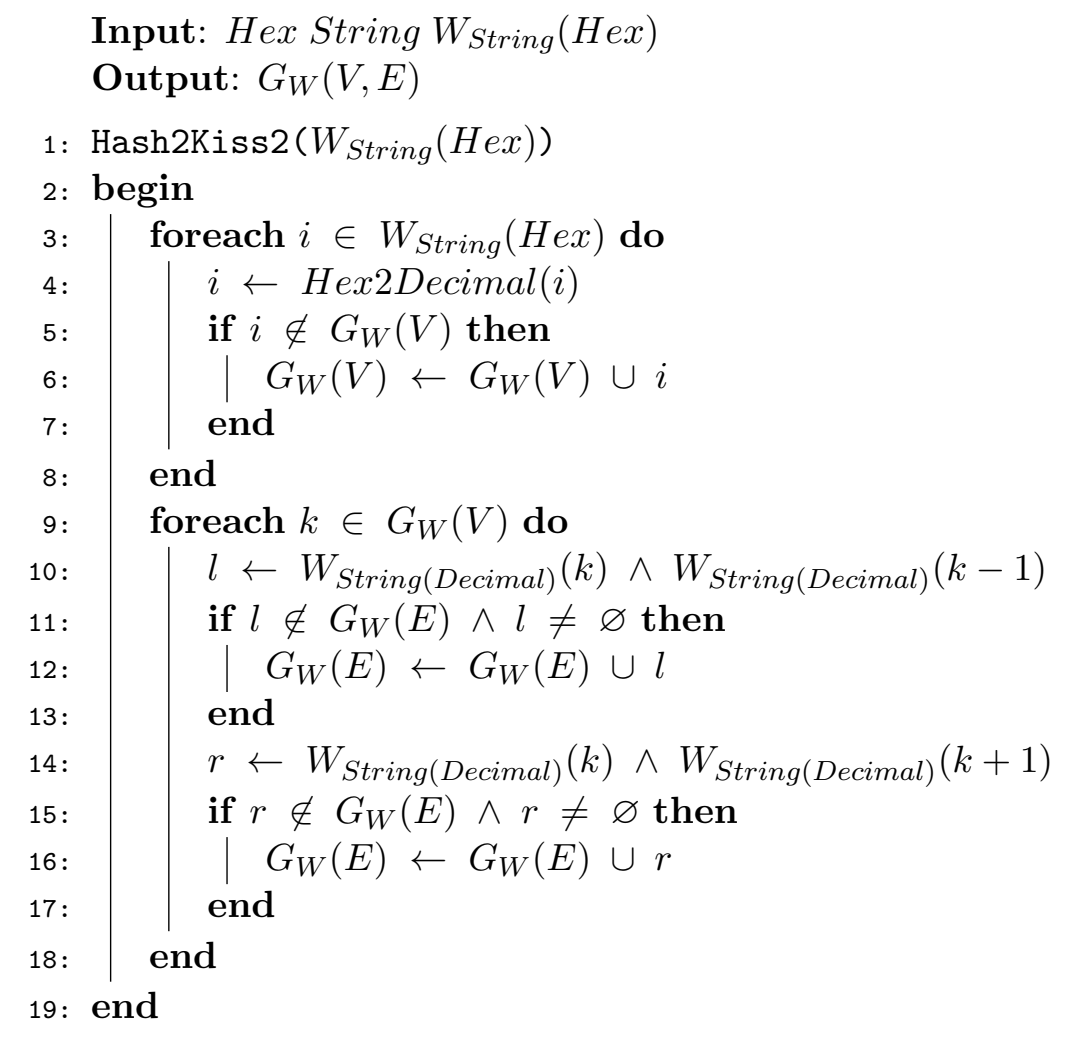

Figure 21: Hash-2-Kiss2 watermark construction algorithm

The psuedo-code of the Hash-2-Kiss2 watermark construction algorithm is shown in Fig. 21. The process accepts a string in hexadecimal format (line 1), and while the string is not empty the process converts each of the hexadecimal values to their respective decimal 
value, i.e., "A" maps to "st10" (line 4). Additionally, the algorithm will only add unique states to the graph, such that, if the hexadecimal character "A" happens to appear more than once, then it is only added to $G_{W}(V)$ once (line $5 \& 6$ ). Similarly, if there exists an edge which connects two known states in the string and is not already an element in $G_{W}(E)$ this edge is added (line $8 \& 9$ ). This prevents redundant edges from being added to the watermark FSM and creating addition overhead in the embedding process, for example if the sequence is "9AABC10AA," the sequence "AA" appears twice but is only added the first time it is found in the sequence.

\subsection{Watermark Embedding Phase}

The Watermark Embedding Phase is the second phase where in the original STG is watermarked with the undirected graph of the hash signature. In this section, we cover the complexity of the problem and proposed algorithms.

\subsubsection{Embedding: Complexity}

The task of embedding one graph into another is directly related to the sub-graph isomorphism/matching problem. This sub-graph matching problem is to determine if there exists a possible configuration of a graph, $\operatorname{Graph}_{A}$, which is contained, or can be mapped, as a subset in another graph, $\operatorname{Graph}_{B}$. In relating this to our problem, we are seeking a low cost configuration represented by $G_{W}(V, E) \subset G_{F S M}(V, E)$, where $G_{W}(V, E)$ and $G_{F S M}(V, E)$ are, respectively, the watermark and original FSM graphs. However, if an inherent sub-graph has been found in an unaltered FSM then the watermark is lost to the normal functionality of the machine, and an alternative signature must be sought for use. Alternatively, if $G_{W}(V, E) \not \subset G_{F S M}(V, E)$, then an inherent isomorphic sub-graph has not been found, and in our case the appropriate actions must be taken. However, from this generalized overview the complexity can be derived through its matching relation to the clique problem, where the goal is to find complete sets in which each element is connected [76]. This is ultimately done by finding a clique of nodes in $G_{F S M}(V, E)$ that can be identically, or by low cost means in our case, mapped to $G_{W}(V, E)$. The clique problem 
known to be NP-Complete [77]. Thus, there is potentially no efficient solution for tackling this problem in a reasonable amount of time.

\subsubsection{Brute Force Embedding Algorithm}

Determining the number of mapping combinations is specifically dependent on both the watermark and original FSM graphs. However such a method for generating all mapping combinations is considered to be an r-Permutation, and can be computed from equation 3 , and is specifically for situations where $n$ choices can be mapped to $r$ positions [78]. In our case, we consider the number of states in the original FSM to be $n$ choices which can be mapped to one of $r$ positions in the watermark, where $r$ is the number of states in the watermark graph.

$$
P(n, r)=\frac{n !}{(n-r) !}
$$

Thus, the first, proof of concept, algorithm exhaustively checks r-Permutations by Brute Force, and is suitable for small state machines. The algorithm employs a recursive paradigm to generate all possible state matching combinations. Through the use of a cost calculation function the algorithm selects the lowest cost mapping for the watermark graph to the original FSM.

This brute force algorithm, shown by the pseudo-code in Fig. 22, accepts both the original $\mathrm{FSM}, G_{F S M}(V, E)$, and watermark graph, $G_{W}(V, E)$, additionally, the algorithm also accepts an initial match set. The match set contains a unique permutation of mappings, or ordered pairs, from states in the original FSM and corresponding watermark FSM states, or $G_{W}(V) \rightarrow G_{F S M}\left(V^{\prime}\right) \subset G_{F S M}(V)$, and is initially empty until the algorithm first inserts a mapping (line 8). Initially, the best cost is set to the size of the edge set, or number of edges, in the watermark graph (line 3). For each state in the original FSM (line 4) and each state of the watermark FSM (line 5), the algorithm will add a mapping of an original FSM state to a watermark FSM state (line 6). Once adding this mapping, the algorithm then checks whether or not the removal of the previously mapped watermark FSM state will cause the set of all watermark FSM states to become empty (line 7). If this set is not empty then the algorithm will recursively call itself providing 


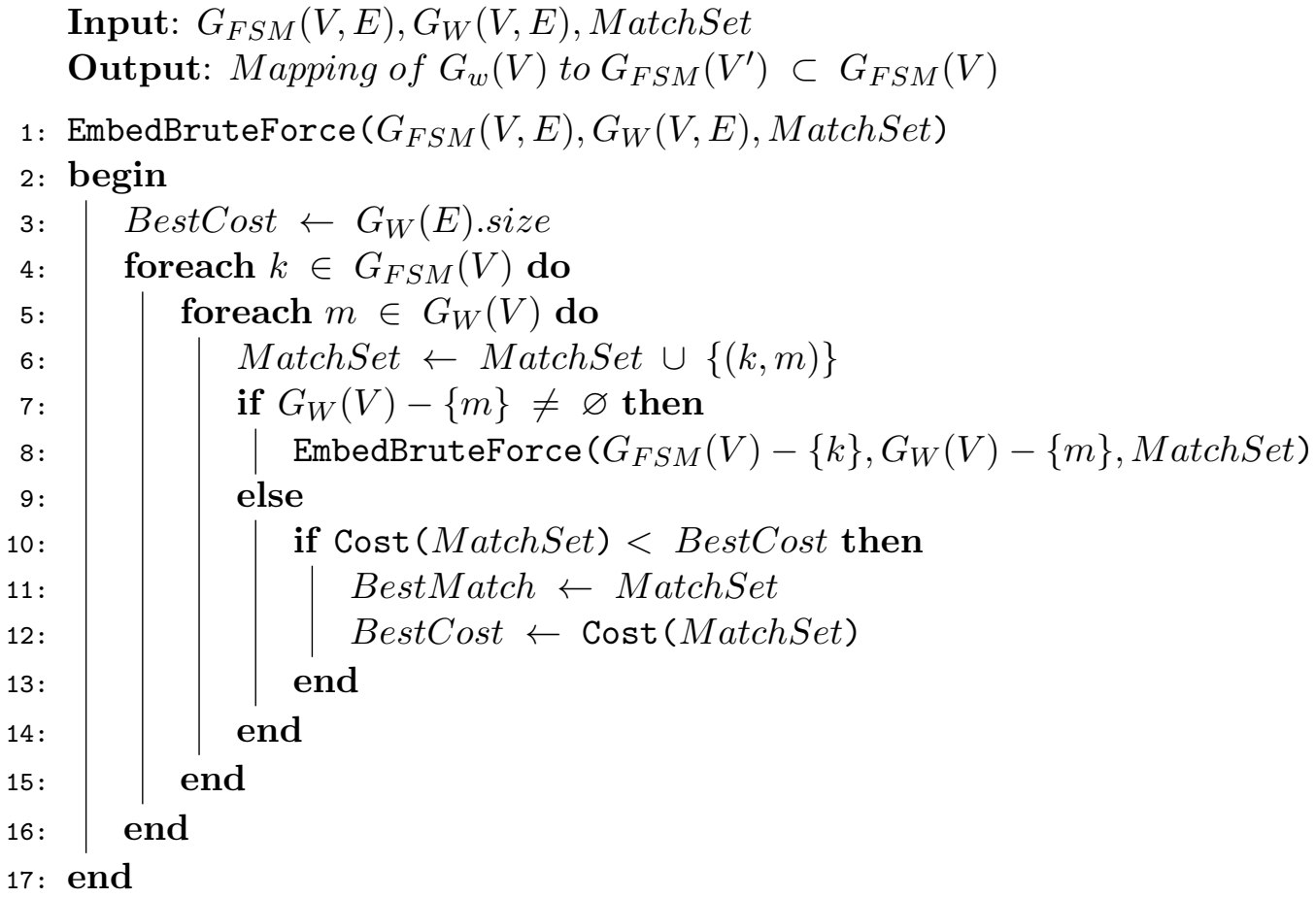

Figure 22: Proposed brute force watermark embedding algorithm

the current set of matches with the removal of the current original FSM state, $k$, and the current watermark FSM state, $m$, (line 8). However, if this removal causes the set to become empty, such that all watermark FSM states have been mapped, then the algorithm will calculate the cost of the current match comparing it to the cost of the known best cost (line 10). Figure 23 shows the pseudo-code for calculating the cost of the mapping (line 9) in the brute force algorithm, Fig. 22. We note that $M_{(k, m)}$ is the relative difference between the set of edges for the mapped graph nodes $G_{F S M}(k)$ and $G_{W}(m)$, formally, $M_{(k, m)}=\left\{x \in U \mid x \notin G_{F S M}(k) \wedge x \in G_{W}(m)\right\}$, where $U$ is the set of all edges [78]. If the returned calculated cost, of the current match set, is lower than the currently known best cost, the best match and best cost are updated.

To further illustrate the brute force embedding algorithm, we offer an example of the algorithm embedding the watermark FSM, shown in Fig. 24(b), into the original FSM shown in Fig. 24(a). For the sake of clarity we do not label transitions, additionally, alphabetic state encoding values and numerical state encoding values are respectively assigned to the original and watermark FSM. The set of mapping combinations, or simply all permutations 
Input: MatchSet

Output: Costof MatchSetMapping

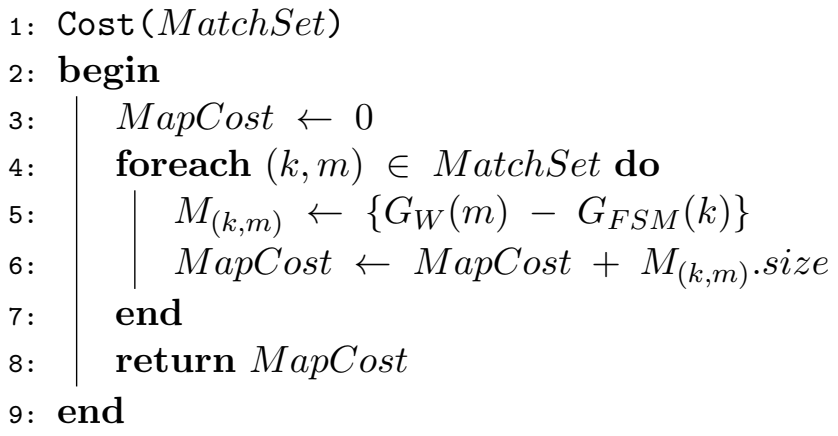

Figure 23: Brute force cost calculation algorithm

of the match set values, generated by the brute force algorithm is provided by Table 7 . By equation 3 , for this example, the expected number of mapping combinations is 24 , which is additionally verified by Table 7 .

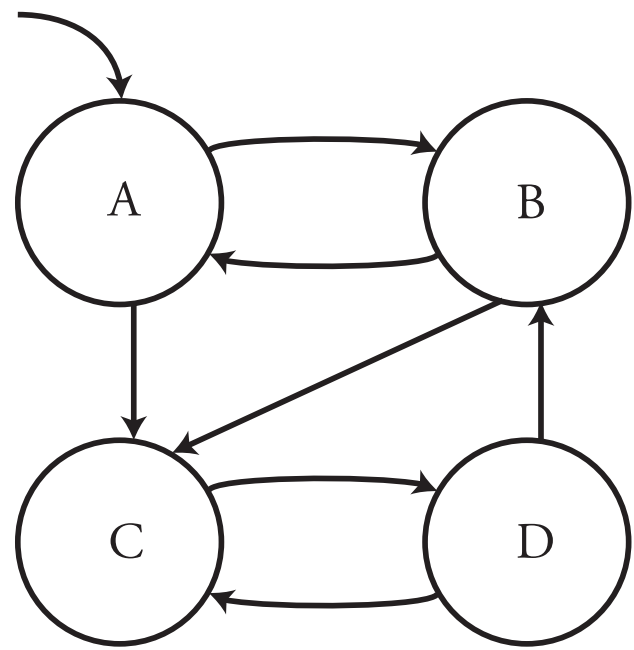

(a) Original FSM

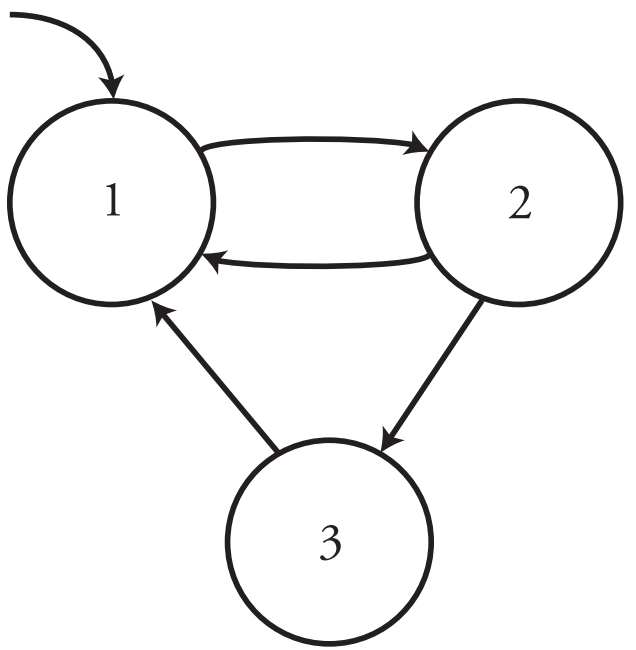

(b) Watermark FSM

Figure 24: Original and watermark FSMs for brute force embedding example

Each of the match sets, shown in Table 7, has its associated mapping cost calculated and compared against the known best. These corresponding mapping costs, calculated by the algorithm in Fig. 23, will be compared in order throughout the algorithm. Shown in Table 8 are the map costs and actions taken by the algorithm for the corresponding range sets in Table 7. 
Table 7: Brute force mapping combinations

\begin{tabular}{|c|c|c|c|c|}
\hline \multirow{4}{*}{$G_{F S M}(A, B, C, D) \rightarrow G_{W}(1,2,3)$} & \multicolumn{4}{|c|}{ Combinatorial Ranges } \\
\cline { 2 - 5 } & {$[1-6]$} & {$[$ 7-12] } & {$[13-18]$} & {$[19-24]$} \\
\hline & $(\mathrm{A}, \mathrm{B}, \mathrm{C})$ & $(\mathrm{B}, \mathrm{A}, \mathrm{C})$ & $(\mathrm{C}, \mathrm{A}, \mathrm{B})$ & $(\mathrm{D}, \mathrm{A}, \mathrm{B})$ \\
\cline { 2 - 5 } & $(\mathrm{A}, \mathrm{B}, \mathrm{D})$ & $(\mathrm{B}, \mathrm{A}, \mathrm{D})$ & $(\mathrm{C}, \mathrm{A}, \mathrm{D})$ & $(\mathrm{D}, \mathrm{A}, \mathrm{C})$ \\
\cline { 2 - 5 } & $(\mathrm{A}, \mathrm{C}, \mathrm{B})$ & $(\mathrm{B}, \mathrm{C}, \mathrm{A})$ & $(\mathrm{C}, \mathrm{B}, \mathrm{A})$ & $(\mathrm{D}, \mathrm{B}, \mathrm{A})$ \\
\cline { 2 - 6 } & $(\mathrm{A}, \mathrm{C}, \mathrm{D})$ & $(\mathrm{B}, \mathrm{C}, \mathrm{D})$ & $(\mathrm{C}, \mathrm{B}, \mathrm{D})$ & $(\mathrm{D}, \mathrm{B}, \mathrm{C})$ \\
\cline { 2 - 5 } & $(\mathrm{A}, \mathrm{D}, \mathrm{B})$ & $(\mathrm{B}, \mathrm{D}, \mathrm{A})$ & $(\mathrm{C}, \mathrm{D}, \mathrm{A})$ & $(\mathrm{D}, \mathrm{C}, \mathrm{A})$ \\
\hline & $(\mathrm{A}, \mathrm{D}, \mathrm{C})$ & $(\mathrm{B}, \mathrm{D}, \mathrm{C})$ & $(\mathrm{C}, \mathrm{D}, \mathrm{B})$ & $(\mathrm{D}, \mathrm{C}, \mathrm{B})$ \\
\hline
\end{tabular}

Table 8: Brute force cost mapping combinations

\begin{tabular}{|c|c|c||c|c||c|c||c|c|}
\hline & \multicolumn{7}{|c|}{ Combinatorial Ranges } \\
\hline Cost[0] = 4 & {$[1-6]$} & Action & {$[$ 7-12] } & Action & {$[13-18]$} & Action & {$[19-24]$} & Action \\
\hline \hline \multirow{3}{*}{ Cost } & 1 & Update & 1 & N/A & 1 & N/A & 3 & N/A \\
\cline { 2 - 9 } & 2 & N/A & 1 & N/A & 2 & N/A & 2 & N/A \\
\cline { 2 - 9 } & 2 & N/A & 2 & N/A & 1 & N/A & 2 & N/A \\
\cline { 2 - 9 } & 3 & N/A & 1 & N/A & 2 & N/A & 1 & N/A \\
\cline { 2 - 9 } & 3 & N/A & 2 & N/A & 1 & N/A & 2 & N/A \\
\cline { 2 - 8 } & 3 & N/A & 2 & N/A & 0 & Update & 2 & N/A \\
\hline
\end{tabular}

Upon completion of the algorithm the map set with the lowest cost, in this example $(\mathrm{C}, \mathrm{D}, \mathrm{B})$ with a cost of 0 , is returned. From this match set, returned by the brute force embedding algorithm, the resulting watermarked FSM is then generated. Additionally, the resulting watermarked FSM for this example, generated from the original and watermark FSMs, Fig. 25(a) and Fig. 25(b), is shown by Fig. 25(c).

Run-time analysis of this algorithm shows that $k$ ! iterations will recursively call the function EmbedBruteForce() a total of $m$ ! times during which the algorithm will construct all combinations of possible match sets. Due to this recursive nature of the algorithm worst case run-time complexity can be observed as $\mathcal{O}\left({ }^{n} P_{m}\right)$, where $k$ and $m$ are the number of original states and watermarking states respectively. Alternatively, this worst case run-time is also given by equation 3 .

The drawbacks of the brute force algorithm are: (1) excessive run-time, and (2) lack of scalability. Based on worst case run-time calculations it is apparent that this system 


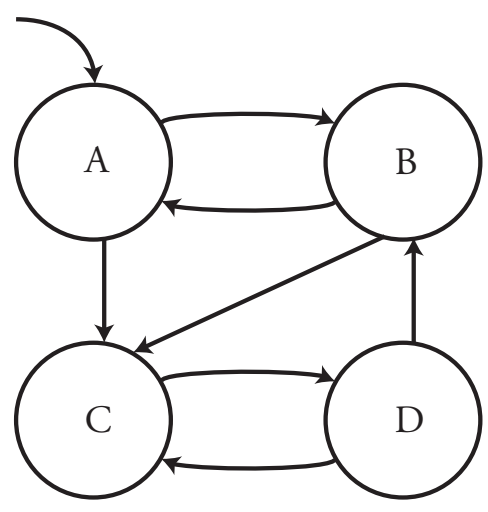

(a) Original FSM

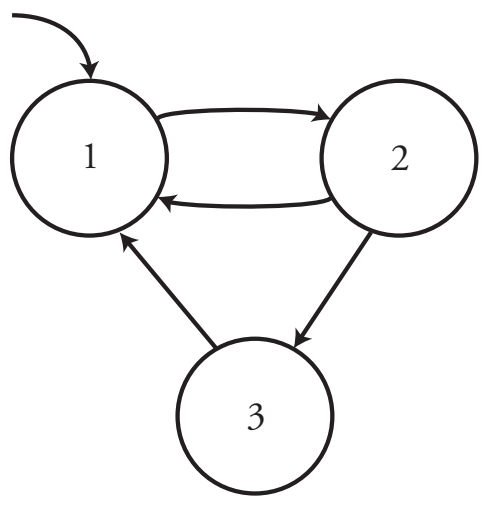

(b) Watermark FSM

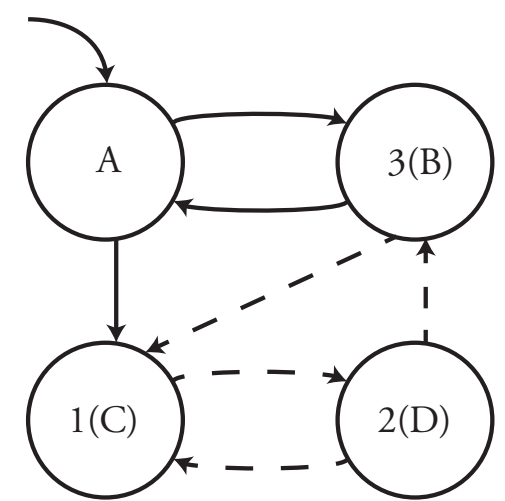

(c) Watermarked FSM

Figure 25: Original, watermark, and watermarked FSMs for brute force embedding

will lack desired scalability due to the complexity of generating all possible permutations. Typically brute force solutions aren't sought out for this reason. From this, the need arose for a more efficient algorithm that could be easily scaled for FSMs ranging in size, with significantly lower run-time, and didn't require the search space to be exhaustively explored.

\subsubsection{Greedy Embedding Algorithm}

While it is generally known that greedy algorithms (with few exceptions, such as Hu's scheduling algorithm [79]), are unlikely to produce globally optimal solutions. The proposed algorithm utilizes a node based complexity cost calculation for both watermarked and un-watermarked states, which is used in the process of determining potential state matches. The equations used for calculating the associated cost calculation for a single state in either of the two machines and the cost associated with mapping a watermark state to an original FSM state are given by equations 5 and 4 respectively. Alternatively, we note that equation 4 is simply the degree of a node. Using these equations the algorithm first calculates the cost of each node in the FSM and in the watermark.

$$
\begin{gathered}
\text { Node Cost }=\left(G_{F S M}, V_{\chi}\right)=\left(V_{\chi}\right)_{\text {in }}+\left(V_{\chi}\right)_{\text {out }} \\
\text { Map Cost }=\text { Node } \operatorname{Cost}\left(G_{F S M}\left(V_{\chi}\right)\right)-\operatorname{Node} \operatorname{Cost}\left(G_{W}\left(V_{\chi^{\prime}}\right)\right)
\end{gathered}
$$


Through the use of this newly calculated cost information the algorithm selects and maps the lowest cost implementation between the current watermark state and best match FSM state. It repeats this process for each unmatched watermark node by traversing the edges connected to the initial match while proceeding to search for the next lowest mapping. The pseudo-code for this algorithm is shown in Fig. 26.

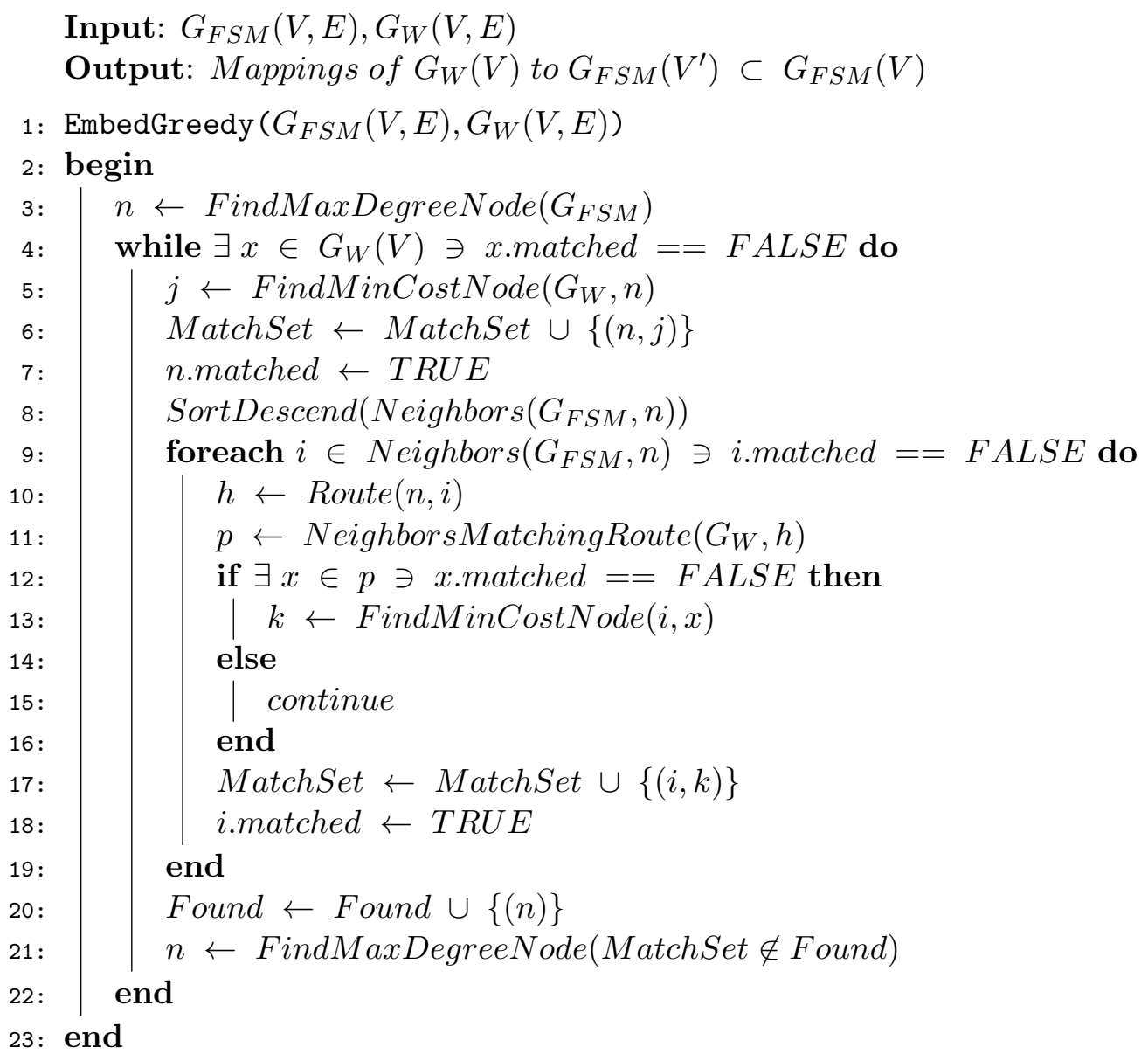

Figure 26: Greedy heuristic

The greedy algorithm initially accepts the original and watermark FSMs (line 1). The algorithm begins by finding the maximum degree node in the original FSM (line 2), by calling the FindMaxDegreeNode() function that is shown in Fig. 27. The greedy algorithm is then run until there exists a node in the watermark FSM such that it hasn't been matched (line 4). Beginning the loop the algorithm finds the minimum cost node, in the watermarking FSM, that can be paired with the recently found highest cost node (line 5) by calling the function FindMinCostNode(). This function is shown by Fig. 28. 
The match set is then updated (line 6) with the previously returned minimum cost node from FindMinCostNode() and then (line 7) $n$ is marked as being matched. Following, the unmatched nodes are sorted in descending order first by calling the Neighbors() function and passing the returned list to the SortDescend() function (line 8). These two functions are shown in Fig. 29 and Fig. 30. The algorithm then checks each of the neighbors in descending order that are still unmatched (line 9). Following this, the direction of the edge between the unmatched neighbor and the current node determines if it is an incoming or outgoing edge (line 10). From this direction the algorithm finds additional neighbors matching this edge route (line 11). If there is a neighbor which matches the edge route and is still unmatched then algorithm calls the FindMinCostNode() function and maps it to a node on the connected path (lines $12 \& 13$ ). If there are currently no unmatched nodes then the algorithm continues (line 15). Following this, the match set is updated (line 17), node status flags are updated (line 18), $n$ is added to the found match for set (line 20) and then updated to the highest degree node that is unmatched (line 21) and the algorithm repeats this process.

The FindMaxDegreeNode() function initially accepts a graph (line 1) and iterates through all of the nodes which the graph contains (line 6). It calculates the degree of the current node (line 7) and compares it against the known maximum degree (line 8). If the degree of the node is larger than the current best the algorithm will update the max degree and match (lines $19 \& 20$ ), however, if the degree is equal to the known max then the algorithm switches to the cases based on the value returned from $\operatorname{rand}() \% 2$ (line 10), or a random number modulo 2. If the value returned is 0 then the algorithm takes no action (line 11), otherwise the algorithm will update the match value (line 15).

The FindMinCostNode() function initially accepts two graphs as the input (line 1), where the second graph $G_{B}$ is specifically the node and its edges to be mapped in $G_{A}$. The initial cost of $G_{B}\left(V^{\prime}\right)$ is calculated from the nodes degree $x$ (line 6). For each state in $G_{A}$ the absolute value of the cost between $G_{B}\left(V^{\prime}\right)$ these two is calculated (line 8), where the absolute value is used for events where $G_{B}\left(V^{\prime}\right)$ is larger than $G_{A}(V)$ and requires the additions of edges. If this calculated cost is less than or equal to the known minimum cost (line 9) then the algorithm will check if the two numbers are equal. This portion 


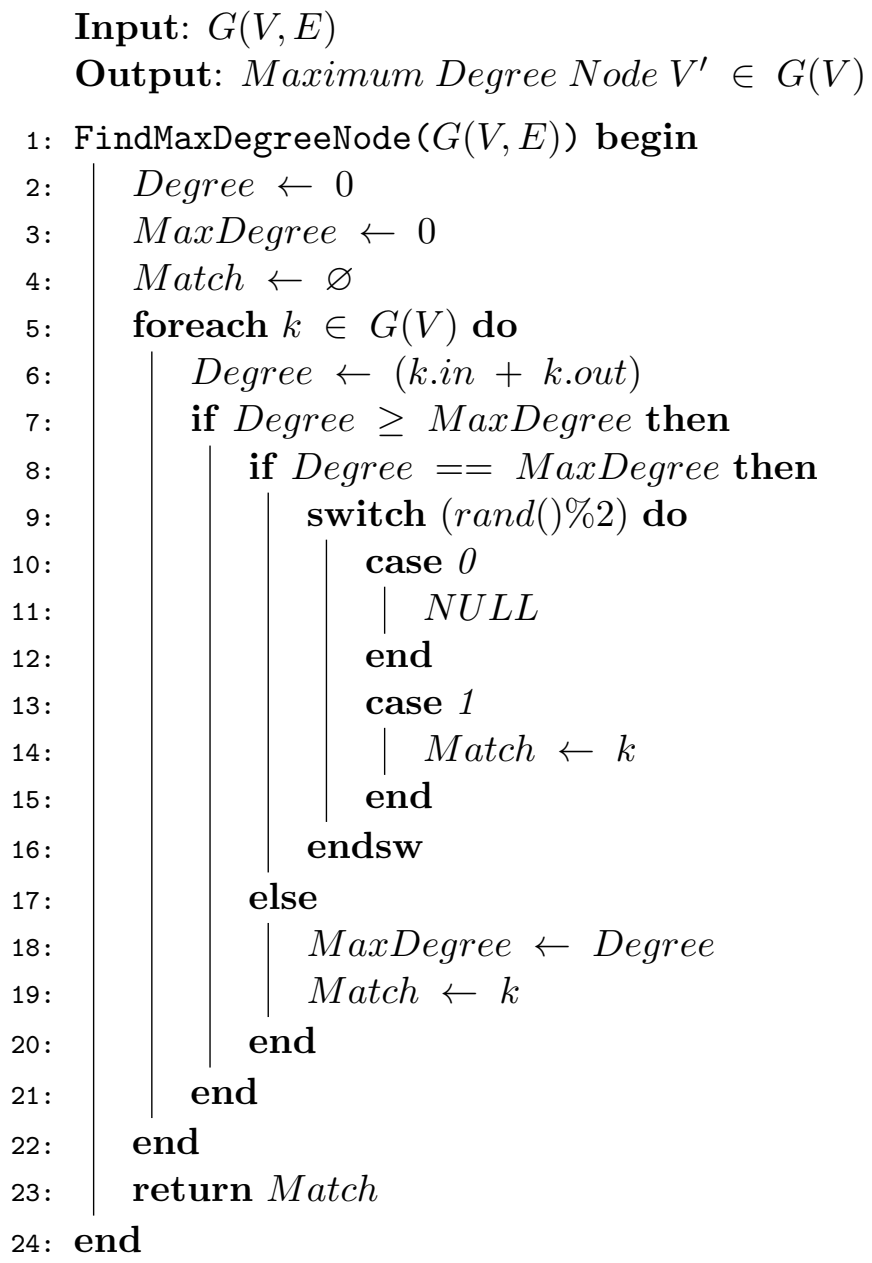

Figure 27: Algorithm for FindMaxDegreeNode function

of the algorithm operates the in the same manner for randomly selecting nodes as the FindMaxDegreeNode() function.

The Neighbors() function accepts a graph and the node for which the neighborhood is desired (line 1). The algorithm checks the set of nodes in the graph minus the center of the neighborhood (line 4). If there is a node in the graph that is also in the sub-graph of $X$, i.e., it is connected to $X$ by an incoming or outgoing edge, then this edge is added to the Neighborhood set (lines $5 \& 6$ ). Once the algorithm has examined all nodes not in the neighborhood, the algorithm returns the set of nodes connected to $X$ (line 9).

The SortDescend() function utilizes the $\mathrm{C}++$ sorting function and employs the use of a custom compare function for returning if the degree of node $k$ is larger than the degree 


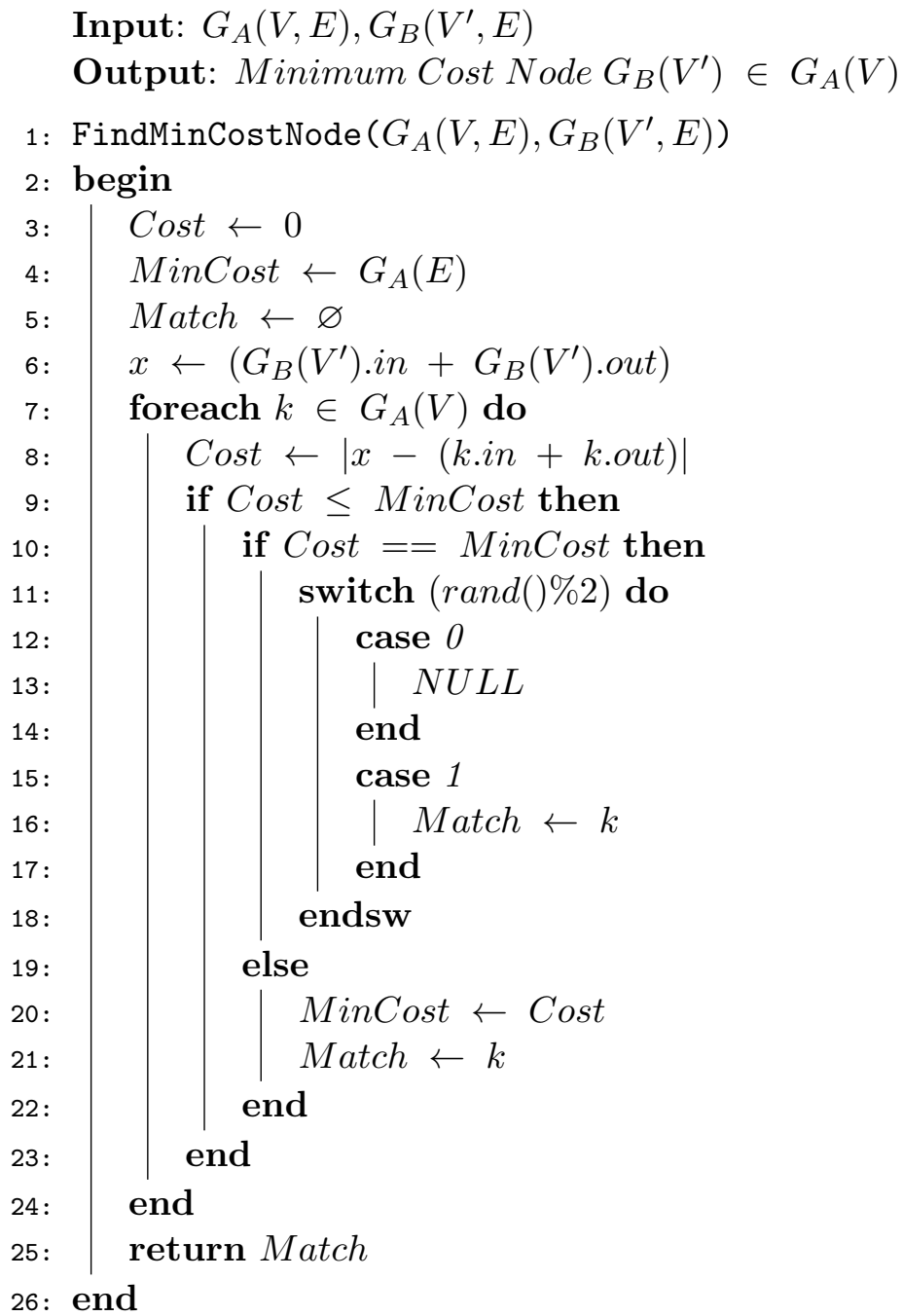

Figure 28: Algorithm for FindMinCostNode function

of node $j$. This process is repeated for the entire match set and upon completion will return the match set sorted where the node elements are in descending order of their degrees.

We illustrate the proposed greedy approach with an example where the original and watermark FSMs are shown by Fig. 31(a) and Fig. 31(b). For the sake of clarity, the example will be deterministic in the sense that we will simply choose nodes in a First Come First Serve (FCFS) manner rather than the random selection employed by the FindMaxDegreeNode() and FindMinCostNode() functions. The associated node degree values for both the original and watermark FSMs, $G_{F S M}$ and $G_{W}$, are given in Table 9. Additionally, since the the complexities behind this process as significantly less than those 


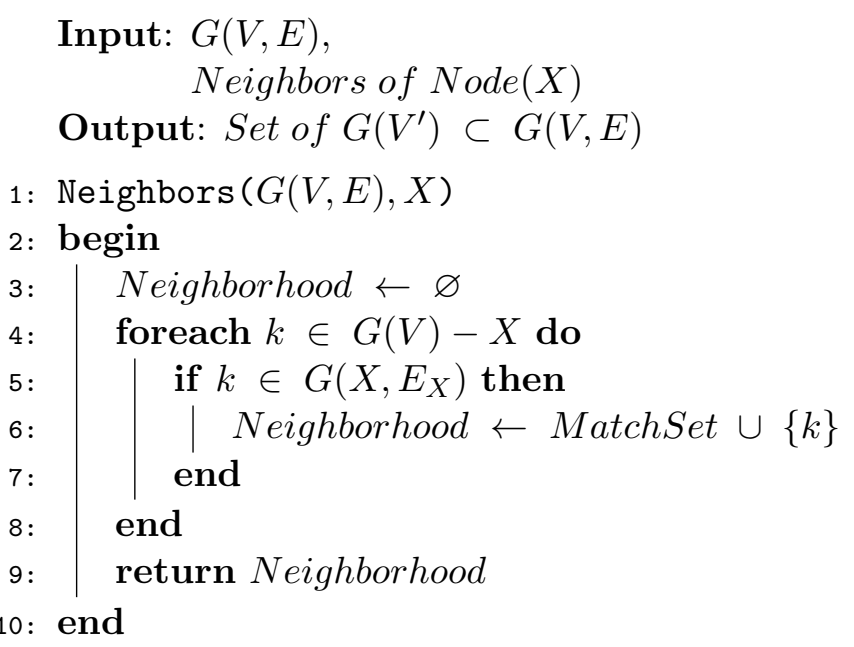

Figure 29: Algorithm for Neighbors function

Input: MatchSet

Output: Sorted MatchSet

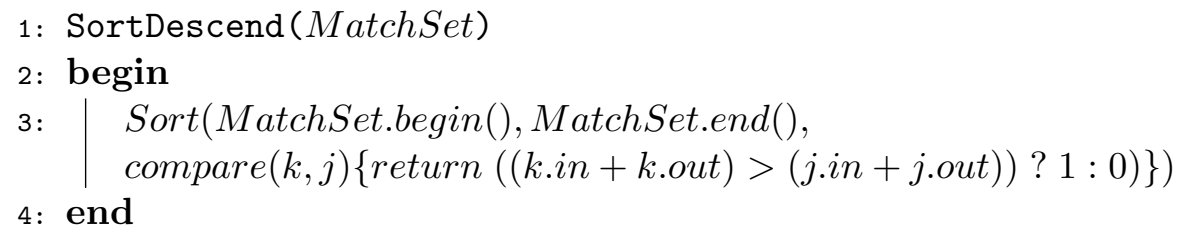

Figure 30: Algorithm for SortDescend function

of the brute force approach, the step-by-step process for which the algorithm computes the watermarked FSM is given in Table 10.

Table 9: Original and watermark FSM node degree values

\begin{tabular}{|c|c|c|c|}
\hline \multicolumn{2}{|c|}{$G_{F S M}$} & \multicolumn{2}{c|}{$G_{W}$} \\
\hline State & Degree & State & Degree \\
\hline A & 3 & 1 & 3 \\
\hline B & 4 & 2 & 3 \\
\hline C & 4 & 3 & 2 \\
\hline D & 3 & - & - \\
\hline
\end{tabular}

Upon completion of the algorithm we receive the resulting match set and additional edges are added for connection of the watermark FSM. This can be seen by Fig. 32(c), where due to the mappings additional edges are required from $2(\mathrm{C})$ to $1(\mathrm{~B})$ and $2(\mathrm{C})$ to 3(A) for the correctness of the watermark. We note that the edges with shorter, higher 


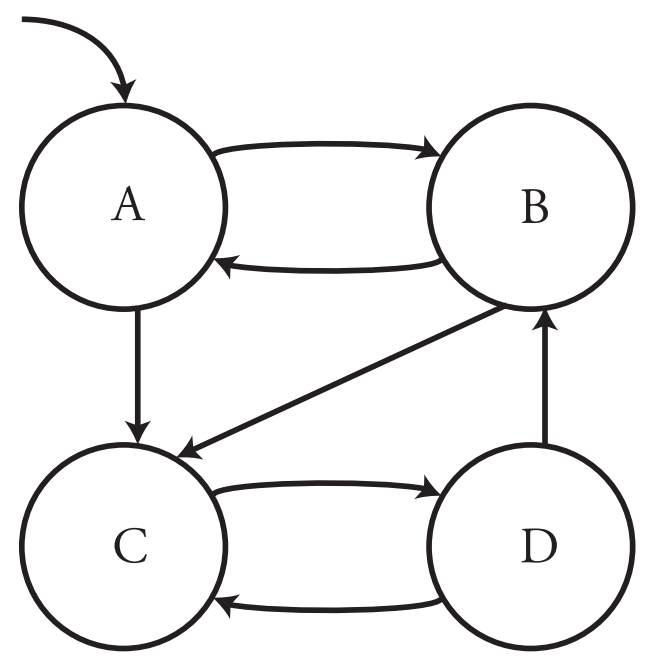

(a) Original FSM

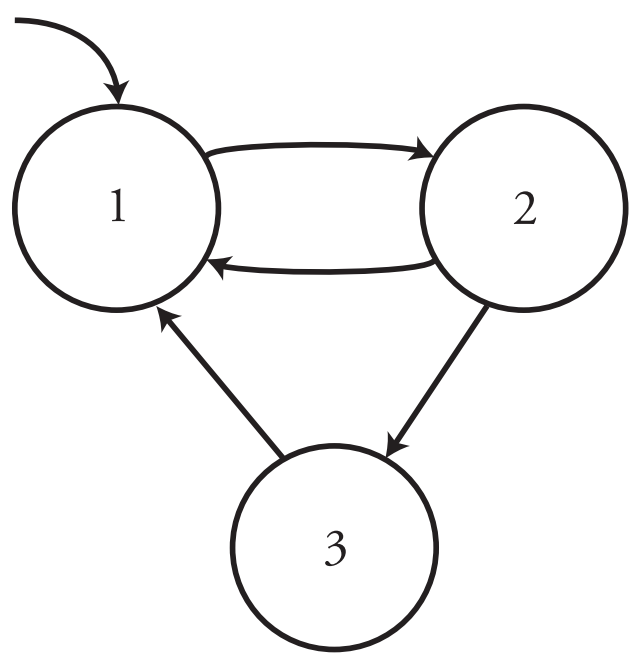

(b) Watermark FSM

Figure 31: Original and watermark FSMs for greedy embedding example

frequency, dashed lines indicated edges that overlapped in the process while the longer, lower frequency, dashed lines indicate that an edge was created. From this the cost of this solution was one edge compared to the previous perfect match from the Brute Force algorithm.

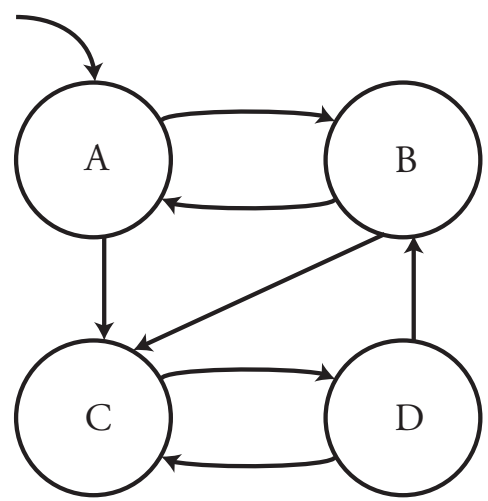

(a) Original FSM

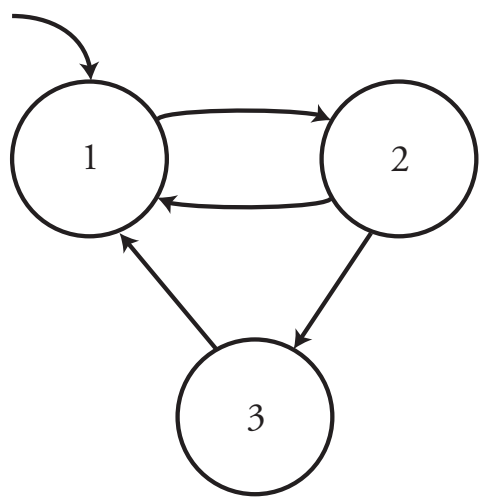

(b) Watermark FSM

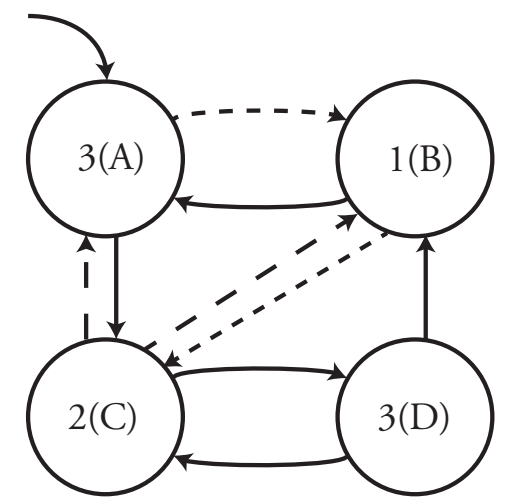

(c) Watermarked FSM

Figure 32: Original, watermark, and watermarked FSMs for greedy embedding

While a greedy solution, in addition with the HSD method, may produce more desirable results there is a need for advanced solutions. This is due to the fact that the greedy algorithm relies solely on the sparse watermark FSMs generated from the HSD method. In addition, because the implementation is greedy globally optimal solutions will 
Table 10: Example step-by-step greedy algorithm

\begin{tabular}{|c|c|c|}
\hline Step & Action & Note \\
\hline 1 & $n=B$ & FindMaxDegreeNode () returns $G_{F S M}(B)$ \\
\hline 2 & $j=1$ & FindMinCostNode () returns $G_{W}(1)$ \\
\hline 3 & MatchSet $\cup\{(B, 1)\}$ & MatchSet $\{(B, 1)\}$ \\
\hline 4 & $i=C$ & $C$ connected, unmatched, highest degree \\
\hline 5 & $k=2$ & FindMinCostNode () returns $G_{W}(2)$ \\
\hline 6 & MatchSet $\cup\{(C, 2)\}$ & MatchSet $\{(B, 1),(C, 2)\}$ \\
\hline 7 & $i=A$ & A connected, unmatched, highest degree \\
\hline 8 & $k=3$ & FindMinCostNode () returns $G_{W}(3)$ \\
\hline 9 & MatchSet $\cup\{(A, 3)\}$ & MatchSet $\{(B, 1),(C, 2),(A, 3)\}$ \\
\hline 10 & Return MatchSet & All nodes in $G_{W}$ Matched \\
\hline
\end{tabular}

not be produced. Further, we analyze the costs of this algorithm with the experimental results that were gathered, which are further detailed at length in Chapter 5. Provided in Table 11 are the results produced by this algorithm for the largest ten Kiss2 benchmark files. The watermark used was that previously shown in Fig. 19 and Fig. 20.

Table 11: Embedding results, watermark (Fig. 19) has 15 states and 32 edges

\begin{tabular}{|c|c|c|c|c|c|c|}
\hline G $_{\mathbf{F S M}}$ & States & Edges & Inputs & Edges $_{\text {Added }}$ & Edges $_{\mathbf{G}}$ & Inputs Added \\
\hline bbara_bbtas & 30 & 268 & 4 & 20 & $63 \%$ & 2 \\
\hline keyb & 19 & 170 & 7 & 18 & $56 \%$ & 1 \\
\hline kirkman & 16 & 381 & 12 & 19 & $59 \%$ & 1 \\
\hline s298 & 218 & 1096 & 3 & 21 & $66 \%$ & 2 \\
\hline s820 & 25 & 232 & 18 & 16 & $50 \%$ & 2 \\
\hline s832 & 25 & 245 & 18 & 16 & $50 \%$ & 2 \\
\hline s1488 & 48 & 251 & 8 & 14 & $44 \%$ & 1 \\
\hline s1494 & 48 & 250 & 8 & 18 & $56 \%$ & 1 \\
\hline sand & 23 & 184 & 11 & 15 & $47 \%$ & 2 \\
\hline tbk & 28 & 1569 & 6 & 13 & $41 \%$ & 2 \\
\hline
\end{tabular}

As shown in the table, more than half of the machines assumed more than $50 \%$ of the watermark edges on average, and that this method is currently less than desirable due to the fact that exemplify the algorithms performance. The benchmark "s298," for example, 
contains over 1000 edges in the system, however, this current greedy solution still adds 21 of the 32 edges. While it can be argued that 1000 edges over 218 states can easily allow for this, we turn to another example, the benchmark "tbk" contains 28 states and over 1500 edges. If we were to evenly distribute these edges over 28 states then each state roughly contains 56 edges, which contains more edges than the entirety of the watermark itself. However, due to the creation of HSD and Hash-2-Kiss2 this algorithm has greatly reduced the overhead incurred from methods such as FSD. In addition, this algorithm also allows for massive scaling compared to previous implementations. However by employing a sorting method on the Min and Max arrays for determining costs of nodes, which is comparisonbased sorting, the worst case run-times of these algorithms is known to be $\mathcal{O}(n \operatorname{logn})$ [80]. Similarly, due to the nature of the algorithm, which implements Kruskal's algorithm [81], a weighted greedy algorithm for finding a minimal spanning tree in the original FSM for which the watermark can be embedded, this run-time holds. We note that the weights in this system are associated costs of mapping a watermark state to a state in the original FSM.

\subsection{Model Generation and Verification Phase}

Once the watermark has been embedded into the original STG the model generation phase begins. Using a custom tool we have developed, called "k2vhdl," STGs corresponding Very High Speed Integrated Circuit (VHSIC) HDL (VHDL-93) [82] model is automatically generated. Figure 33 shows the system flow diagram for this tool.

The tool initially accepts a Kiss2 file and the desired Xilinx encoding scheme. The tool first checks the Kiss2 file for states that have no accepting edges from states connected to the starting state. This process is performed by calling a vertex cover function from the starting state of the FSM using an adjacency matrix computed during parsing. To better illustrate this process Fig. 34 shows the pseudo-code for the VertexCover () function. Following this the trimmed FSM is then passed to another custom tool, called "k2net," which automatically generates the Pajek netlist for viewing the FSM graph. Finally, the VHDL file has the Xilinx Synthesis Technology (XST) scripts generated automatically which allow for automation of the synthesis process. This function initially accepts the pre- 


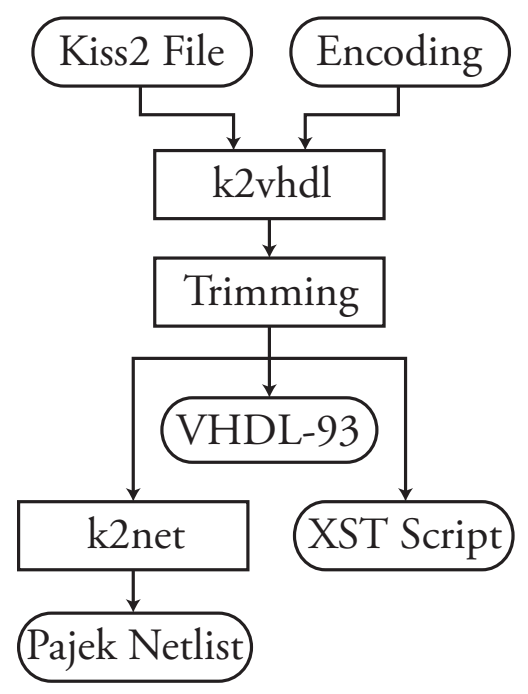

Figure 33: Flow diagram for the custom tool "k2vhdl"

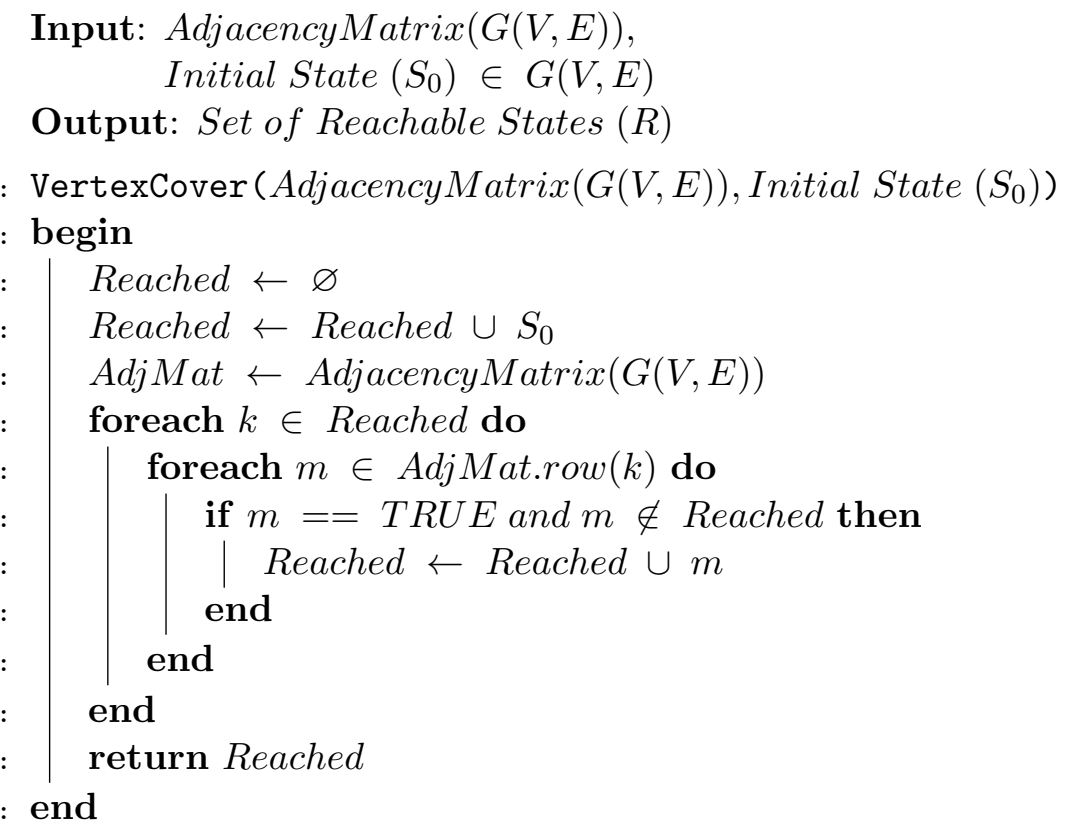

Figure 34: Algorithm for VertexCover

computed adjacency matrix for the FSM and the starting state of the system (line 1). Initially the starting state is added to the reached set (line 3) and the adjacency matrix is copied to AdjMat (line 4). On the first iteration the outer loop of the algorithm starts with $k$ as the starting state (line 5), the inner loop traverses $k$ 's adjacency matrix row (line 6) checking to see if $k$ is adjacent to $m$ and has not been reached (line 7). If $m$ is adjacent to $k$ and has not been previously reached then $m$ is added to the reached set (line 8). By adding 
adjacent nodes during the inner loop reached continues to traverse the expanded set on the following iteration. Upon completing this process the Reached set is returned (line 12). From the reached set the Kiss2 structure parsed removes the states not found in the reached set.

Through the use of Xilinx Integrated Software Environment (ISE) and target platform specific synthesis options, Xilinx ISE synthesis scripts, and Xilinx ISE project files, the synthesis results can be automatically obtained through commandline usage. During this process we are able to load, into the custom tool, an associated Xilinx synthesis script which will place the appropriate model information into the script for quick, and automated, commandline synthesis. Currently, only Xilinx VHDL compiler specific directives are generated, in the VHDL model, that allow further specifying and controlling XST options during the synthesis process. This allows for greater control of the synthesis process to prevent the watermark from being compromised at any point during synthesis.

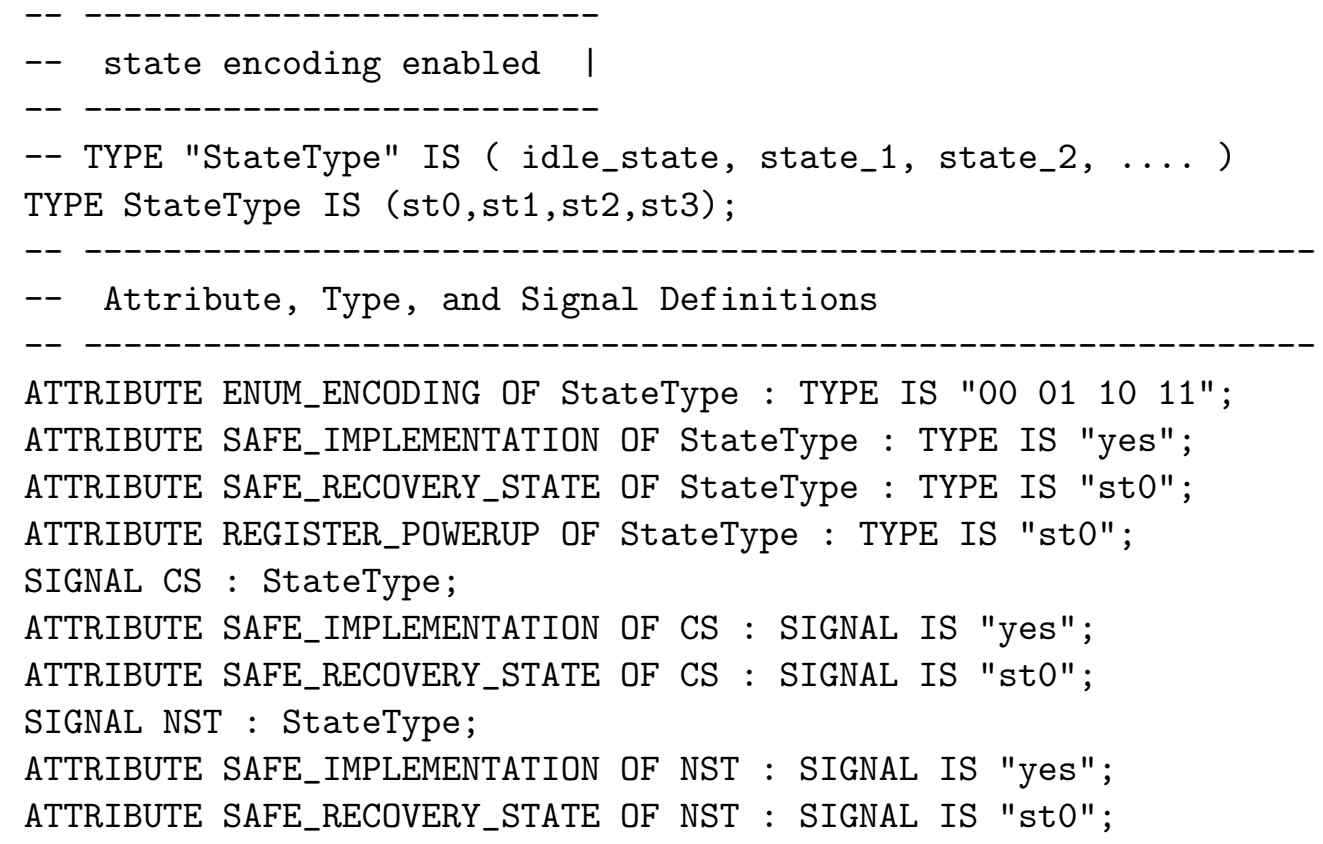

Figure 35: Sample VHDL signal generation for Lion.kiss2

Figure 35 shows an example of the VHDL-93 code that is automatically generated by the "k2vhdl" tool. The VHDL type StateType (line 5) shows how the FSM states 
are specified. Following (line 9) is example of the of the User specified state encoding allowed by Xilinx. These state encoding values, listed for the "ENUM_ENCODING" attribute, will specifically map to the states as they are listed in the body of the state machine process and for this example will specifically produce the state encoding mappings of " $\{($ st0,00), (st1,01), (st2,10), (st3,11) $\}$ ". Additionally, the attribute safe implementation is used (line 10), which specifies that in the event of an erroneous transition the state machine should go back to the safe recovery state. Lastly, the known starting state of the machine is specified, such that, the system powers up in this state (line 12).

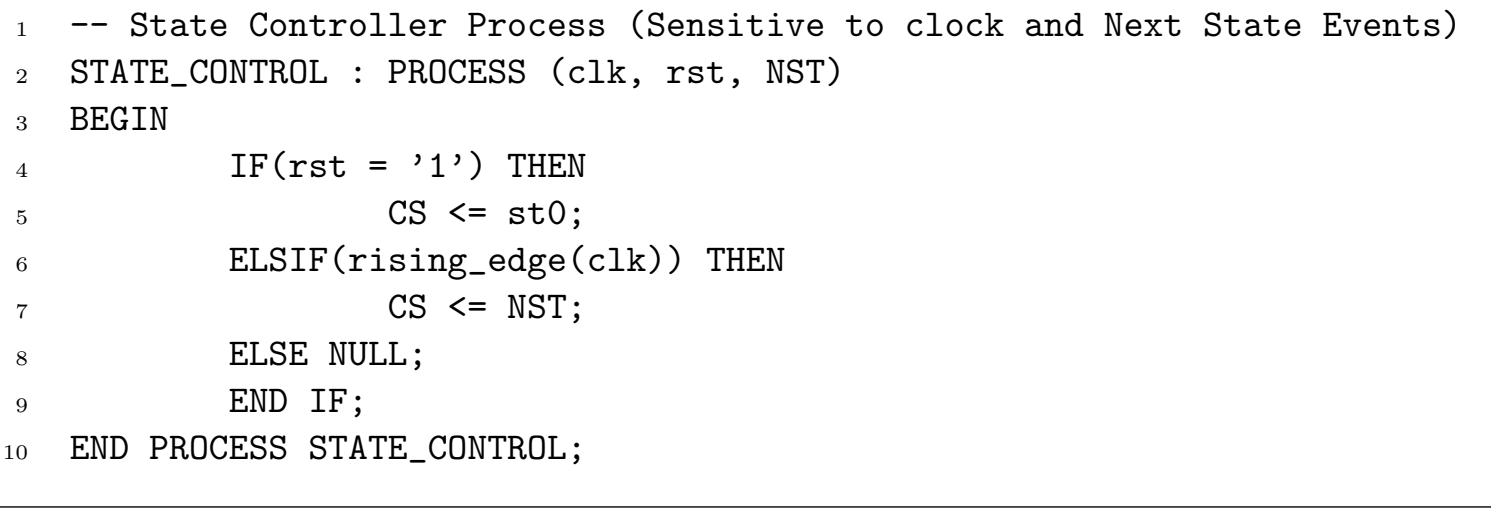

Figure 36: Sample VHDL state controller for Lion.kiss2

Figure 36 shows the process for controlling state transitions within the state machine, the process is sensitive to events on the clock, reset, and next state signals (line 2). Events in VHDL are specifically those which cause a logical transition, i.e., logical one to zero or vice verse. Additionally, the machine employs an asynchronous reset condition (line 4) while only updating the state register on a rising clock edge (line 6).

Lastly, Fig. 37 shows the state machine process itself. The state machine process is sensitive (line 2) to events on the input to the system (datai) and events on the current state (CS) which are assigned through the state control process. An output register is initialized to the length of the systems output as all zeroes (line 3). The state machine is based on a case style implementation where the current state determines the case statement which will be processed (line 5). Additionally, at each state the respective input conditions which are mapped to transitions and output functions are checked (line 6). The order in which this 


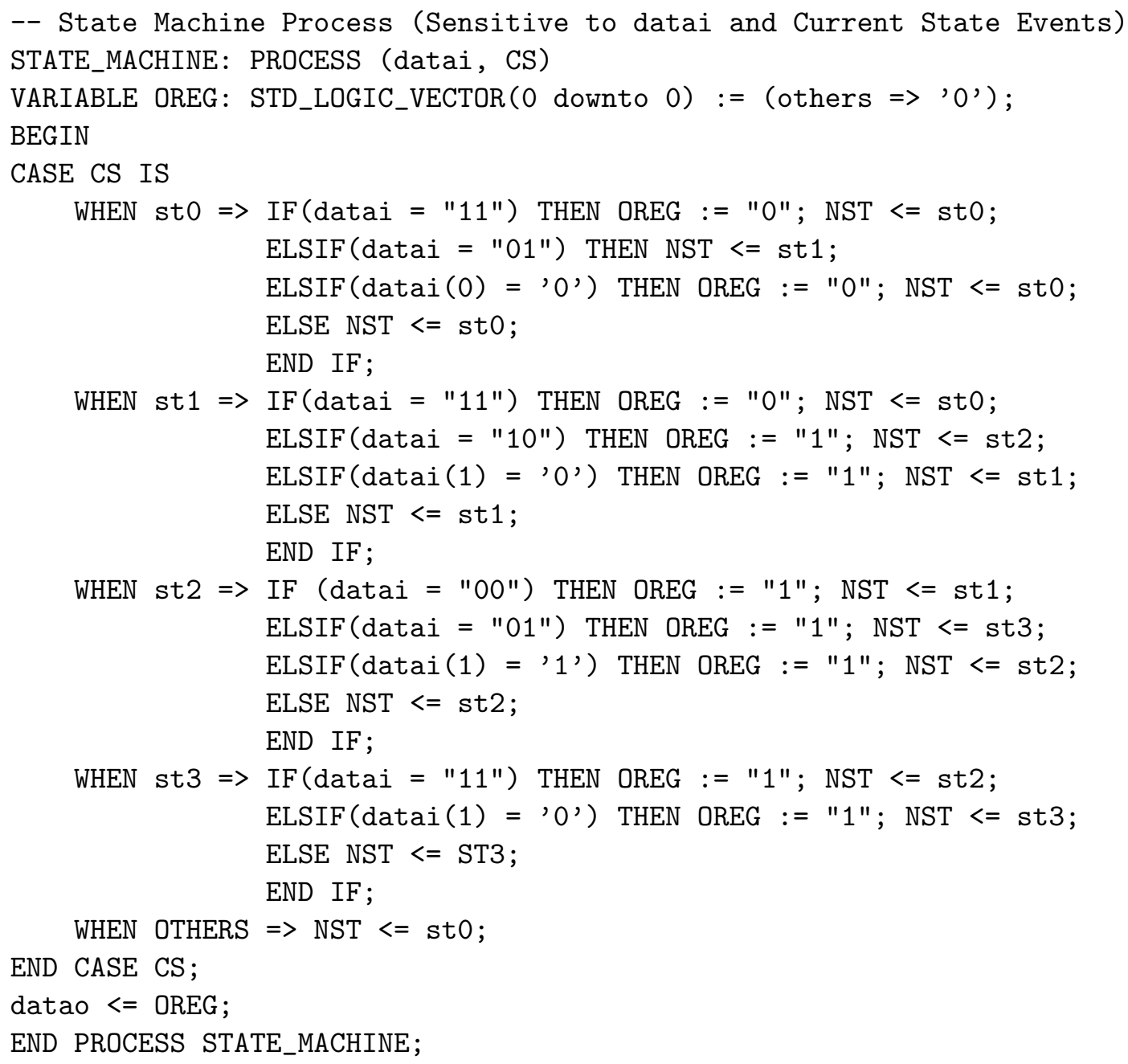

Figure 37: Sample VHDL state machine for Lion.kiss2

is done in VHDL is important, first, the completely specified input conditions are checked, then don't care conditions (lines $7 \& 8$ ). This is due to the fact that comparing input data to a don't care condition, i.e., "-0" in VHDL will always evaluate to false. Thus, the specific positions of the input bit string which will cause a transition or output are specifically checked (line 8). If there is an input not handled in the current state, the system remains in the current state and the output is not affected (line 9). If there was a condition where the output was known to be a don't care the system holds the output (line 7). In addition to system transitions, output updates are stored to a process variable to be updated once the case statement has ended. This update to the register is instantaneous rather than that 
of the signal which experiences a natural delay (line 6). Once the case statement has ended the output register is pushed to the system output port (datao) experiencing the natural propagation delay, known as delta delay, of the the system (line 31).

\subsection{Watermark Extraction Sequence Generation}

Finally once the model has been generated, we need to generate an sequence for the watermark extraction. This is automated by a tool that checks the original hash sequence against the newly created STG. In doing so it simply accepts the hash signature in state form, and finds the associated input sequence for traversing to the next hash signature state. These input pairs can either be the added dummy edges or an already specified input combination of the state. The program simply finds the first edge input combination that will take it to the next adjacent signature state. At synthesis level the watermark can easily be extracted, such that, Xilinx simulation allows you to trace the state encoding value throughout simulation. Shown in Fig. 38(a) and Fig. 38(b), is an example VHDL simulation that traces the state encoding through the simulation. Through the use of simulation state tracing embedded watermarks can easily be verified by simply applying the known sequence that will reproduce the hexadecimal hashing signature that is know represented in binary format as the state encoding.

The means of extraction during run-time or operation are generally left to the IP owner, this is simply due to the versatility of this system, such that, additional output logic could be generated automatically or the decision can be left to the IP owner and the extraction techniques for which they desire. State encoding values could be output through ports, output by seven-segment displays, stored to memory, accessed through sidechannel properties, such as power, etc. If we were to constrain the user to implement a specific system for extracting the watermark we believe it would potentially degrade the favorability of the system removing from the benefits due to large numbers of constraints required for an additional layer of protection and security. 


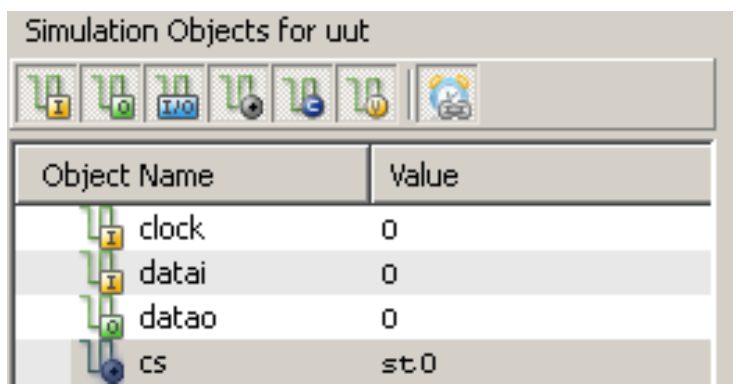

(a) State Trace "st0"

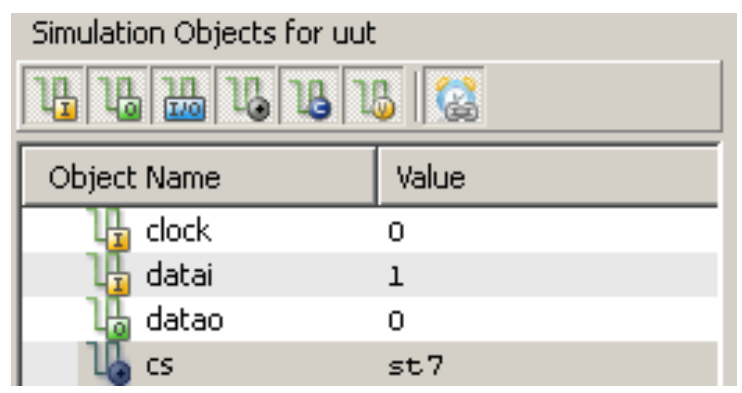

(b) State Trace "st7"

Figure 38: Xilinx simulation state encoding trace

\subsection{On the Tampering Hardness of State Encoding Based Watermarking}

In this section we perform an analysis of the tampering hardness of state encoding based watermarking. Specifically, we will focus on the complexities involved in certain scenarios, later covered in this section, that are required in tampering the watermark. We will refer to this scenario as the Bill and Mallory scenarios, where Bill represents the IP core owner and Mallory represents the malicious attacker. For this section, we make this assumption that we standardize the hashing function for this system to a hexadecimal hash sequence generated by the RIPEMD-160 hash function, such that, we have standardized both the length of the hash sequence and digest size to 40 and 160 respectively.

Mallory believes that Bill has watermarked his IP core and wishes to find the watermark Bill used. For Mallory to reverse engineer Bill's watermark Mallory must perform the following actions: (1) find any hidden functionality, (2) find all paths from all nodes, and (3) perform preimage attacks on each path. Step (1) in the process requires that Mallory formally verify the functionality of the FSM. This process requires that Mallory applies all possible input combinations for each state $\mathcal{O}\left(2^{\text {inputs }} \times\right.$ states $)$ to expose any hidden functionality. In the event that Mallory exposes hidden functionality then Mallory must compute all paths of length 40 (the length of the RIPEMD-160 hash sequence) starting at every in the FSM. The number of paths can be computed through the connectivity matrix of the FSM [83]. The connectivity matrix is built by multiplying the FSM adjacency matrix by itself $x$ times, where $x$ is the length of the desired path. Assuming Mallory carries out matrix multiplication in $\mathcal{O}\left(n^{3}\right)$ time, where $n$ is the size of the $n \times n$ adjacency matrix, and 
this process must be performed 40 times. Once Mallory has generated all paths, she must perform a preimage attack on each path. A preimage attack is where an attacker attempts to find the original file $m$ for a hash sequence $h$ that was generated by the hashing function $H$ [84]. The complexity of this process is determined by the digest size of the hashing function and in the case of RIPEMD-160 is $\mathcal{O}\left(2^{160}\right)$. This is now the complexity required to attack a single path that was generated, and $\mathcal{O}\left(P \times 2^{160}\right)$ represents the complexity for attacking all paths in the system.

Recent advances in High-Performance Computing (HPC) [85] show that systems can attempt 63 billion brute force attacks on the SHA-1 hash function per second. The SHA-1 hash is similar to the RIPEMD-160 algorithm, only in that both algorithms implement the use of a digest of 160-bits. If we neglect the finer details of these hashing functions, and their complexities, we can assume that 63 billion attempts per second on a RIPEMD-160 hash sequence. Additionally, we neglect the collision resistances of both algorithms, as SHA-1 has been shown to have collisions [86] while the RIPEMD-160 hash has been shown to be collision free [72]. We examine the situation of performing exhaustive preimage attacks under the assumption that this HPC cluster [85] can attempt the same number of attempts on a hashing function with the same digest (RIPEMD-160). It is known that the preimage complexity for both SHA-1 and RIPEMD-160 is $2^{160}$, or $1.5 \times 10^{48}$, based on the digest size used. We also know that the HPC cluster make 63 billion, or $6.3 \times 10^{10}$, attempts on SHA-1 per second. We can represent the time it takes for this system to complete a preimage attack by equation 6 . Where the number of unchecked sequences $U$ in the preimage attack is the total number of sequences minus the number of attempts per second. We desire the point where $s$ equates $U$ to being zero, such as the attack has completed, which is $s=2.3 \times 10^{37}$ seconds. Relating this number to functions of time Table 12 shows that even with HPC systems a preimage attack on this system is still computationally infeasible.

$$
\operatorname{Unchecked}_{\text {Sequences }}(U)=\left(1.5 \times 10^{48}\right)-\left(\left(6.3 \times 10^{10}\right) s\right)
$$


Table 12: Time for HPC preimage attacks

\begin{tabular}{|c|c|c|}
\hline Time Unit & Equation & Value \\
\hline \hline Seconds & $s_{\text {Seconds }}=\frac{1.5 \times 10^{48}}{6.3 \times 10^{10}}$ & $2 \times 10^{37}$ \\
\hline Minutes & $s_{\text {Minutes }}=\frac{s_{\text {Seconds }}}{60}$ & $4 \times 10^{35}$ \\
\hline Hours & $s_{\text {Hours }}=\frac{s_{\text {Minutes }}}{60}$ & $7 \times 10^{33}$ \\
\hline Days & $s_{\text {Days }}=\frac{s_{\text {Hours }}}{24}$ & $3 \times 10^{32}$ \\
\hline Weeks & $s_{\text {Weeks }}=\frac{s_{\text {Days }}}{7}$ & $4 \times 10^{31}$ \\
\hline Years & $s_{\text {Years }}=\frac{s_{\text {Weeks }}}{52}$ & $8 \times 10^{29}$ \\
\hline Decades & $s_{\text {Decades }}=\frac{s_{\text {Years }}}{10}$ & $8 \times 10^{28}$ \\
\hline Centuries & $s_{\text {Centuries }}=\frac{s_{\text {Decades }}}{10}$ & $8 \times 10^{27}$ \\
\hline Millenniums & $s_{\text {Millenniums }}=\frac{s_{\text {Centuries }}}{10}$ & $8 \times 10^{26}$ \\
\hline
\end{tabular}

Alternatively, consider the scenario where Mallory discovers Bill's signature and corresponding hash sequence. Mallory now wishes to find an alternative signature to Bill's which will also produce the same hash sequence for the ability to claim false ownership of Bill's IP core. This type of attack is known as a secondary preimage attack and is known to be computationally equivalent to a preimage attack [84]. Thus, both attacks are known to be computationally infeasible.

Lastly, consider the scenario where Bill publicly discloses the signature and corresponding hash sequence embedded. Mallory now wishes to use Bill's signature find another signature which will produce the same hash sequence. This is what is known as a collision attack and is where an attacker attempts to find a pair or signatures which will produce the same hash sequence [84]. Collision resistance is defined as $2^{80}$, or $1.2 \times 10^{24}$, for hash functions using a 160-bit digest which is due to probabilities of random selection for data blocks in this type of attack [84]. From this we can modify equation 6 to equation 7 and solve for $s$. Table 13 shows that even though the complexity has been cut in half, the time taken to perform a collision based attack is still computationally infeasible.

$$
\text { Unchecked }_{\text {Sequences }}(U)=\left(1.2 \times 10^{24}\right)-\left(\left(6.3 \times 10^{10}\right) s\right)
$$


Table 13: Time for HPC collision attacks

\begin{tabular}{|c|c|c|}
\hline Time Unit & Equation & Value \\
\hline \hline Seconds & $s_{\text {Seconds }}=\frac{1.2 \times 10^{24}}{6.3 \times 10^{10}}$ & $2 \times 10^{13}$ \\
\hline Minutes & $s_{\text {Minutes }}=\frac{s_{\text {Seconds }}}{60}$ & $3 \times 10^{11}$ \\
\hline Hours & $s_{\text {Hours }}=\frac{s_{\text {Minutes }}}{60}$ & $5 \times 10^{9}$ \\
\hline Days & $s_{\text {Days }}=\frac{s_{\text {Hours }}}{24}$ & $2 \times 10^{8}$ \\
\hline Weeks & $s_{\text {Weeks }}=\frac{s_{\text {Days }}}{7}$ & $3 \times 10^{7}$ \\
\hline Years & $s_{\text {Years }}=\frac{s_{\text {Weeks }}}{52}$ & $6 \times 10^{5}$ \\
\hline Decades & $s_{\text {Decades }}=\frac{s_{\text {Years }}}{10}$ & $6 \times 10^{4}$ \\
\hline Centuries & $s_{\text {Centuries }}=\frac{s_{\text {Decades }}}{10}$ & $6 \times 10^{3}$ \\
\hline Millenniums & $s_{\text {Millenniums }}=\frac{s_{\text {Centuries }}}{10}$ & $6 \times 10^{2}$ \\
\hline
\end{tabular}

\subsection{Chapter Summary}

Table 14 summarizes the three watermark construction methods, it is shown that the HSD watermark construction method is the best possible implementation. While both BSD and FSD have better time complexity, they lack the overall flexibility and low overhead that can be observed from the sparse watermark FSMs generated by HSD.

Table 14: Summary of proposed watermark construction phase methods

\begin{tabular}{|c|c|c|}
\hline Method & Advantages & Disadvantages \\
\hline \hline \multirow{4}{*}{ BSD } & Flexible for bitmaps & Requires simple bitmaps \\
\cline { 2 - 3 } & Sparse watermarks & Lacks flexibility in signatures \\
\cline { 2 - 3 } & Low complexity $\mathcal{O}(n \times m)$ & \\
\cline { 2 - 3 } & Low overhead & Requires files $<1 \mathrm{~KB}$ \\
\hline \hline \multirow{2}{*}{ FSD } & Flexibility in signature & Completely connected FSMs \\
\cline { 2 - 3 } & Low complexity $\mathcal{O}(n \times m)$ & Ligher complexity $\mathcal{O}\left(n^{2}\right)$ \\
\cline { 2 - 3 } & & \\
\hline \hline \multirow{3}{*}{ HSD } & Extremely flexible & \\
\cline { 2 - 3 } & Secure collision free algorithm & \\
\cline { 2 - 3 } & shortest collision free hash sequence & \\
\cline { 2 - 3 } & Sparse watermark FSMs & \\
\cline { 2 - 3 } & Lowest overhead & \\
\cline { 2 - 3 } & Signature can be any possible format & \\
\hline
\end{tabular}


Table 15 tabulates the advantages and disadvantages of the algorithms proposed for the watermark embedding phase of this system. While the greedy approach provides the desired scalability and complexity, it typically returns solutions that are not globally optimal thus incurring higher cost. Conversely, the globally optimal solutions produced from the brute force algorithm are ideal, but the system is not scalable for complex FSMs and incurs extreme run-time complexities. We summarize the advantages and disadvantages of the

Table 15: Summary of proposed watermark embedding phase methods

\begin{tabular}{|c|c|c|}
\hline Method & Advantages & Disadvantages \\
\hline \hline \multirow{2}{*}{ Brute Force } & Ideal for small FSMs & $\mathcal{O}\left({ }^{n} P_{m}\right)$ complexity \\
\cline { 2 - 3 } & Best possible matching & Lacks scalability for complex systems \\
\hline \hline \multirow{2}{*}{ Greedy } & Scalability for complex systems & Non-optimal solutions \\
\cline { 2 - 3 } & $\mathcal{O}(n \log n)$ complexity & Higher cost implementations \\
\hline
\end{tabular}

methods proposed in model generation and verification phase of this system. From the information provided in Table 16, it can be seen that these methods are advantageous in this system but are typically limited by the capabilities of the software for which their use relies. For example, by using the Pajek netlist format limits the functionalities that can be taken advantage that are offered by Gephi [87]. Conversely, because the visualization tool Gephi is still a beta version tool, support of multi-graphs (Mealy Model) FSMs is limited. This does not allow for complete visualization of a FSM with multiple edges from a current state to the same next sate.

Additionally, the Hash-2-Kiss2 algorithm is currently limited to only hexadecimal hash signatures. Extraction techniques are very advantageous and the watermark is easily verified post embedding. Additionally, the post-synthesis method implemented is extremely flexible and can be altered to the IP owner's choice for extracting the watermark.

Table 17 summarizes the computation complexities involved with specific attacks that can be performed on this watermarking method. Additionally, the run-time complexities involved in attacks past finding all paths of length 40 in the FSM were shown to be computationally infeasible. 
Table 16: Summary of proposed model generation and verification methods

\begin{tabular}{|c|c|c|}
\hline Method & Advantages & Disadvantages \\
\hline \hline Hash-2-Kiss2 & Low complexity $\mathcal{O}\left(n^{2}\right)$ & Limited to hexadecimal hashes \\
\hline \hline \multirow{2}{*}{ k2net } & Low complexity $\mathcal{O}(n)$ & Limited by the format \& software \\
\hline \hline \multirow{7}{*}{ Extraction } & Proper VHDL-93 syntax & Limited to Xilinx ISE \\
\cline { 2 - 3 } & Handles don't cares correctly & \\
\cline { 2 - 3 } & Signal encoding flexibility & \\
\cline { 2 - 3 } & Properly generates XST scripts & \\
\cline { 2 - 3 } & Watermark easily verified & \\
\cline { 2 - 3 } & Watermark easily extracted & \\
\hline \hline
\end{tabular}

Table 17: Summary of security run-time analysis

\begin{tabular}{|c|c|}
\hline Attack & Run-Time \\
\hline Data-Mining Hidden Functionality & $\mathcal{O}\left(2^{\text {inputs }} \times\right.$ states $)$ \\
\hline All Paths in the FSM $X$ & $\mathcal{O}\left(n^{3}\right)$ \\
\hline Pre-Image Single Path & $\mathcal{O}\left(2^{160}\right)$ \\
\hline Pre-Image All Paths & $\mathcal{O}\left(X \times 2^{160}\right)$ \\
\hline Secondary Pre-Image Single Path & $\mathcal{O}\left(2^{160}\right)$ \\
\hline Secondary Pre-Image All Paths & $\mathcal{O}\left(X \times 2^{160}\right)$ \\
\hline Collision Single Path & $\mathcal{O}\left(2^{80}\right)$ \\
\hline Collision All Paths & $\mathcal{O}\left(X \times 2^{80}\right)$ \\
\hline
\end{tabular}


5 Experimental Results

\subsection{Note to Reader}

Portions of this chapter have been previously published (Lewandowski et al., 2012)[45] and are utilized with permission of the publisher.

\subsection{Xilinx Synthesis Options}

All of our experiments are done with Xilinx ISE version 13.2, the synthesis options specified are for the Xilinx Virtex5 FPGA (XUPV5-LX110T-F1136). Using the custom k2vhdl tool, the synthesis options shown in Fig. 39 are inserted in the generated VHDL-93. We performed simulations and obtained data for all benchmarks under the same synthesis settings, which are listed in the VHDL code shown by Fig. 39. In the remainder of this section explain synthesis options exercised.

Xilinx ISE offers a plethora of optimization options throughout the design process. Table 18 shows the area and power optimization options. Further, more detailed explanations of these synthesis options can be found in $[88,89]$.

Table 18: Xilinx XST optimization options

\begin{tabular}{|c|c|c|}
\hline XST Option & XST Value & Purpose \\
\hline OPTIMIZE & AREA & Global Optimization $\{$ Combinatorial Logic $\}$ \\
\hline OPTIMIZE_PRIMITIVES & YES & Global Optimization $\{$ Primitives $\}$ \\
\hline OPT_MODE & AREA & Global Optimization Strategy $\{$ Area $\}$ \\
\hline OPT_LEVEL & 2 & High Level Optimization $\{$ Area:Speed $\}$ \\
\hline POWER & YES & Global Optimization $\{$ Power $\}$ \\
\hline PWR_MODE & LOW & Power Optimization $\{$ Macrocells $\}$ \\
\hline NOREDUCE & {$[$ Signal $]$} & Prevents Minimization of Signal Logic \\
\hline
\end{tabular}

Additionally, as to not compromise the signature that was embedded the watermarked FSMs were constrained to prevent to reduction of logic which update the output 


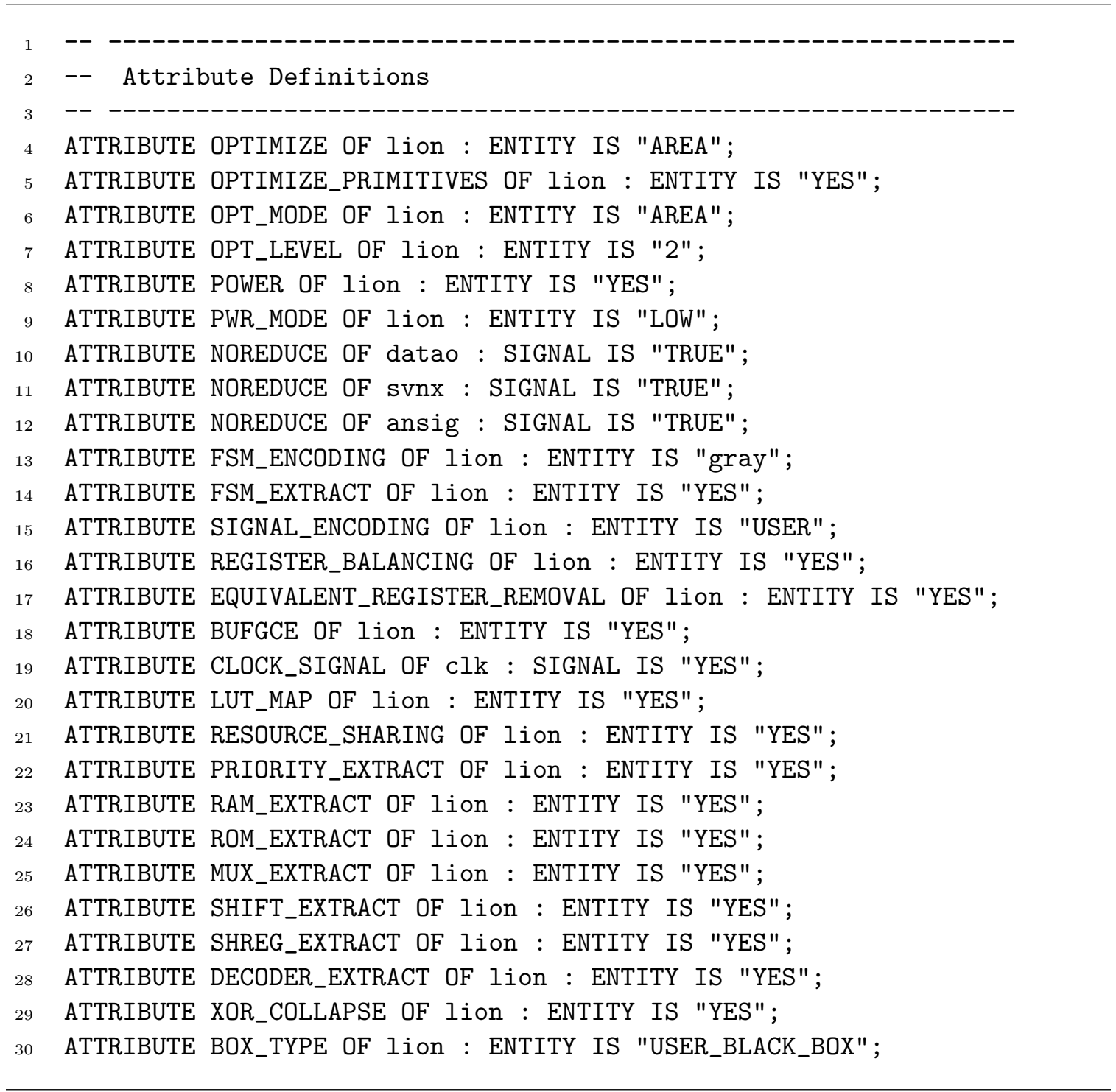

Figure 39: Sample VHDL synthesis options generation for Lion.kiss2

signal. Biased results have the potential to occur by only minimizing one FSM. From unfair minimization practices the overhead of an implementation would no longer accurately portrayed by the results. To prevent such bias each of the benchmark files used the same synthesis options which prevented the FSMs from having state reduction techniques performed. Instead of model level reduction these synthesis options were used to attempt to find optimal low-level hardware implementations for all benchmarks without compromising the signature or unfairly represent overhead. 
For the sake of clarity, we note that for "NOREDUCE" the "[Signal]" value is that of the FSM output. By implementing this synthesis constraint, XST identifies the output node of combinatorial feedback, preventing its removal, and ensuring its correct mapping [89].

\subsection{Benchmark Suite}

The benchmark suite for evaluating the proposed method is the International Workshop on Logic Synthesis (IWLS) '93 benchmark suite [90,91]. It contains roughly 50 Kiss2 format files that were developed for the use with FSM optimization tools. However, the only optimization techniques we performed were the removal of unreachable states in the machine. Table 19 shows the ten largest state FSM benchmarks after trimming unreachable states and their respective sizes.

Table 19: Top ten largest IWLS'93 Kiss2 files

\begin{tabular}{|c|c|c|}
\hline File & Number of States & Number of Edges \\
\hline bbara_bbtas & 30 & 268 \\
\hline keyb & 19 & 170 \\
\hline kirkman & 16 & 381 \\
\hline s298 & 218 & 1096 \\
\hline s820 & 25 & 232 \\
\hline s832 & 25 & 245 \\
\hline s1488 & 48 & 251 \\
\hline s1494 & 48 & 250 \\
\hline sand & 23 & 184 \\
\hline tbk & 28 & 1569 \\
\hline
\end{tabular}

Table 20, summarizes the state encoding options used in the experiments with the watermarked and original benchmark sets. In the last column we summarize the design metric optimized with the given state encoding scheme. We note that only the User encoding scheme can be employed with the watermarked FSM.

\subsection{Overhead Calculations}

In the following sections we provide details pertaining to each encoding scheme and provide the calculated overhead results. Overhead calculations are computed with 
Table 20: Encoding schemes used

\begin{tabular}{|c|c|c|c|}
\hline \multirow{2}{*}{ State Encoding } & \multicolumn{2}{|c|}{ Benchmark Set } & \multirow{2}{*}{ State Encoding Metric Notes } \\
\cline { 2 - 3 } & Original & Watermark & Stards \& Glitches \\
\hline Gray & $\mathrm{X}$ & - & Minimize Hazards \\
\hline Johnson & $\mathrm{X}$ & - & FCFS Gray \\
\hline One-Hot & $\mathrm{X}$ & - & Speed \& Power Dissipation \\
\hline Sequential & $\mathrm{X}$ & - & Minimized Next State Equations \\
\hline Speed1 & $\mathrm{X}$ & - & Speed Optimization \\
\hline User & $\mathrm{X}$ & $\mathrm{X}$ & Specified in Source by User \\
\hline
\end{tabular}

equations 8 and 9. Equations 10 and 11 are used for calculating overhead percentages for the results reported in the tables comparing the watermarked and original FSM designs.

$$
\begin{gathered}
\Delta \text { Area }_{[L U T]}\left(x^{\prime}, x\right)=\left(\sum L U T_{\left[x^{\prime}\right]}\right) /\left(\sum L U T_{[x]}\right) \\
\Delta \text { Frequency }_{[\text {Max }]}\left(x^{\prime}, x\right)=\left(\text { Frequency }_{\left[x^{\prime}\right]}\right) /\left(\text { Frequency }_{[x]}\right) \\
\% \text { Overhead }[\Delta \text { Area }]\left(x^{\prime}, x\right)=100\left[\left(\Delta \text { Area }_{[L U T]}\left(x^{\prime}, x\right)\right)-1\right] \\
\% \text { Overhead }[\Delta \text { Frequency }]\left(x^{\prime}, x\right)=100\left[\left(\Delta \text { Frequency }_{[\text {Max }]}\left(x^{\prime}, x\right)\right)-1\right]
\end{gathered}
$$

We note that, $x^{\prime}$ and $x$ represent the watermarked and original FSMs, respectively. To provide a baseline for computing these overhead calculations, original and watermarked designs are synthesized with the same synthesis options with the exception of state encoding. All results were generated using pre-generated XST synthesis scripts through Xilinx commandline usage of the Xilinx Tcl Shell.

\subsubsection{User Encoding}

Xilinx ISE Design Suite allows users to alter synthesis constraints and settings, which allows for FSMs to implement a user specified state encoding. Using this XST command allows us to enforce the specified state encoding after the watermark had been embedded into the original FSMs. This user encoding is specified as a sequential state encoding that follows the state labels from the watermarked Kiss2 file, such that, "st0" in a Kiss2 file 
has its state encoding value strictly enforced as “...000" during synthesis. This allows us to preserve the watermark state encoding and mappings created during the watermarking phase from Section 4.6. Table 21 shows the baseline synthesis results of the benchmarks. Each of the two FSMs, original and watermarked, utilize the enforced User state encoding scheme.

Table 21: Xilinx synthesis results for User \& User encoded FSMs

\begin{tabular}{|c||c|c||c|c||c|c|}
\hline \multicolumn{1}{|c||}{} & \multicolumn{2}{c||}{ Un-Watermarked } & \multicolumn{2}{c||}{ Watermarked } & \multicolumn{2}{c|}{ Overhead (\%) } \\
\hline \hline File & $\begin{array}{c}\text { Area } \\
\text { LUTs }\end{array}$ & $\begin{array}{c}\text { Frequency } \\
\text { MHz }\end{array}$ & $\begin{array}{c}\text { Area } \\
\text { LUTs }\end{array}$ & $\begin{array}{c}\text { Frequency } \\
\text { MHz }\end{array}$ & $\begin{array}{c}\text { Area } \\
\text { LUTs }\end{array}$ & $\begin{array}{c}\text { Frequency } \\
\text { MHz }\end{array}$ \\
\hline bbara_bbtas & 59 & 328 & 141 & 354 & 139 & 8 \\
\hline keyb & 120 & 348 & 114 & 313 & -5 & -10 \\
\hline kirkman & 90 & 381 & 163 & 480 & 81 & 26 \\
\hline s298 & 360 & 244 & 514 & 242 & 43 & -1 \\
\hline s820 & 90 & 409 & 384 & 260 & 327 & -36 \\
\hline s832 & 101 & 379 & 349 & 232 & 246 & -39 \\
\hline s1488 & 147 & 332 & 223 & 317 & 52 & -5 \\
\hline s1494 & 152 & 369 & 199 & 340 & 31 & -8 \\
\hline sand & 235 & 282 & 506 & 215 & 115 & -25 \\
\hline tbk & 180 & 331 & 278 & 344 & 54 & 5 \\
\hline \hline \multicolumn{2}{|c|}{} & & & Average & 108 & -9 \\
\hline
\end{tabular}

\subsubsection{Gray Encoding}

The Gray state encoding scheme operates in the same manner as binary Gray code [92], such that, given a list of binary numbers two successive numbers are separated at most by a Hamming Distance [93] of one. This is illustrated with a 2-bit example in Table 22

As illustrated, the Hamming Distance of one can be simply explained as, if two rows are adjacent then the difference in the summation of " $1 \mathrm{~s}$ " in each of the binary strings in the rows being compared is one. From this, Gray code can be used to avoid hazards and logic glitches in the system by guaranteeing that only one state variable will switch [88], 
Table 22: 2-bit Gray encoding \& Hamming distance

\begin{tabular}{|c|c|c|c|}
\hline Value & \multicolumn{3}{|c|}{ Gray Value } \\
\hline & {$[0]$} & {$[1]$} & {$[2]$} \\
\hline 0 & 0 & 0 & 0 \\
\hline 1 & 0 & 0 & 1 \\
\hline 3 & 0 & 1 & 1 \\
\hline 2 & 0 & 1 & 0 \\
\hline
\end{tabular}

maintaining a hamming distance of one, between two consecutive states. Table 23 reports the synthesis results and calculated overhead for the watermarked and original FSMs.

Table 23: Xilinx synthesis results for Gray \& User encoded FSMs

\begin{tabular}{|c||c|c||c|c||c|c|}
\hline \multicolumn{1}{|c||}{} & \multicolumn{2}{c||}{ Un-Watermarked } & \multicolumn{2}{c||}{ Watermarked } & \multicolumn{2}{c|}{ Overhead (\%) } \\
\hline \hline File & $\begin{array}{c}\text { Area } \\
\text { LUTs }\end{array}$ & $\begin{array}{c}\text { Frequency } \\
\text { MHz }\end{array}$ & $\begin{array}{c}\text { Area } \\
\text { LUTs }\end{array}$ & $\begin{array}{c}\text { Frequency } \\
\text { MHz }\end{array}$ & $\begin{array}{c}\text { Area } \\
\text { LUTs }\end{array}$ & $\begin{array}{c}\text { Frequency } \\
\text { MHz }\end{array}$ \\
\hline bbara_bbtas & 54 & 361 & 141 & 354 & 161 & -2 \\
\hline keyb & 120 & 275 & 114 & 312 & -5 & 13 \\
\hline kirkman & 72 & 379 & 163 & 480 & 126 & 26 \\
\hline s298 & 357 & 235 & 514 & 241 & 43 & 2 \\
\hline s820 & 87 & 384 & 384 & 260 & 341 & -32 \\
\hline s832 & 96 & 370 & 349 & 231 & 263 & -37 \\
\hline s1488 & 147 & 344 & 223 & 316 & 51 & -8 \\
\hline s1494 & 154 & 341 & 199 & 340 & 29 & 0 \\
\hline sand & 220 & 280 & 506 & 214 & 130 & -23 \\
\hline tbk & 189 & 317 & 278 & 343 & 47 & 8 \\
\hline \hline \multicolumn{2}{||}{} & & & Average & 119 & -5 \\
\hline
\end{tabular}

\subsubsection{Johnson Encoding}

The Johnson encoding scheme operates in a similar method to Gray, showing benefit for long paths with no branching [88]. The Johnson Encoding scheme is based off of Johnson's original algorithm for shortest paths which operated on sparse graphs [17]. Under these conditions it is most likely to return poor results for CSFSM. Using the FSM from Fig. 1, and Xilinx Synthesis, Table 24 illustrates this encoding scheme. 
Table 24: Johnson encoding

\begin{tabular}{|c|c|}
\hline State & Johnson Value \\
\hline st0 & 00 \\
\hline st1 & 01 \\
\hline st2 & 11 \\
\hline st3 & 10 \\
\hline
\end{tabular}

Table 24 shows that the Johnson encoding scheme only slightly differs from Gray code. For the Johnson encoding rather than assigning "st3" the value of "11," as the Gray encoding scheme would, the Johnson encoding scheme instead applies a Gray coding in a FCFS manner and assigns "st3" the value of "10." Table 25 reports the synthesis results and calculated overhead for the original FSMs using the Johnson encoding algorithm and the watermarked FSMs using the User enforced state encoding.

Table 25: Xilinx synthesis results for Johnson \& User encoded FSMs

\begin{tabular}{|c||c|c||c|c||c|c|}
\hline \multicolumn{1}{|c||}{} & \multicolumn{2}{c||}{ Un-Watermarked } & \multicolumn{2}{c||}{ Watermarked } & \multicolumn{2}{c|}{ Overhead (\%) } \\
\hline \hline File & $\begin{array}{c}\text { Area } \\
\text { LUTs }\end{array}$ & $\begin{array}{c}\text { Frequency } \\
\text { MHz }\end{array}$ & $\begin{array}{c}\text { Area } \\
\text { LUTs }\end{array}$ & $\begin{array}{c}\text { Frequency } \\
\text { MHz }\end{array}$ & $\begin{array}{c}\text { Area } \\
\text { LUTs }\end{array}$ & $\begin{array}{c}\text { Frequency } \\
\text { MHz }\end{array}$ \\
\hline bbara_bbtas & 214 & 279 & 141 & 354 & -34 & 26 \\
\hline keyb & 167 & 282 & 114 & 312 & -31 & 10 \\
\hline kirkman & 115 & 346 & 163 & 480 & 41 & 38 \\
\hline s298 & 5276 & 117 & 514 & 241 & -90 & 105 \\
\hline s820 & 192 & 261 & 384 & 260 & 100 & 0 \\
\hline s832 & 191 & 231 & 349 & 231 & 82 & 0 \\
\hline s1488 & 544 & 175 & 223 & 316 & -59 & 80 \\
\hline s1494 & 541 & 183 & 199 & 340 & -63 & 85 \\
\hline sand & 297 & 247 & 506 & 214 & 70 & 34 \\
\hline tbk & 564 & 196 & 278 & 343 & -50 & 74 \\
\hline \hline \multicolumn{2}{|c|}{} & & & Average & -3 & 46 \\
\hline
\end{tabular}

\subsubsection{One-hot Encoding}

One-hot state encoding is a state encoding method that ensures that each state register is dedicated solely to a single state, therefore a single register is active at a given 
time. This means that the number of registers needed is equal to the number of states in the system and the length of the encoding string will be of the same length. Table 26 shows an example of the One-hot encoding scheme.

Table 26: One-hot encoding

\begin{tabular}{|c|c|}
\hline State Value & One-Hot Value \\
\hline st0 & 1000 \\
\hline st1 & 0100 \\
\hline st2 & 0010 \\
\hline st3 & 0001 \\
\hline
\end{tabular}

Under most cases, One-hot encoding can be employed for power reduction and performance improvement [88]. Table 27 reports the synthesis results and calculated overhead for the original FSMs using the One-hot encoding algorithm and the watermarked FSMs using the User enforced state encoding.

Table 27: Xilinx synthesis results for One-hot \& User encoded FSMs

\begin{tabular}{|c||c|c||c|c||c|c|}
\hline \multicolumn{1}{|c||}{} & \multicolumn{2}{c||}{ Un-Watermarked } & \multicolumn{2}{c||}{ Watermarked } & \multicolumn{2}{c|}{ Overhead (\%) } \\
\hline \hline File & $\begin{array}{c}\text { Area } \\
\text { LUTs }\end{array}$ & $\begin{array}{c}\text { Frequency } \\
\text { MHz }\end{array}$ & $\begin{array}{c}\text { Area } \\
\text { LUTs }\end{array}$ & $\begin{array}{c}\text { Frequency } \\
\text { MHz }\end{array}$ & $\begin{array}{c}\text { Area } \\
\text { LUTs }\end{array}$ & $\begin{array}{c}\text { Frequency } \\
\text { MHz }\end{array}$ \\
\hline bbara_bbtas & 195 & 263 & 141 & 354 & -27 & 34 \\
\hline keyb & 223 & 268 & 114 & 312 & -48 & 16 \\
\hline kirkman & 167 & 219 & 163 & 480 & -2 & 118 \\
\hline s298 & 2384 & 136 & 514 & 241 & -78 & 76 \\
\hline s820 & 211 & 268 & 384 & 260 & 81 & -3 \\
\hline s832 & 226 & 245 & 349 & 231 & 54 & -5 \\
\hline s1488 & 612 & 184 & 223 & 316 & -63 & 71 \\
\hline s1494 & 626 & 158 & 199 & 340 & -68 & 114 \\
\hline sand & 373 & 191 & 506 & 214 & 35 & 73 \\
\hline tbk & 489 & 198 & 278 & 343 & -43 & 72 \\
\hline \hline \multicolumn{2}{||}{} & & & Average & -16 & 57 \\
\hline
\end{tabular}




\subsubsection{Sequential Encoding}

Sequential state encoding is the standard binary counting scheme, state-by-state and in-order, where states are assigned their appropriate binary value counterpart. This is illustrated in Table 28.

Table 28: Sequential encoding

\begin{tabular}{|c|c|}
\hline State Value & Sequential Value \\
\hline st0 & 00 \\
\hline st1 & 01 \\
\hline st2 & 10 \\
\hline st3 & 11 \\
\hline
\end{tabular}

Through the use of this state encoding systems can have their next state equations minimized [88], which will help reduce the area. Table 29 reports the synthesis results and calculated overhead for the original FSMs using the Sequential encoding algorithm and the watermarked FSMs using the User enforced state encoding.

Table 29: Xilinx synthesis results for Sequential \& User encoded FSMs

\begin{tabular}{|c||c|c||c|c||c|c|}
\hline \multicolumn{1}{|c||}{} & \multicolumn{2}{c||}{ Un-Watermarked } & \multicolumn{2}{c||}{ Watermarked } & \multicolumn{2}{c|}{ Overhead (\%) } \\
\hline \hline File & $\begin{array}{c}\text { Area } \\
\text { LUTs }\end{array}$ & $\begin{array}{c}\text { Frequency } \\
\text { MHz }\end{array}$ & $\begin{array}{c}\text { Area } \\
\text { LUTs }\end{array}$ & $\begin{array}{c}\text { Frequency } \\
\text { MHz }\end{array}$ & $\begin{array}{c}\text { Area } \\
\text { LUTs }\end{array}$ & $\begin{array}{c}\text { Frequency } \\
\text { MHz }\end{array}$ \\
\hline bbara_bbtas & 50 & 408 & 141 & 354 & 182 & -13 \\
\hline keyb & 116 & 296 & 114 & 312 & -1 & 5 \\
\hline kirkman & 90 & 380 & 163 & 480 & 81 & 26 \\
\hline s298 & 373 & 288 & 514 & 241 & 37 & -16 \\
\hline s820 & 93 & 367 & 384 & 260 & 312 & -29 \\
\hline s832 & 96 & 340 & 349 & 231 & 263 & -31 \\
\hline s1488 & 147 & 332 & 223 & 316 & 51 & -4 \\
\hline s1494 & 152 & 369 & 199 & 340 & 30 & -7 \\
\hline sand & 214 & 327 & 506 & 214 & 136 & 1 \\
\hline tbk & 201 & 316 & 278 & 343 & 38 & 8 \\
\hline \hline \multicolumn{2}{|r|}{} & & & Average & 113 & -6 \\
\hline
\end{tabular}




\subsubsection{Speed1 Encoding}

The Speed1 Encoding algorithm is designed for speed optimization [88]. The state register size is significantly increased due to the manner in which this algorithm operates, such that the number of bits used is FSM dependent. In general the Speed1 encoding scheme will assign state encoding values with a length greater than the number of states in the FSM. Using the FSM from Fig. 1, Table 30 shows this state encoding.

Table 30: Speed1 encoding

\begin{tabular}{|c|c|}
\hline State Value & Speed1 Value \\
\hline st0 & 1000 \\
\hline st1 & 0100 \\
\hline st2 & 0010 \\
\hline st3 & 0001 \\
\hline
\end{tabular}

It can be seen that, in this example, the Speed1 encoding scheme produces the same encoding scheme as One-Hot but offers a different optimization metric in doing so. Table 31 reports the synthesis results and calculated overhead for the original FSMs using the Speed1 encoding algorithm and the watermarked FSMs using the User enforced state encoding.

Table 31: Xilinx synthesis results for Speed1 \& User encoded FSMs

\begin{tabular}{|c||c|c||c|c||c|c|}
\hline \multicolumn{1}{|c||}{} & \multicolumn{2}{c||}{ Un-Watermarked } & \multicolumn{2}{c||}{ Watermarked } & \multicolumn{2}{c|}{ Overhead (\%) } \\
\hline \hline File & $\begin{array}{c}\text { Area } \\
\text { LUTs }\end{array}$ & $\begin{array}{c}\text { Frequency } \\
\text { MHz }\end{array}$ & $\begin{array}{c}\text { Area } \\
\text { LUTs }\end{array}$ & $\begin{array}{c}\text { Frequency } \\
\text { MHz }\end{array}$ & $\begin{array}{c}\text { Area } \\
\text { LUTs }\end{array}$ & $\begin{array}{c}\text { Frequency } \\
\text { MHz }\end{array}$ \\
\hline bbara_bbtas & 458 & 163 & 141 & 354 & -69 & 116 \\
\hline keyb & 250 & 231 & 114 & 312 & -54 & 35 \\
\hline kirkman & 160 & 283 & 163 & 480 & 1 & 69 \\
\hline s298 & 5669 & 117 & 514 & 241 & -90 & 105 \\
\hline s820 & 373 & 241 & 384 & 260 & 2 & 7 \\
\hline s832 & 376 & 195 & 349 & 231 & -7 & 18 \\
\hline s1488 & 693 & 178 & 223 & 316 & -67 & 77 \\
\hline s1494 & 706 & 151 & 199 & 340 & -71 & 124 \\
\hline sand & 397 & 222 & 506 & 214 & 27 & 49 \\
\hline tbk & 866 & 182 & 278 & 343 & -67 & 88 \\
\hline \hline \multicolumn{2}{|c|}{} & & & Average & -40 & 69 \\
\hline
\end{tabular}




\subsection{Discussion of Results}

We discuss the results gathered and evaluate the performance of our method. We provide a summary of the watermarked and un-watermarked findings for the six state encoding schemes. We first look at discrepancies discovered in the synthesis results.

\subsubsection{Synthesis Discrepancies}

The user enforced encoding scheme specified in the experiments follows a sequential encoding scheme. From the behavior of the k2vhdl tool, previously covered, during VHDL model generation states in the Kiss2 file are written using a sequential ordering, such that, in the body of the "state machine" VHDL process the ordering of the states within the case statement is sequential. The User encoding scheme is specified by a list of encoding values, i.e., $\{00,10,11,10\}$ will specifically map as the encoding values for the states found in the case statement. If this case statement ordering happens to be $\{s t 0, s t 3, s t 2, s t 1\}$ then the corresponding state encoding mappings will be the set of ordered pairs $\{(s t 0,00),(s t 3,10),(s t 2,11),(s t 1,10)\}$. With this knowledge, we expect the overhead comparisons between User and Sequential encoding schemes to be zero in all cases. This is based on the knowledge that Xilinx Synthesis should produce same results for the same file,

which is further supported by Xilinx guarantees that Xilinx Synthesis Technologies should be deterministic [94].

Table 32 reports the synthesis results of benchmarks using User \& Sequential state encoding. On average there was a $3 \%$ increase in LUT usage and a $1 \%$ decrease in the performance of the FSMs. Additionally, it can be seen that only 3 out of the 10 synthesis results return the $0 \%$ expected overhead.

\subsubsection{Synthesis Results}

Table 33 summarizes the results for the six state encoding schemes. We report minimum, maximum, and average overheads for area and frequency. Table 34 lists the advantages and disadvantages that this method offers with respect to the encoding schemes. 
Table 32: Xilinx synthesis discrepancies for User \& Sequential encoded FSMs

\begin{tabular}{|c||c|c||c|c||c|c|}
\hline \multicolumn{1}{|c||}{} & \multicolumn{2}{c||}{ Un-Watermarked } & \multicolumn{2}{c||}{ Un-Watermarked } & \multicolumn{2}{c|}{ Overhead $(\%)$} \\
\hline \hline File & $\begin{array}{c}\text { Area } \\
\text { LUTs }\end{array}$ & $\begin{array}{c}\text { Frequency } \\
\text { MHz }\end{array}$ & $\begin{array}{c}\text { Area } \\
\text { LUTs }\end{array}$ & $\begin{array}{c}\text { Frequency } \\
\text { MHz }\end{array}$ & $\begin{array}{c}\text { Area } \\
\text { LUTs }\end{array}$ & $\begin{array}{c}\text { Frequency } \\
\text { MHz }\end{array}$ \\
\hline bbara_bbtas & 59 & 328 & 50 & 409 & 18 & -20 \\
\hline keyb & 120 & 348 & 116 & 296 & 4 & 17 \\
\hline kirkman & 90 & 381 & 90 & 381 & 0 & 0 \\
\hline s298 & 360 & 244 & 373 & 288 & -4 & -16 \\
\hline s820 & 90 & 409 & 93 & 367 & -3 & 11 \\
\hline s832 & 101 & 379 & 96 & 340 & 5 & 11 \\
\hline s1488 & 147 & 332 & 147 & 332 & 0 & 0 \\
\hline s1494 & 152 & 369 & 152 & 369 & 0 & 0 \\
\hline sand & 235 & 282 & 214 & 327 & 10 & -14 \\
\hline tbk & 180 & 331 & 201 & 317 & -11 & 4 \\
\hline \hline \multicolumn{2}{||}{} & & & Average & 3 & -1 \\
\hline
\end{tabular}

Table 33: Summary of Xilinx synthesis results

\begin{tabular}{|c|c|c|c|c|}
\hline & \multicolumn{2}{|c|}{ Synthesis } & \multicolumn{2}{|c|}{ Overhead (\%) } \\
\hline & \multicolumn{2}{|c|}{ (Min,Max,Avg) } & \multicolumn{2}{|c|}{ (Min,Max,Avg) } \\
\hline Encoding & LUTs & Frequency & LUTs & Frequency \\
\hline \multicolumn{5}{|c|}{ Watermarked } \\
\hline User & $(114,514,287)$ & $(215,480,310)$ & $(-,-,-)$ & $(-,-,-)$ \\
\hline \multicolumn{5}{|c|}{ Un-Watermarked } \\
\hline User & $(59,360,153)$ & $(244,409,340)$ & $(-5,327,109)$ & $(-38,27,-8)$ \\
\hline Gray & $(54,357,150)$ & $(235,385,329)$ & $(-5,342,120)$ & $(-37,27,-5)$ \\
\hline Johnson & $(115,5276,810)$ & $(118,346,232)$ & $(-90,100,-4)$ & $(0,106,47)$ \\
\hline One-Hot & $(167,2384,551)$ & $(137,285,234)$ & $(-78,82,-16)$ & $(-3,119,59)$ \\
\hline Sequential & $(50,373,153)$ & $(288,409,343)$ & $(-1,313,114)$ & $(-31,27,-6)$ \\
\hline Speed1 & $(160,5669,995)$ & $(117,284,197)$ & $(-90,28,-40)$ & $(8,125,70)$ \\
\hline
\end{tabular}


Table 34: Summary of performance

\begin{tabular}{|c|c|}
\hline Advantages Against & Metrics \\
\hline \hline Johnson & Minimize Hazards \& Glitches \\
\hline One-Hot & Speed \& Power Dissipation \\
\hline Speed1 & Speed Optimization \\
\hline \hline Disadvantages Against & Metrics \\
\hline \hline User & Minimized Next State Equations \\
\hline Sequential & Minimized Next State Equations \\
\hline Gray & Minimize Hazards \& Glitches \\
\hline
\end{tabular}




\section{Future Work}

\subsection{Sequential Circuit Logic Synthesis: k3}

New methods for the advancement of this work are currently underway. These methods consist of a custom tool, dubbed "k3," that encapsulates a range of options for the minimization and optimization of FSMs. In addition, a more intuitive sequential circuit modeling language is being adapted from current, and newer, methods. Table 35 gives an overview of some of the optimization techniques, options, and support for the in-progress k3 system.

Table 35: k3 overview

\begin{tabular}{|c|}
\hline Offered Minimization Techniques \\
\hline Legacy \& New Model Support \\
\hline Trap/Dead State Removal \\
\hline Unreachable State Removal \\
\hline State Table Minimization \\
\hline Don't Care Collapsing \& Decomposition \\
\hline Clique Based Maximal Compatibility Groups \\
\hline Visualization Format File Outputs \\
\hline Legacy \& New Model Generation \\
\hline HDL \& Synthesis File Generation \\
\hline
\end{tabular}

In addition to this new system, we will explore of new methods for the embedding algorithm. As a greedy heuristic has proven to be a non-optimal, proof of concept, for embedding the watermark into the original FSM. Due to the nature of the problem and its associated complexity this can prove to be a difficult task. Currently this task is being explored through the implementation, and construction, of a simulated annealing based approach. However, while simulated annealing has a greater potential to produce desirable results, alternative algorithmic solutions are to be explored. In addition to this on-going 
work, the watermarking edge creation method is going to be further analyzed. Watermarking techniques currently available, that do not implement the use of state addition, utilize specific or unused input sequences available at a given state, we intend to explore the option of the "phantom edge," which is explained at length in the next section.

\subsection{Phantom Edges}

We define a phantom edge to be an edge in the system which does not exist, or affect the system, in normal functionality. This is achieved through the utilization of extra input bits that are otherwise non-functional, or non-system affecting, during normal operation. An example of an FSM which utilizes this technique and implements these phantom edges is shown in Fig. 40 (the phantom edges are represented by dashed lines). It can be seen that these edges are only active in a separate mode, other than the normal, in which system output is in no way affected. This absence of observable functionality allows for a better disguise of internal system behavior in the event a malicious user is observing output behavior to data mine functionality. As illustrated, the FSM utilizes modal functionality known only to the user, we also note that the bit which will cause a modal change does not have to be the least significant bit (LSB) or most significant bit (MSB) of the system, but rather, it can be inserted at any location of the input bit string and, exhibiting the same edge behavior, can achieve replication of the functionality shown.

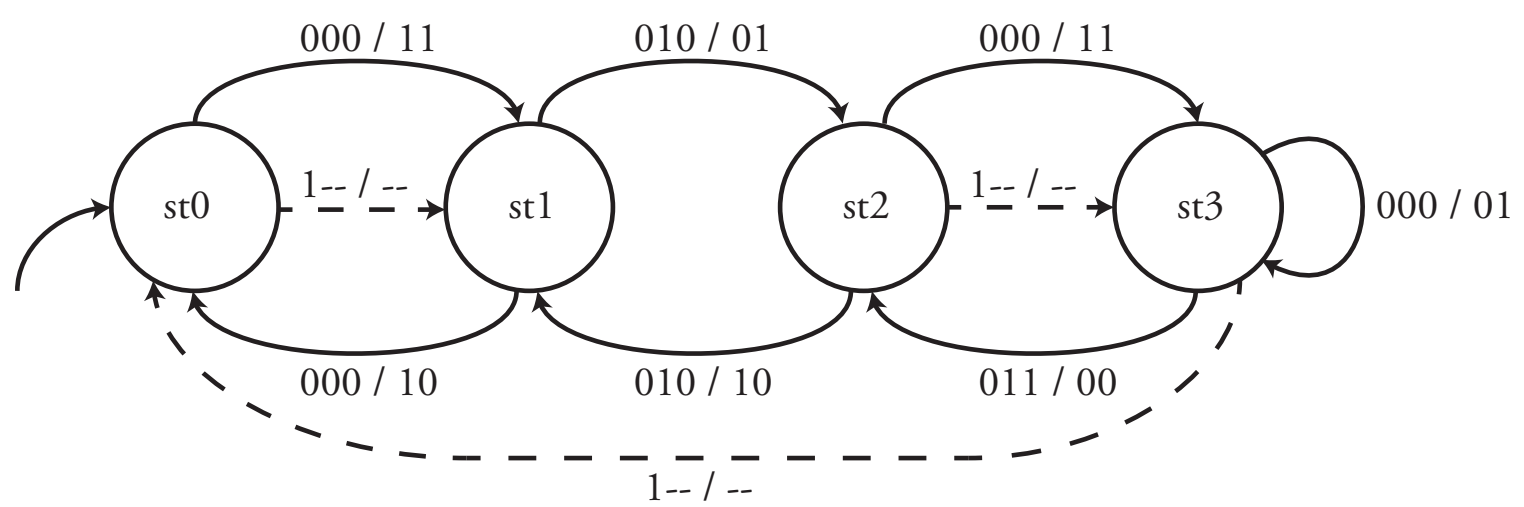

Figure 40: FSM with phantom edges

Along with phantom edge creation, this method utilizes overlapping functional edges, such that the sequence derived for recreation of the signature requires a more complex 
interaction with the system. The idea behind this is that these edges may help obfuscate functionality when an attacker attempts to formally verify all functionality.

\subsubsection{Cost of a Phantom Edge}

To further examine these phantom edges, we looked at the actual cost of the addition of a series of phantom edges through an FSM constructed using the VHDL modeling. Table 36 shows the Xilinx Synthesis results for the series of cost experiments for phantom edges.

Table 36: Xilinx synthesis results of phantom edge FSMs

\begin{tabular}{|c|c|c|c|c|c|}
\hline \multicolumn{6}{|c|}{ Fig. 40 FSM } \\
\hline \multicolumn{6}{|c|}{ Number of Phantom Edges } \\
\hline & $(0)$ & (1) & $(2)$ & $(3)$ & $(12)$ \\
\hline States & \multicolumn{5}{|c|}{4} \\
\hline Transitions & 11 & 12 & 13 & 14 & 23 \\
\hline Input Bits & 3 & \multicolumn{3}{|c|}{4} & 5 \\
\hline Output Bits & \multicolumn{5}{|c|}{4} \\
\hline Encoding & \multicolumn{5}{|c|}{ Gray } \\
\hline Implementation & \multicolumn{5}{|c|}{ LUT } \\
\hline Registers Used & \multicolumn{5}{|c|}{2} \\
\hline Look-Up Tables (LUTs) Used & \multicolumn{4}{|c|}{4} & 5 \\
\hline Max. Frequency $(\mathrm{MHz})$ & \multicolumn{5}{|c|}{1075.963} \\
\hline
\end{tabular}

It can be seen from the results in Table 36 that, based on this particular example, Phantom Edges can be expected to provide a low cost impact on area even though the number of edges in the system more than doubled. It can also been seen from this example that the addition of these edges doesn't immediately show a negative impact on the maximum frequency for which the system can operate at either.

\subsection{Watermark Extraction}

Recent exploration of side-channel watermarking from [60] employ side-channel methods for watermarking circuits. Alternative to the current watermark extraction method which requires extra means to read the state register, the exploration of the techniques em- 
ployed in this method may be beneficial for implementation within this system. In addition, this method can easily be stacked with a power profiling method and phantom edges, thus enabling the watermark to only be extracted through these hidden side-channels in the effort to prevent the system from exhibiting behavior that exposes or outputs part of the watermark.

\subsection{Metric Stacking}

The exploration of further state encoding possibilities and stacked implementations is being looked into. This is the manner of minimizing a system and strictly enforcing the state encoding beneficial to the system prior to watermarking. Conversely, embedding a signature whose watermark states are strictly enforced while the remainder of states in the FSM can have their state encoding values assigned to a given scheme for metric benefits is being explored. 


\section{Conclusions}

The proposed method of watermarking sequential circuits via state encoding is a feasible technique that can be easily employed. The proposed methods for watermark construction show that HSD is best implementation. It was shown to offer sparse watermark FSMs which lower overhead and provides the greatest flexibility allowing the signature to be a file of any format or size. Additionally, by using the RIPEMD-160 hashing function we produce the shortest secure and collision free hash sequence known.

The proposed greedy solution for watermark embedding showed to be scalable for FSMs of varying complexity and offered a significant decrease in run-time complexity. Scalability and low run-time complexity however led to higher overhead from non-optimal solutions.

Model generation and verification methods proved to be very advantageous. The "k2net" tool, for generating visualization files, provides low run-time complexity while providing a method for visualizing the Kiss2 representations of FSMs. The "Hash-2-Kiss2" tool, for generating watermark FSMs from hash sequences, proved to be a powerful tool with low-run time complexity. Most importantly, the "k2vhdl" tool proved to be an extremely powerful tool for verification, synthesis, and simulation of Kiss2 FSMs. This tool automatically generates VHDL-93 syntactically correct code, allowing for flexibility through the ability to specify state encoding schemes to be used, and reducing complications from don't care statements and unspecified state behavior.

Through the use of Xilinx simulation the watermark can easily be verified. The process of watermark extraction provides many flexible options for obtaining state encoding values at run-time. Additionally, the watermark can be easily extracted by applying the sequence used for verification. Lastly, the security of this method proves to be extensive. HPC solutions that can attempt billions of brute force attacks per second can be shown to still require a computationally infeasible amount of time. 


\section{References}

[1] R. S. Chakraborty and S. Bhunia, "HARPOON : An Obfuscation-Based SoC Design Methodology for Hardware Protection," Computer-Aided Design of Integrated Circuits and Systems, IEEE Transactions on, vol. 28, no. 10, pp. 1493-1502, 2009. [Online]. Available: http://goo.gl/eizHC

[2] A. Tirkel, G. Rankin, R. van Schyndel, W. Ho, N. Mee, and C. Osborne, "Electronic Watermark," Digital Image Computing: Techniques and Applications, 1993., 1993.

[3] F. Lancaster, "The Evolution of Electronic Publishing," Library Trends, vol. 43, no. 4, pp. 518-527, 1995.

[4] J. T. Brassil, S. Member, S. Low, N. F. Maxemchuk, and L. O. Gorman, "Electronic Marking and Identification Techniques to Discourage Document Copying," Selected Areas in Communications, IEEE Journal on, vol. 13, no. 8, pp. 1495-1504, 1995. [Online]. Available: http://goo.gl/RLDQH

[5] E. Charbon, "Hierarchical watermarking in IC design," Proceedings of the IEEE 1998 Custom Integrated Circuits Conference (Cat. No.98CH36143), pp. 295-298, 1998. [Online]. Available: http://ieeexplore.ieee.org/lpdocs/epic03/wrapper.htm?arnumber $=694985$

[6] J. Lach, W. Mangione-Smith, and M. Potkonjak, "FPGA fingerprinting techniques for protecting intellectual property," Proceedings of the IEEE 1998 Custom Integrated Circuits Conference (Cat. No.98CH36143), pp. 299-302, 1998. [Online]. Available: http://ieeexplore.ieee.org/lpdocs/epic03/wrapper.htm?arnumber=694986

[7] A. Oliveira, "Robust techniques for watermarking sequential circuit designs," Proceedings 1999 Design Automation Conference (Cat. No. 99CH36361), pp. 837-842, 1999. [Online]. Available: http://ieeexplore.ieee.org/lpdocs/epic03/wrapper.htm?arnumber $=782155$

[8] A. L. Oliveira, "Techniques for the Creation of Digital Watermarks in Sequential Circuit Designs," Computer-Aided Design of Integrated Circuits and Systems, IEEE Transactions on, vol. 20, no. 9, pp. 1101-1117, 2001. [Online]. Available: http://goo.gl/7ihp4

[9] Z. Kohavi, "Finite state machine," in Switching and Finite Automata Theory. Tata McGraw-Hill, 1998, p. 275.

[10] F. Vahid and T. Givargis, "State machine and concurrent process models," in Embedded System Design, A Unified Hardware/Software Introduction. John Wiley \& Sons, 2002, pp. $212-213$.

[11] B. D. C. Victor P. Nelson, H. Troy Nagle and J. D. Irwin, "State assignment," in Digital Logic Circuit Analysis \& Design. Prentice-Hall, 1995, p. 605. 
[12] U. Berkeley. (1992) Berkeley Logic Interchange Format (BLIF). [Online]. Available: http://embedded.eecs.berkeley.edu/Alumni/teh/research/papers/blif_spec_92.pdf

[13] E. Moore, "Gedanken-experiments on sequential machines," Automata Studies, Annals of Mathematical Studies, vol. 34, pp. 129-153, 1956.

[14] G. Mealy, "A method for synthesizing sequential circuits," The Journal of Symbolic Logic, vol. 22, no. 3, pp. 334-335, September 1957.

[15] J. L. M. Gideo Langholz, Abraham Kandel, "State assignment," in Foundations of Digital Logic Design. World Scientific, 1998, p. 385.

[16] G. Maki, I. Sawin, D.H., and B.-R. Jeng, "Improved state assignment selection tests," Computers, IEEE Transactions on, vol. C-21, no. 12, pp. 1443-1449, dec. 1972.

[17] D. B. Johnson, "Efficient algorithms for shortest paths in sparse networks," Journal of the ACM, vol. 24, no. 1, pp. 1-13, January 1977. [Online]. Available: http://dl.acm.org/citation.cfm?doid=321992.321993

[18] G. D. E. Micheli, R. Membe, and R. K. Brayton, "Optimal State Assignment for Finite State Machines," Computer-Aided Design of Integrated Circuits and Systems, IEEE Transactions on, vol. 4, no. 3, pp. 269-285, 1985. [Online]. Available: http://goo.gl/cuu4a

[19] S. Devadas, H.-K. Ma, A. Newton, and A. Sangiovanni-Vincentelli, "MUSTANG: State Assignment of Finite State Machines Targeting Multilevel Logic Implementations," Computer-Aided Design of Integrated Circuits and Systems, IEEE Transactions on, vol. 7, no. 12, pp. 1290-1300, 1988. [Online]. Available: http://ieeexplore.ieee.org/stamp/stamp.jsp?tp=\&arnumber $=16807 \&$ isnumber $=610$

[20] B. Eschermann and H.-J. Wunderlich, "Optimized synthesis of self-testable finite state machines," [1990] Digest of Papers. Fault-Tolerant Computing: 20th International Symposium, pp. 390-397, 1990. [Online]. Available: http://ieeexplore.iee.org/lpdocs/epic03/wrapper.htm?arnumber=89393

[21] S. Robinson and J. Shen, "Evaluation and synthesis of self-monitoring state machines," 1990 IEEE International Conference on Computer-Aided Design. Digest of Technical Papers, pp. 276-279, 1990. [Online]. Available: http://ieeexplore.ieee.org/lpdocs/epic03/wrapper.htm?arnumber=129901

[22] D. J. Rosenkrantz, "Half-Hot State Assignments for Finite State Machines," Computers, IEEE Transactions on, vol. 39, no. 5, pp. 700-702, 1990. [Online]. Available: http://goo.gl/dgxLY

[23] M. Perkowski and L. Nguyen, "The encoding program for concurrent finite state machines realized using PLD devices," Proceedings of the 33rd Midwest Symposium on Circuits and Systems, pp. 204-207, 1990. [Online]. Available: http://ieeexplore.ieee.org/lpdocs/epic03/wrapper.htm?arnumber $=140687$

[24] J.-j. Yang, H. Shin, J.-w. Chow, and G. Star, "New State Assignment Algorithms for Finite State Machines Using Look Ahead," Custom Integrated Circuits Conference, 1991., Proceedings of the IEEE 1991, pp. 11.2/1-11.2/4, 1991. [Online]. Available: http://goo.gl/6s69G 
[25] L. Lavagno, C. Moon, R. Brayton, and A. Sangiovanni-Vincentelli, "Solving the state assignment problem for signal transition graphs," in Design Automation Conference, 1992. Proceedings., 29th ACM/IEEE, jun 1992, pp. 568-572.

[26] G. Rietsche and F. Informatik, "State Assignment for Finite State Machines Using T Flip-Flops," Design Automation Conference, 1993, with EURO-VHDL '93. Proceedings EURO-DAC '93., European, pp. 396-401, 1993. [Online]. Available: http://goo.gl/6khX8

[27] T.-a. Chu, N. Mani, and C. K. C. Leung, "A New State Assignment Technique for Asynchronous Finite State Machines," 'Design Automation of High Performance VLSI Systems', Proceedings., Third Great Lakes Symposium on, pp. 139-143, 1993. [Online]. Available: http://goo.gl/0j4H6

[28] K.-H. Wang, W.-S. Wang, T. Hwang, A. Wu, and Y.-L. Lin, "State assignment for power and area minimization," in Computer Design: VLSI in Computers and Processors, 1994. ICCD '94. Proceedings., IEEE International Conference on, oct 1994, pp. $250-254$.

[29] T.-d. Her, W. K. Tsai, F. Kurdahi, and Y. Chen, "Low-Power Driven State Assignment of Finite State Machines," Circuits and Systems, 1994. APCCAS '94., 1994 IEEE Asia-Pacific Conference on, pp. 454-459, 1994. [Online]. Available: http://goo.gl/dh494

[30] E. Olson and S. Kang, "Assignment for Low-Power FSM Synthesis Using Genetic Local Search," Custom Integrated Circuits Conference, 1994., Proceedings of the IEEE 1994, pp. 140-143, 1994. [Online]. Available: http://goo.gl/D56EQ

[31] C. Bolchini, R. Montandon, F. Sake, D. Sciuto, and P. Milano, "A State Encoding for Self-Checking Finite State Machines," Design Automation Conference, 1995. Proceedings of the ASP-DAC '95/CHDL '95/VLSI '95., IFIP International Conference on Hardware Description Languages; IFIP International Conference on Very Large Scale Integration., Asian and South Pacific, pp. 711-716, 1995. [Online]. Available: http://goo.gl/8qP7a

[32] J. Rutten and M. Berkelaar, "Improved State Assignment for Burst Mode Finite State Machines," Advanced Research in Asynchronous Circuits and Systems, 1997. Proceedings., Third International Symposium on, pp. 228-239, 1997. [Online]. Available: http://goo.gl/oAh5V

[33] I. Ahmad and R. Ul-Mustafa, "On State Assignment of Finite State Machines Using Hypercube Embedding Approach," Computer Design, 1999. (ICCD '99) International Conference on, pp. 608-613, 1999. [Online]. Available: http://goo.gl/jAqLV

[34] P. Bacchetta, L. Daldoss, D. Sciuto, and C. Silvano, "Low-Power State Assignment Techniques for Finite State Machines," Circuits and Systems, 2000. Proceedings. ISCAS 2000 Geneva. The 2000 IEEE International Symposium on, vol. 2, pp. 641-644, 2000. [Online]. Available: http://goo.gl/tgQWt

[35] L. Jozwiak and A. Slusarczyk, "A new state assignment method targeting fpga implementations," in Euromicro Conference, 2000. Proceedings of the 26th, vol. 1, 2000, pp. 50-59. 
[36] S. K. Kuusilinna, "Finite state machine encoding for VHDL synthesis," Computers and Digital Techniques, IEE Proceedings -, vol. 148, no. 1, pp. 23-30, 2001. [Online]. Available: http://goo.gl/wLACN

[37] M. Chyzy and W. Kosiski, "Evolutionary Algorithm for State Assignment of Finite State Machines," Digital System Design, 2002. Proceedings. Euromicro Symposium on, pp. 359-362, 2002. [Online]. Available: http://goo.gl/VvY8K

[38] S. Roy, "Power conscious BIST design for sequential circuits using ghost-FSM," Proceedings of the 7th International Conference on Properties and Applications of Dielectric Materials (Cat No 03CH37417) ATS-03, pp. 190-195, 2003. [Online]. Available: http://goo.gl/qV8eS

[39] G. Venkataraman, S. Reddy, and I. Pomeranz, "Gallop: genetic algorithm based low power fsm synthesis by simultaneous partitioning and state assignment," in VLSI Design, 2003. Proceedings. 16th International Conference on, jan. 2003, pp. 533-538.

[40] S. Chattopadhyay, P. Yadav, and R. Singh, "Multiplexer targeted finite state machine encoding for area and power minimization," India Annual Conference, 2004. Proceedings of the IEEE INDICON 2004. First, pp. 12-16, 2004. [Online]. Available: http://goo.gl/3nYHA

[41] B. Tawadros and R. Guindi, "State Assignment for LowLeakage Finite State-Machines," The 3rd International IEEENEWCAS Conference, 2005., pp. 115-118, 2005. [Online]. Available: http://ieeexplore.ieee.org/lpdocs/epic03/wrapper.htm?arnumber $=1496657$

[42] L. Mengibar, L. Entrena, and M. Garc, "Partitioned state encoding for low power in FPGAs," Electronics Letters, vol. 41, no. 17, pp. 18-19, 2005. [Online]. Available: http://goo.gl/E0kGx

[43] M. Damm, "State Assignment for Detecting Erroneous Transitions in Finite State Machines," 9th EUROMICRO Conference on Digital System Design (DSD'06), pp. 483-490, 2006. [Online]. Available: http://ieeexplore.ieee.org/lpdocs/epic03/wrapper.htm?arnumber=1690077

[44] Y. Lee, K. Choi, and T. Kim, "SAT-based state encoding for peak current Minimization," 2009 International SoC Design Conference (ISOCC), pp. 432-435, 2009. [Online]. Available: http://goo.gl/Z1kLE

[45] M. Lewandowski, R. Meana, M. Morrison, and S. Katkoori, "A novel method for watermarking sequential circuits," 2012 IEEE International Symposium on Hardware-Oriented Security and Trust, pp. 21-24, Jun. 2012. [Online]. Available: http://ieeexplore.ieee.org/lpdocs/epic03/wrapper.htm?arnumber $=6224313$

[46] L. Zhang and C.-h. Chang, "State Encoding Watermarking for Field Authentication of Sequential Circuit Intellectual Property," Circuits and Systems (ISCAS), 2012 IEEE International Symposium on, pp. 3013-3016, 2012. [Online]. Available: http://goo.gl/R32oH

[47] M. Karnaugh, "The map method for synthesis of combinational logic circuits," Trans. AIEE. pt I, vol. 72, no. 9, pp. 593-599, November 1953. 
[48] I. Tomnoglu and E. Charbon, "Watermarking-Based Copyright Protection of Sequential Functions," Custom Integrated Circuits, 1999. Proceedings of the IEEE 1999, pp. 35-38, 1999. [Online]. Available: http://goo.gl/dDx6Z

[49] A. Abdel-Hamid, S. Tahar, and E. M. Aboulhamid, "IP watermarking techniques: survey and comparison," in System-on-Chip for Real-Time Applications, 2003. Proceedings. The 3rd IEEE International Workshop on, June-2 July 2003, pp. 60-65.

[50] A. Abdel-Hamid, S. Tahar, and E. Aboulhamid, "A Tool for Automatic Watermarking of IP Designs," Circuits and Systems, 2004. NEWCAS 2004. The 2nd Annual IEEE Northeast Workshop on, pp. 381-384, 2004. [Online]. Available: http://goo.gl/iAoNh

[51] — "A Public-Key Watermarking Technique for IP Designs," Design, Automation and Test in Europe, pp. 330-335, 2005. [Online]. Available: http://ieeexplore.ieee.org/lpdocs/epic03/wrapper.htm?arnumber $=1395581$

[52] A. T. Abdel-Hamid, S. Tahar, and E. Aboulhamid, "Finite State Machine IP Watermarking: A Tutorial," First NASA/ESA Conference on Adaptive Hardware and Systems (AHS'06), pp. 457-464, 2006. [Online]. Available: http://goo.gl/hZUgT

[53] S. S. P. S. Nandgawe, "Intellectual Property Protection of Sequential Circuits Using Digital Watermarking," Industrial and Information Systems, First International Conference on, no. August, pp. 8-11, 2006. [Online]. Available: http://goo.gl/ibpEU

[54] A. Stoica and S. Katkoori, "'Glitch Logic" and Applications to Computing and Information Security," in Bio-inspired Learning and Intelligent Systems for Security, 2009. BLISS '09. Symposium on, August 2009, pp. 107-112.

[55] A. Cui and C.-H. Chang, "Watermarking for IP Protection through Template Substitution at Logic Synthesis Level," 2007 IEEE International Symposium on Circuits and Systems, pp. 3687-3690, May 2007. [Online]. Available: http://ieeexplore.ieee.org/lpdocs/epic03/wrapper.htm?arnumber $=4253481$

[56] M.-C. Lin, G.-R. Tsai, C.-R. Wu, and C.-H. Lin, "Watermarking Technique for HDL-based IP Module Protection," Third International Conference on Intelligent Information Hiding and Multimedia Signal Processing (IIH-MSP 2007), pp. 393-396, Nov. 2007. [Online]. Available: http://ieeexplore.ieee.org/lpdocs/epic03/wrapper.htm?arnumber $=4457732$

[57] R. S. Chakraborty and S. Bhunia, "Hardware protection and authentication through netlist level obfuscation," 2008 IEEE/ACM International Conference on Computer-Aided Design, pp. 674-677, Nov. 2008. [Online]. Available: http://ieeexplore.ieee.org/lpdocs/epic03/wrapper.htm?arnumber $=4681649$

[58] A. Abdel-Hamid and S. Tahar, "Fragile IP Watermarking Techniques," in Adaptive Hardware and Systems, 2008. AHS '08. NASA/ESA Conference on, June 2008, pp. $513-519$.

[59] F. Koushanfar and W. Marsh, "Provably Secure Obfuscation of Diverse Watermarks for Sequential Circuits," Hardware-Oriented Security and Trust (HOST), 2010 IEEE International Symposium on, pp. 42-47, 2010. [Online]. Available: http://goo.gl/67KWA 
[60] G. Becker, M. Kasper, A. Moradi, and C. Paar, "Side-channel based watermarks for integrated circuits," in Hardware-Oriented Security and Trust (HOST), 2010 IEEE International Symposium on, June 2010, pp. 30-35.

[61] A. Cui, C.-h. Chang, S. Member, and S. Tahar, "A Robust FSM Watermarking Scheme for IP Protection of Sequential Circuit Design," Computer-Aided Design of Integrated Circuits and Systems, IEEE Transactions on, vol. 30, no. 5, pp. 678-690, 2011. [Online]. Available: http://goo.gl/cxivz

[62] A. Cui, C.-H. Chang, and L. Zhang, "A hybrid watermarking scheme for sequential functions," 2011 IEEE International Symposium of Circuits and Systems (ISCAS), pp. 2333-2336, May 2011. [Online]. Available: http://ieeexplore.ieee.org/lpdocs/epic03/wrapper.htm?arnumber $=5938070$

[63] W. $\mathrm{Xu}$ and Y. Zhu, "A digital copyright protection scheme for soft-IP core based on FSMs," 2011 International Conference on Consumer Electronics, Communications and Networks (CECNet), pp. 3823-3826, Apr. 2011. [Online]. Available: http://goo.gl/2DskW

[64] N. H. E. Weste and D. M. Harris, "Placing cute logos on a chip," in CMOS VLSI Design, A Circuits and Systems Perspective. Addison-Wesley, 2011, p. 136.

[65] MOSIS. (2010) ON Semiconductor C5 Process. USC Information Sciences Institute. [Online]. Available: http://mosis.com/vendors/view/on-semiconductor/c5

[66] — (2010) Mosis faqs: Design issues. USC Information Sciences Institute. [Online]. Available: http://www.mosis.com/pages/Faqs/faq-design

[67] M. W. Davidson. (1995) Silicon Zoo. [Online]. Available: http://micro.magnet.fsu.edu/creatures/index.html

[68] J.-B. Note and E. Rannaud, "From the bitstream to the netlist," in Proceedings of the 16th international ACM/SIGDA symposium on Field programmable gate arrays, ser. FPGA '08. New York, NY, USA: ACM, 2008, pp. 264-264. [Online]. Available: http://doi.acm.org/10.1145/1344671.1344729

[69] Hackito-Ergo-Sum. (2010) FPGA Reverse-Engineering Challenge. [Online]. Available: http://www.hackitoergosum.org/2010/HES2010-sbourdeauducq-FPGA-Challenge.pdf

[70] D. Ho. (2011) Notepad++ a free source code editor. [Online]. Available: http://notepad-plus-plus.org/

[71] H. Dobbertin, A. Bosselaers, and B. Preneel, "RIPEMD-160: A Strengthened Version of RIPEMD," Proceedings of the Third International Workshop on Fast Software Encryption, pp. 71-82, 1996. [Online]. Available: http://dl.acm.org/citation.cfm?id=647931.740583

[72] F. Mendel, N. Pramstaller, C. Rechberger, and V. Rijmen, "On the collision resistance of ripemd-160," in Proceedings of the 9th international conference on Information Security, ser. ISC'06. Berlin, Heidelberg: Springer-Verlag, 2006, pp. 101-116. [Online]. Available: http://dx.doi.org/10.1007/11836810_8 
[73] OpenSSL. (1999) OpenSSL Cryptography and SSL/TLS Toolkit. [Online]. Available: http://www.openssl.org/

[74] V. Batagelj and A. Mrvar, "Pajek Program for Large Network Analysis," Connections, Journal of International Network for Social Network Analysis, vol. 21, no. 1, pp. 1-11, 1998. [Online]. Available: http://vlado.fmf.uni-lj.si/pub/networks/doc/pajek.pdf

[75] M. Bastian and S. Heymann, "Gephi : An Open Source Software for Exploring and Manipulating Networks," International AAAI Conference on Weblogs and Social Media, pp. 1-2, 2009. [Online]. Available: http://www.aaai.org/ocs/index.php/ICWSM/09/paper/view/154

[76] T. H. Cormen, C. E. Leiserson, R. L. Rivest, and C. Stein, in Introduction to Algorithms. MIT Press and McGraw-Hill, 1998.

[77] S. A. Cook, "The complexity of theorem-proving procedures," in Proceedings of the third annual ACM symposium on Theory of computing, ser. STOC 71. New York, NY, USA: ACM, 1971, pp. 151-158. [Online]. Available: http://doi.acm.org/10.1145/800157.805047

[78] S. S. Epp, "Basics of set theory," in Discrete Mathematics with Applications. Thomson, 2004, p. 260.

[79] G. D. Micheli, in Synthesis and Optimization of Digital Circuits. McGraw-Hill, 1994.

[80] R. Johnsonbaugh and M. Schaefer, in Algorithms. Pearson, 2004.

[81] J. B. Kruskal, "On the Shortest Spanning Subtree of a Graph and the Traveling Salesman Problem," in American Mathematical Society, vol. 7, no. 1, February 1956, pp. 48-50. [Online]. Available: http://www.jstor.org/stable/2033241

[82] "IEEE Standard VHDL Language Reference Manual." ANSI/IEEE Std 1076-1993, p. i, 1994.

[83] L.-E. Thorelli, "An algorithm for computing all paths in a graph," BIT Numerical Mathematics, vol. 6, no. 4, pp. 347-349, 1966. [Online]. Available: http://dx.doi.org/10.1007/BF01966095

[84] W. Stallings, "Requirements and security," in Cryptography and Network Security. Prentice Hall, 1998, pp. 335-341.

[85] J. M. Gosney, "Password Cracking HPC," in Passwordsî2 Security Conference, December 2012, pp. 1-29. [Online]. Available: http://passwords12.at.ifi.uio.no/

[86] X. Wang, Y. L. Yin, and H. Yu. (2005) Finding Collisions in the Full SHA-1. [Online]. Available: http://people.csail.mit.edu/yiqun/SHA1AttackProceedingVersion.pdf

[87] M. Bastian and S. Heymann. (2009) Comparison of Supported Graph Formats in Gephi. [Online]. Available: http://gephi.org/users/supported-graph-formats/

[88] Xilinx. $\quad(2009, \quad$ September $) \quad$ Xilinx $\quad$ Synthesis Technology (XST) User $\quad$ Guide. [Online]. Available: http://www.xilinx.com/support/documentation/sw_manuals/xilinx11/xst.pdf 
[89] —. (2009, December) Xilinx Constraints Guide. [Online]. Available: http://www.xilinx.com/support/documentation/sw_manuals/xilinx11/cgd.pdf

[90] International Workshop on Logic Synthesis, IWLS'93. [Online]. Available: http://www.iwls.org

[91] K. McElvain. (1993) IWLS'93 Benchmark Set: Version 4.0, distributed as part of the IWLS'93 benchmark distribution. [Online]. Available: http://www.cbl.ncsu.edu:16080/benchmarks/LGSynth93/

[92] F. Gray, "Pulse code communication," U.S. Patent 2,632,058, March 1953.

[93] R. W. Hamming, "Error detecting and error correcting codes," Bell System Technical Journal, vol. 29, no. 2, pp. 147-160, 1950.

[94] Xilinx. (2009, January) AR \#23904 - 10.1i MAP_PAR - Will the Xilinx implementation tools give the exact same results with the exact same source files, system, and software version. [Online]. Available: http://www.xilinx.com/support/answers/23904.htm 


\section{Appendix A Glossary}

$3 \mathrm{M}$

Terminology for the number of metal layers available in a CMOS design process. 14, 91

AMI

Semiconductor and Integrated Circuit Devices corporation, formerly AMI Semiconductor. 14,91

ASIC

Integrated Circuit designed using the full-custom design approach for use in a specific application or setting. 91

\section{ASICs}

see ASIC. 1, 91

Asynchronous

Classification of an FSM which operates independently of a clock source and is driven solely on input sequences. 91

Big Endian

Describes a binary string where the left most bit is the MSB and the right most bit is the LSB. 91

\section{BLIF}

Format for describing circuits and systems through text to allow use in design automation tools. 6,91

BSD

Method for converting a bitmap watermarking signature into a directed graph or watermark FSM. vii, 91

\section{CMOS}

A technology for constructing Integrated Circuits. 14, 91

\section{CSFSM}

An FSM which has all possible behavior specified. 8, 9, 91

\section{DEEP SUBM}

Manufacturing feature size for Integrated Circuit technologies. 14, 91 
Appendix A (Continued)

DFA

Class of Finite State Machines in which all sets of input sequences result in reproducible behavior. 9,91

DRC

Process in Integrated Circuit design to ensure that physical design layouts adhere to the rules implemented for the manufacturing process being used. 14, 91

$\mathrm{E}$

Terminology for the availability of a secondary polysilicon layer that can be used for poly capacitors or transistor gates. 14,91

\section{FCFS}

A method for processing elements, specifically, the first elements to arrive are processed first. 49, 68, 91

\section{FPGA}

Reconfigurable computing device to allow for the rapid prototyping and development of computer systems. 2, 91

FSD

Signature Decomposition Technique used on files for creating directed graphs or watermark FSMs. vii, 35, 91

FSM

Abstract modeling method of sequential circuit systems that can be mathematical described using a 6 -tuple. 4, 91

Hard IP

IP in a format which is difficult to alter. 91

HDL

Language, in a human readable format, for designing and specifying the behavior of computer systems. 2, 17, 53, 91

HPC

Typically a collection, or cluster, of computing resources that can be used collectively to achieve a single task. 60,91

HSD

Signature Decomposition Technique used on hash sequences for creating directed graphs or watermark FSMs. vii, 37, 91 
Appendix A (Continued)

$\mathrm{I} / \mathrm{O}$

Describes the interfaces between user and system for communication. 4, 19, 91

IC

System of combinational or sequential logic functions manufactured on a silicon wafer. $1,2,91$

ICSFSM

An FSM which uses don't care conditions or does not define all possible behavior. 8, 10,91

\section{IEEE}

Organization consisting of academics and industry professionals for the advancement of technology. 91

IP

Any material which an individual creates and holds the exclusive rights for. 1, 2, 91

IPC

Reusable hardware design containing logic functions that are IP for a given party. 91 ISE

Interactive software environment that has been integrated into a set of design tools that can otherwise be used through a commandline. 55, 91

\section{IWLS}

Annual conference held for topics relating to synthesis, optimization, and verification of integrated circuits. 67,91

Kiss2

Subset of the BLIF syntax used for describing sequential systems in a modified state table format. 91

Little Endian

Describes a binary string where the right most bit is the MSB and the left most bit is the LSB. 91

LSB

Descriptor used for a position in a binary array based on whether Big or Little Endian principles are used. 21, 22, 91

\section{LUT}

Hardware element used as memory array which returns predefined output conditions based on the inputs to the device. 91 
Appendix A (Continued)

\section{MAX-SAT}

Computationally complex problem which finds maximal group of variable configurations that satisfy the boolean equation in which they are used. 91

\section{Mealy Model}

Modeling for an FSM where the outputs are updated during the transition to the next state. 91

Moore Model

Modeling for an FSM where the outputs are updated after transitioning to the next state. 91

MSB

Descriptor used for a position in a binary array based on whether Big or Little Endian principles are used. 91

$\mathrm{N}$

Terminology for Integrated Circuit Wafer Doping properties. 14, 91 NDFA

Class of Finite State Machines in which all sets of input sequences result in behavior that is unlikely to be reproduced or occur again. 10, 91

Netlist

Hardware device designed at the HDL or transistor level. 91 NP

Describes a set of decision based problems which have verifiable solutions in polynomial time. 91

NP-Complete

Describes a subset of decision based problems which have no polynomial time solutions. $25,41,91$

NP-Hard

Describes a subset of computation problems which have NP-Complete decision versions. 91

PNG

A raster image format that uses lossless data compression techniques. 37, 91

RIPEMD-160

160-bit cryptographic hash function. 37, 91 
Appendix A (Continued)

SC

A method for CMOS fabrication processes that provide design abilities nearly independent of process and metric. 14, 91

\section{SCN3M}

Terminology used for describing the AMI C5F CMOS Process, Scalable CMOS, N substrate, 3 Metals. 14, 91

\section{SCN3ME}

Terminology used for describing the AMI C5N CMOS Process, Scalable CMOS, N substrate, 3 Metals, Electrode. 14, 91

\section{SHA-1}

160-bit bit cryptographic hash function. 60, 91

SOC

Integrated circuit which includes all necessary components for an electronic system on a single chip. 91

SOCs

See SOC. 91

Soft IP

IP in a format which can be easily altered. 91

SRAM

Type of volatile memory that uses cross-coupled inverters to maintain the internal value and removes the need for this value to be refreshed. 91

State Encoding

An arbitrary or intentionally assigned binary or text string used for labeling states in an FSM. 91

STG

Graphical method for representing FSMs as a collection of nodes and edges. 5, 91

$\mathrm{STT}$

Tabular, non-graphical, method for representing an FSM. 6, 91 SUBM

Manufacturing feature for Integrated Circuit technologies.. 14, 91

Synchronous

Classification on an FSM which operates on the rising or falling edge of a clock signal. 91 
Appendix A (Continued)

VHDL

Programming language for FPGA devices. 53, 91

VHDL-93

The IEEE Standard 1076, Revision 1993, version of VHDL. 53, 91

VHSIC

United States Department of Defense project which led to the development of VHDL. 53,91

Watermark

A file, video, audio, or text message used as a unique signature hidden in IP. 91

$\mathrm{XST}$

A tool in the Xilinx ISE Design Suite used for the synthesis and optimization of HDL designs. 53, 55, 91 


\section{Appendix B Permission of Use}

(C)2012 IEEE. Reprinted, with permission, from M. Lewandowski, R. Meana, M. Morrison, S. Katkoori, "A Novel Method for Watermarking Sequential Circuits," 2012 IEEE International Symposium on Hardware-Oriented Security and Trust, June 2012.

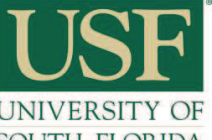

SOUTH FLORIDA

March 20, 2013

Matthew Lewandowski,

Graduate Student,

Computer Science and Engineering,

University of South Florida,

NarMOS Research Team

Dear Matthew,

You have permission to use the USF College of Engineering co-branded USF logo as an example of the capabilities of the HASH-2-K2 method detailed in your Master's Thesis. This permission is specific to use of the logo for this purpose and does not include permission to mark any product for sale.

Thank you,

Anne Scott

Senior Graphic Designer

University Communications \& Marketing

University of South Florida

4202 E Fowler Ave, STOP CGS 301

Tampa, FL 33620-4301

813-974-9043

fax 813-974-2888

UNIVERSITY COMMUNICATIONS \& MARKETING

University of South Florida • 4202 East Fowler Avenue, CGS $301 ・$ Tampa, FL 33620-4301

813) $974-4014 \bullet$ FAX (813) $974-2888 \bullet$ www.usf.edu

This letter is an electronic communication from the University of South Florida. 


\begin{abstract}
About The Author
Matthew Lewandowski will be receiving both his Bachelor's and Master's Degree in Computer Engineering Spring 2013 from the University of South Florida in Tampa, FL. Upon graduation, he will be continuing on in academia and to pursue a Doctorate Degree Fall 2013 with USF. His goal is to become a professor in the area of CMOS-VLSI Design and has assisted Dr. Srinivas Katkoori in teaching the course at USF over the past two years. His research interests are Design of Secure Hardware, Design Automation, and Reversible Logic. He is a recipient of the Design Automation Conference Young Student Scholarship Program, and has been awarded a Best Post Award for his research at both USF's College of Engineering Research Day, and $9^{\text {th }}$ Annual Undergraduate Research Symposium. Recently, with his research team dubbed "NarMOS," he was awarded First Place at NYU-Poly's CyberSecurity Awareness Week Embedded System Challenge. He considers his personal achievements to be his continued success in the development of a Cadence Design Kit and ON-Semiconductor AMI_C5N Process Design Kit which are being used with the CMOSVLSI Design, Design Automation, and Digital Design in Nano-Scaled Technologies courses at USF.
\end{abstract}

